NBER WORKING PAPER SERIES

\title{
GLOBALIZATION, RETURNS TO ACCUMULATIONS AND THE WORLD DISTRIBUTION OF OUTPUT
}

\author{
Paul Beaudry \\ Fabrice Collard \\ Working Paper 10565 \\ http://www.nber.org/papers/w10565

\section{NATIONAL BUREAU OF ECONOMIC RESEARCH 1050 Massachusetts Avenue Cambridge, MA 02138} \\ June 2004
}

The authors would like to thank Daron Acemoglu, Ricardo Caballero, Martial Dupaigne, Patrick Francois, David Green, Ashok Kotwal, Marco Ma®ezoli, Fabrizzio Zilibotti, three anonymous referees and seminar participants at Delta\{Paris, MIT, Warwick University, University of Montreal for very helpful discussions. The views expressed herein are those of the author(s) and not necessarily those of the National Bureau of Economic Research.

(C)2004 by Paul Beaudry and Fabrice Collard. All rights reserved. Short sections of text, not to exceed two paragraphs, may be quoted without explicit permission provided that full credit, including (C) notice, is given to the source. 
Globalization, Returns to Accumulations and the World Distribution of Output Paul Beaudry and Fabrice Collard

NBER Working Paper No. 10565

June 2004

JEL No. O33, O41

\title{
$\underline{\text { ABSTRACT }}$
}

This paper examines the extent to which the process of globalization can explain the observed widening in the cross--country distribution of output--per--worker. In particular, we examine whether the opening up of trade in a Hecksher--Ohlin type model of trade can explain the observed changes. On the theoretical front the model highlights that, when the labor market is subject to a holdup problem, then the opening up of trade can cause an increase in the dispersion of income across countries similar to that observed in the data due to the emergence of a discrepancy between the private and social returns to capital accumulation that favors capital abundant countries. On the empirical front, we document the relevance of the model by examining whether growth patterns, decomposition exercises and specialization patterns support the model's predictions. Overall we find that over $50 \%$ of the recently observed increase in income dispersion across countries can be accounted for by the mechanism exemplified by the model.

\author{
Paul Beaudry \\ Department of Economics \\ University of British Columbia \\ 997-1873 East Mall \\ Vancouver, BC \\ Canada, V6T 1Z1 \\ paulbe@interchange.ubc.ca \\ Fabrice Collard \\ University of Toulouse \\ CNRS-GREMAQ and IDEI \\ Manufacture des tabacs, bat. F \\ 21 allee de Brienne \\ 310000 Toulouse
}




\section{Introduction}

Over the last few decades, the volume of international trade has increased enormously, vastly outpacing the growth in world GDP. This observation is often regarded as a defining element of the process of globalization. Interestingly, over the same period, the distribution of income across countries has changed considerably. For example, over the period 1960-1998, the distribution of output-per-worker across countries hollowed-out substantially, as mass moved away from the mean of the distribution towards two emerging modes (see Quah [1997], Jones [1997], Beaudry, Collard and Green [2002] among others), thereby giving rise to (i) greater dispersion in income across countries and (ii) the appearance of a bi-modal or Twin-Peaked distribution. This simultaneity among the two phenomena is rather intriguing and it is natural to ask whether they may be related. In particular, it is relevant to ask whether the different growth performances underlying the change in the world distribution may be the result of an unequal distribution of the gains associated with globalization.

In this paper, we explore whether basic trade theory may offer an empirically relevant explanation to the observed change in the cross-country distribution of income. The main claim of the paper is that a dynamic version of the Hecksher-Ohlin model of trade, once extended to allow for an imperfection in the labor market, provides a simple explanation for why the opening up of trade may be associated with increased dispersion in income across countries and the possible emergence of bi-modality. Moreover, and especially important, we show that the central mechanism driving the result receives considerable support in the data. Although the model is standard on many fronts, it departs from much of the literature by introducing a hold-up problem in the labor market which allows workers to extract rents from firms with high capital intensity.

The benchmark model we develop is one with one final good and two intermediate goods. The process of globalization is modelled as the opening up of trade in intermediate goods. The source of comparative advantage in the model is due to differences in capital abundance driven by cross-country differences in savings rates and rates of population growth. We contrast the behavior of the model for both the case with and without the presence of a holdup problem in the labor market. In the absence of any labor market imperfections, we show why the opening up of trade is unlikely to have a substantial impact on the cross-country distribution

of income. However, once the possibility of rent extraction in the labor market is allowed, we show that the opening up of trade in intermediate goods will generate increased dispersion in income across countries and can generate the emergence of a bi-modal distribution. One of the key elements of the model is the interaction between the location of rent paying firms and the process of capital accumulation. In particular, the main determinant of whether a country gains 
disproportionately from the opening up of trade in our model depends on its tendency to be capital abundant. For example, a country with a high saving rate and a low rate of labor force growth will tend to be capital abundant and will therefore tend to specialize in the production of high-productivity high-capital-intensive goods during the process of globalization. This aspect of the model leads to the main implication that, during the process of globalization, one should witness the emergence of an abnormally strong link between a country's growth rate and its tendency to favor capital deepening. As we shall show, this and other predictions of the model find considerable support in the data.

It is immediately worth emphasizing that the mechanism driving increased dispersion in our model is distinct from the one implied in Ventura [1997]. In a model such as Ventura's, globalization will affect the distribution of income across countries mainly by changing the distribution of capital-output ratios. In particular, countries with low discount factors take advantage of the absence of decreasing returns to capital to increase their savings rate and thereby their capital-output ratios. In contrast, in our model, globalization increases the social returns to being capital abundant, thereby causing increased dispersion even in the absence of any change in capital-output ratios across countries. In other words, the differences between these two approaches for explaining an increase in dispersion reduces to the distinction between a change in the returns to capital accumulation versus a change in the distribution of capital-output ratios across countries. Since such mechanisms are fundamentally different but possibly complementary, in the empirical section of the paper we will evaluate the relative importance of each mechanism.

The remaining sections of the paper are as follows. In section 1, we review a set of observations related to changes in the world distribution over the period 1960-98 (these draw on observations documented in Beaudry et al. [2002], hereafter BCG) and we present a simple conceptual framework for analyzing these observations. In section 2, we present the benchmark model. Section 3 analyses the main implications of the model, with emphasis on its predictions in terms of distribution of output-per-worker. In Section 4, we examine the main empirical implications of the model. Since many of the assumption in the benchmark model are rather extreme, in the appendix we discuss generalizations. Finally, the last section offers concluding remarks.

\section{Changes in the Distribution of Output-per-Worker}

In this section, we first review the salient changes in the cross-country distribution of the outputper-worker that occurred over the period 1960-1998. ${ }^{1}$ Then we present a simple framework

\footnotetext{
${ }^{1}$ Many of the observations discussed in this section can also be found in other work such as Quah [1993] Jones [1997], and Beaudry et al. [2002].
} 
which helps clarify the approach and that we will adopt to analyze this phenomenon.

\subsection{Empirical Evidence}

The main changes in the cross-country distribution of (log) output per worker are summarized in Figure 1. The figure reports the distribution of $(\log )$ output-per-worker for the three years 1960, 1978 and 1998. The data are taken from the World Penn tables 6.0. ${ }^{2}$ We choose to highlight here the change in distribution for the set of Non-Sub-Saharan African countries as to emphasize changes which are not simply the results of the well-known poor growth performance of the Sub-Saharan Africa countries. ${ }^{3}$

The plotted distributions are kernel density estimates. ${ }^{4}$ Each of the three distributions is expressed as deviations from the US (log) level of output-per-worker in the corresponding year in order to emphasize changes in the shape of the distribution. It should however be noted that the actual distribution shifted substantially to the right from 1960 to 1998. The average outputper-worker increased by $134 \%$ between 1960 and 1998 for the 75 countries we consider, implying an average annualized rate of growth of $2.27 \%$. As can be seen from the figure, the shape of

Figure 1: Across-Country (log-)Income Distribution: 1960-1998

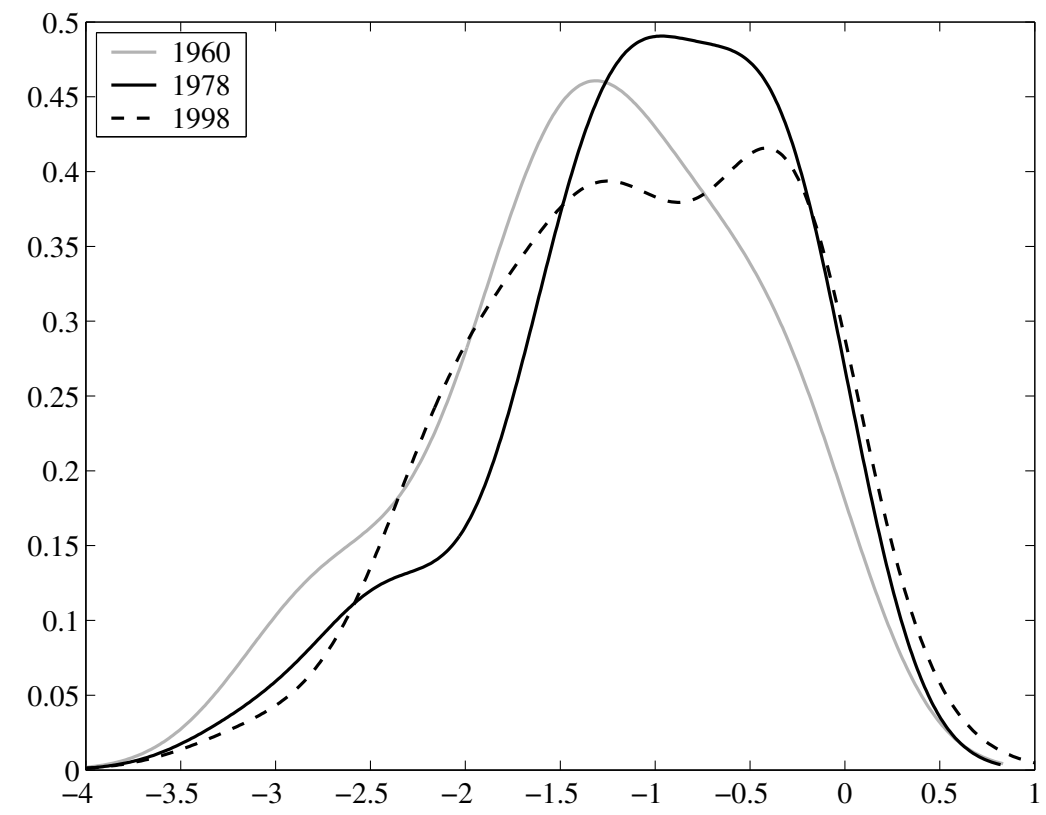

the distribution changed considerably from 1960 to 1998. In 1960, it was clearly uni-modal

\footnotetext{
${ }^{2}$ See Appendix $\mathrm{C}$ for the list of countries.

${ }^{3}$ To see the corresponding changes in the distribution that include the Sub-Saharan countries, see Beaudry et al. [2002].

${ }^{4}$ The density estimates are computed using the Rosenblatt-Parzen kernel density estimator. We used a Gaussian kernel, with an optimal bandwidth parameter chosen as $h=1.0592 \sigma N^{-1 / 5}$ where $\sigma$ is the standard deviation of the data and $N$ is the number of observations.
} 
and close to a normal distribution. In contrast, by 1998, the distribution appears somewhat bimodal. This observation corresponds to what Quah [1993] and Jones [1997] call the twin-peaks phenomenon. ${ }^{5}$

Another important element to note in Figure 1 is the differential movement in the distribution over the period 1960-78 versus 1978-98. Over the first sub-period, 1960 to 1978, the distribution was actually shifting slightly to the right, suggesting that most countries were slowly catching up with the US. This movement is totally in lines with the convergence phenomenon predicted by standard neoclassical growth theory. However, over the period 1978 to 1998, this process entirely reversed itself as distribution widened considerably and the density in the middle of the distribution decreased substantially. In effect, it is in this second period (1978-98) that the twin-peaks phenomenon emerged.

In order to get a better sense of the timing of the change in dispersion in output-per-worker across countries, Figure 2 reports time series plots for three percentile differences: the 30-70 differences, the 25-75 difference and the 20-80 differences. ${ }^{6}$ We begin by focusing on these percentile differences since Figure 1 indicates that the widening in the distribution of the period 1978-98 is not governed by movements in the tails of the distribution but more akin to movements in the interquartile range. In effect, throughout the paper we will often refer to a widening of a distribution when there are increases around the inter-quartile range even if there is no widening in the tails.

As can be seen from the figure, these three percentile differences were rather stable through most of the sixties and up to the end of the 1970s. Then, starting somewhere around 1978, the dispersion — as measured by these percentile differences — started increasing substantially. In particular, the interquartile range increased by about $40 \%$ over the period, after being quite stable up to 1978. Table 1 reports the numerical values of a large set of percentile differences as to further emphasize that the increased dispersion observed over the period 1960-98 arose almost entirely within the period 1978 to 1998. For instance, let us again consider the three percentile differences, 20-80, 25-75 and 30-70. Over the period running from 1960 to 1978, these three percentile differences decreased illustrating a convergence phenomenon across countries. Also note that these changes are of about -0.1 for the two first differences and -0.02 for the last one. The pattern changed dramatically over the period 1978-98, as the differences increased over the period for all three percentiles. Not only did this undo the changes over the previous period, it actually generated increased dispersion for the two periods combined.

\footnotetext{
${ }^{5}$ Kremer, Onatski and Stock [2001] question the appropriateness of referring to this distribution a being twin peak.

${ }^{6}$ The percentile differences are directly calculated from the data and not from the kernel estimates of the density.
} 
Figure 2: Percentile differences

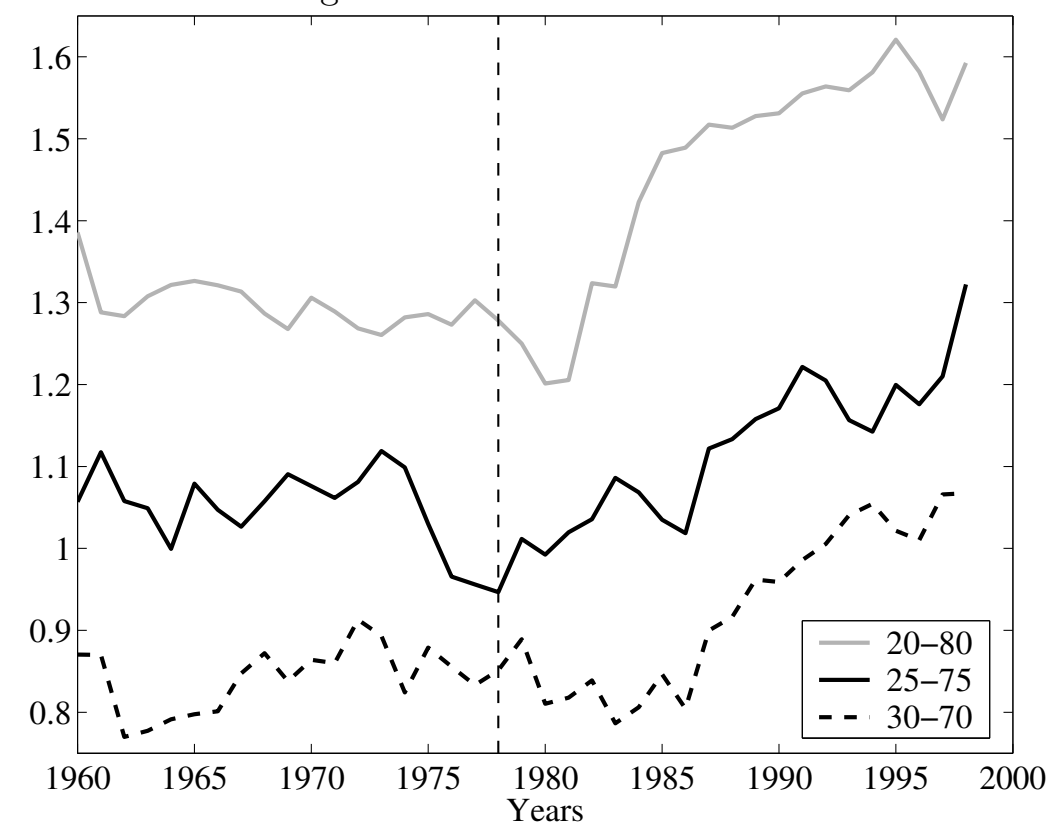

Table 1: Changes in inter-quantile ranges: Output-per-worker (All countries)

\begin{tabular}{ccccccccc}
\hline \hline & \multicolumn{3}{c}{ Ranges } & & \multicolumn{3}{c}{ Changes } \\
\cline { 2 - 3 } \cline { 6 - 8 } & 1960 & 1978 & 1998 & & $60-78$ & $78-98$ & $60-98$ \\
\hline $10-90$ & 2.270 & 2.067 & 1.905 & & -0.203 & -0.162 & -0.365 \\
$15-85$ & 1.762 & 1.396 & 1.840 & & -0.366 & 0.444 & 0.078 \\
$20-80$ & 1.386 & 1.278 & 1.593 & & -0.108 & 0.314 & 0.207 \\
$25-75$ & 1.057 & 0.947 & 1.322 & & -0.110 & 0.376 & 0.265 \\
$30-70$ & 0.870 & 0.851 & 1.067 & & -0.019 & 0.216 & 0.197 \\
$35-65$ & 0.604 & 0.597 & 0.820 & & -0.008 & 0.224 & 0.216 \\
$40-60$ & 0.338 & 0.384 & 0.486 & & 0.046 & 0.103 & 0.148 \\
\hline \hline
\end{tabular}


In Table 2, we report the same set of percentile differences but we now split the sample into two sub-groups. The two sub-groups are differentiated according to their ratio of international trade (sum of imports and exports) to GDP, which is a common measure of openness. In particular,

Table 2: Changes in inter-quantile ranges: Output-per-worker (Split sample)

\begin{tabular}{|c|c|c|c|c|c|c|}
\hline & \multicolumn{3}{|c|}{ Ranges } & \multicolumn{3}{|c|}{ Changes } \\
\hline & 1960 & 1978 & 1998 & $60-78$ & $78-98$ & $60-98$ \\
\hline \multicolumn{7}{|c|}{ (a) Openness $<$ median } \\
\hline $10-90$ & 2.220 & 2.395 & 2.010 & 0.175 & -0.385 & -0.210 \\
\hline & 2.069 & 2.003 & 1.737 & -0.066 & -0.267 & -0.333 \\
\hline $20-80$ & 1.579 & 1.629 & 1.348 & 0.050 & -0.281 & -0.231 \\
\hline $25-$ & 1.094 & 0.997 & 1.029 & -0.097 & 0.031 & -0.066 \\
\hline 30 & 0.766 & 0.712 & 0.724 & -0.054 & 0.013 & -0.041 \\
\hline $35-$ & 0.480 & 0.479 & 0.536 & -0.000 & 0.057 & 0.057 \\
\hline $40-60$ & 0.372 & 0.271 & 0.372 & -0.101 & 0.101 & 0.000 \\
\hline \multicolumn{7}{|c|}{ (b) Openness $\geqslant$ median } \\
\hline $10-$ & 1.785 & 1.528 & 2.000 & -0.257 & 0.472 & 0.215 \\
\hline $15-85$ & 1.409 & 1.336 & 1.830 & -0.073 & 0.494 & 0.422 \\
\hline $20-80$ & 1.225 & 1.120 & 1.570 & -0.105 & 0.450 & 0.345 \\
\hline $25-75$ & 1.084 & 0.924 & 1.127 & -0.161 & 0.203 & 0.042 \\
\hline $30-70$ & 0.738 & 0.741 & 1.003 & 0.004 & 0.262 & 0.266 \\
\hline $35-65$ & 0.548 & 0.542 & 0.823 & -0.007 & 0.282 & 0.275 \\
\hline $40-60$ & 0.305 & 0.418 & 0.636 & 0.113 & 0.218 & 0.331 \\
\hline
\end{tabular}

the first sample corresponds to the set of countries with a below median level of trade-to-GDP ratio in the period $1960-78 .{ }^{7}$ The movement in percentile differences for this sample is given in Panel (a) of the table, while Panel (b) reports the changes in percentile differences for the sample with an above median trade-to-GDP ratio. The main elements we want to emphasize in Table 2 is that the increased dispersion observed over the period 1978-98 in the whole sample appears to be a characteristic of the more open economies but not of the less open economies. In fact, for the more open economies, we see that all the percentile differences increased quite substantially over the period 1978-98. For instance, the 25-75 percentile difference increased by 0.203 over this period in the more open economies, but remained almost steady (0.031) in the less open economies. This phenomenon is even more pronounced for the 20-80 percentile difference, as it changed by 0.450 over this period in more open economies, while the less open economies continued to experience a decrease in this difference. In contrast, over the period 1960-78, the percentile differences in both groups moved similarly and tended to be quite stable. Last, let us note that the average growth in these two subs-groups was very similar. Indeed, the more open economies experimented an average annualized rate of growth of $3.22 \%$ per year

\footnotetext{
${ }^{7}$ The results are almost identical if we divide the sample using the average trade-to-GDP ratio over the entire period as opposed to using the $1960-78$ period.
} 
over the period $1960-1978$ and $1.32 \%$ for the period $1978-1998$. The corresponding average annualized rate of growth in the less open economies are respectively $3.31 \%$ and $1.37 \%$.

The above set of observations leads us to ask whether the observed change in the distribution over the period 1978-98 versus the period 1960-78 could be related to an increase in new trade opportunities available to open countries which subsequently lead to an explosion in the volume of world trade. ${ }^{8}$ In the following section we present a simple model of globalization which addresses this question. But before moving to the theoretical model, it is helpful to outline a general framework for thinking about how globalization can affect the determination of income and thereby its distribution. In particular, this framework allows to highlight the approach we will adopt in the remaining sections of the paper.

\subsection{A Simple Framework}

When considering how the process of globalization may affect the cross-country distribution of income, it is useful to breakdown the effects into two distinct channels, as illustrated in the next diagram.

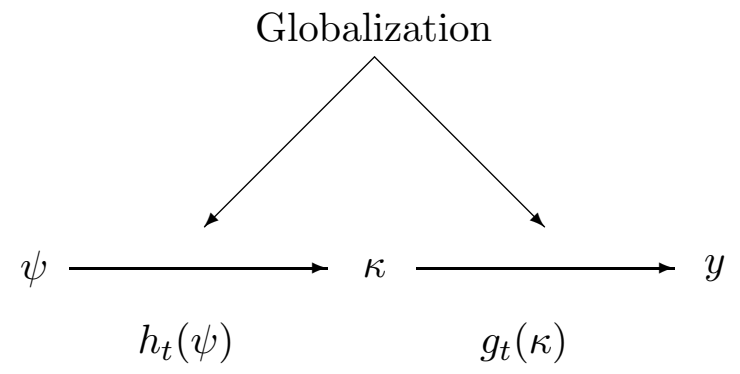

On the one hand, let there be a set of exogenous country-specific driving forces, denoted by $\psi$, which affects a country's accumulation patterns in terms of investment rate and capital intensity, which we summarize by $\kappa$. These exogenous driving forces, $\psi$, may be preferences, policies and possibly demographics. Let the mapping between $\psi$ and $\kappa$ be represented by the function $h_{t}(\cdot)$. Given accumulation patterns, then the agents in the economy decide how best to use their resources to determine output-per-worker, denoted by $y .{ }^{9}$ We will denote this second mapping from accumulation patterns to output-per-worker by the function $g_{t}(\cdot)$. We have indexed the two mappings by time to highlight that they may change over time. In fact, as a framework,

\footnotetext{
${ }^{8}$ From 1960 to the early 1970s (1972), world openness grew, on average, by only 0.19 point per year. The growth in world trade then started to accelerate as the average growth was 0.69 points per year between 1972 and 1998, with the growth rate between 1986 and 1998 being even faster as to reach a level of 0.90 point per year. In total, the degree of world openness as measured by the increase in the ratio of trade-to-gdp grew from $23.72 \%$ in 1960 to $44.08 \%$ in 1998.

${ }^{9}$ Note that in this framework, globalization is only allowed to have a level effect of $Y$. Accordingly, throughout this paper we will explore a mechanism that has a level effect and no growth effect.
} 
we want to consider the process of globalization as potentially changing both the form of the mapping from $\psi$ to $\kappa$ and the form of the mapping from $\kappa$ to $y$.

In order to understand the relationship between changes in the functions $h_{t}(\cdot)$ and $g_{t}(\cdot)$ and the changes in the distribution of $y$, it is helpful to make use of the standard change of variable formula to derive the pdf of $y_{t}$, denoted $\mu_{t}^{\mathrm{Y}}($.$) . In this case, the pdf of y$ is a function of the distribution of $\psi$, denoted $\mu_{t}^{\psi}($.$) , and the functions h_{t}(\cdot), g_{t}(\cdot)$. This relationship is given below, where we evaluate relationship at the median value of $y$ to allow the clearest expression:

$$
\mu_{t}^{\mathrm{Y}}\left(y^{\mathrm{M}}\right)=\frac{\mu_{t}^{\psi}\left[\psi^{\mathrm{M}}\right]}{\left|g_{t}{ }^{\prime}\left(h_{t}\left(\psi^{\mathrm{M}}\right)\right) h_{t}{ }^{\prime}\left(Z^{\mathrm{M}}\right)\right|}
$$

where $\psi^{\mathrm{M}}$ is the median value of $\psi$. The important aspect to note from the above expression is how the density of $y$ relates to the slopes of the functions $h_{t}(\cdot)$ and $g_{t}(\cdot)$. Indeed, if the distribution of $\psi$ does not change over time, then the mass near the middle of the distribution of $y$ will decrease if either the slope of $h_{t}(\cdot)$ or $g_{t}(\cdot)$ increases. In other words, a potential explanation to the observed hollowing out of the distribution of $y$ is that either the slope of $h_{t}(\cdot)$ or $g_{t}(\cdot)$ increased over time. The explanation we present will focus on an increase in the slope of $g_{t}(\cdot)$, that is, an increase in the sensitivity of $y$ to capital accumulation.

In this paper, we will undertake three steps in order to evaluate whether the recent period of globalization may have contributed to the observed changes in the cross-country distribution of income. First, we will highlight the predictions of trade theory regarding how the opening up of trade affects the mapping from a country's accumulation patterns to the determination of $y$, that is, we will illustrate how globalization may affect the function $g_{t}(\cdot)$ and thereby affect the distribution of $y$. Second we will evaluate empirically whether there has been a change over time in the mapping between a country's accumulation patterns and the determination of output-per-worker, and we will examine whether such a change is consistent with that implied by the theory. Third, we will examine how much of the change in the distribution of $y$ over the period 1978-98 may be accounted for by the change in the mapping $g_{t}(\cdot)$, that is, we will gauge the empirical relevance of this one channel in affecting the distribution of $y$. In addition, we will compare the relative importance of the change in channel $g_{t}(\cdot)$ versus $h_{t}(\cdot)$ in explaining the change in the distribution of $y$. This last step is particularly relevant since, as we will make clear, in the type of model presented in Ventura [1997], globalization affects the determination of $y$ largely through its effects on $h_{t}(\cdot)$. The main claim of this paper is that the change in the mapping $g_{t}(\cdot)$ is central to understand the recent changes in the distribution of $y$.

Since in the theory section, we want to highlight how the opening changes the mapping between accumulation patterns and output-per-worker, we will adopt an approach that treats accumulation patterns as exogenous. To this end we will follow Solow [1956] and have capital accumulation being governed by an exogenous country specific investment rate and a country 
specific rate of labor force growth. This simplification will allow us to clearly focus on how the opening of trade may affect the mapping $g_{t}(\cdot)$. However, in the empirical section we will recognize that investment rates are not exogenous and we will use several different instrumental variable strategies to evaluate whether the mapping $g_{t}(\cdot)$ changed between the periods 1960-78 and 1978-98 in a manner consistent with the theory. The previous framework emphasizes particular links that may be relevant for understanding the observed change in the distribution of $y$. However, it is worth noting that these links are not the only possible types of explanation. In particular, it is quite natural to first think that a widening of a distribution may be primarily driven by a change in the dependence of growth outcomes on initial condition. Indeed, if in the first period (1960-78), growth was negatively related to a country's initial level, while in the second period the relationship was positive, then such a change of dependance on initial conditions could easily explain the type of change observed in Figure 1. Although this is not the channel that will be implied by our model, in the empirical section we will nonetheless evaluate its merit relative to channel emphasized in the model.

\section{The model}

In this section we present a model of the world economy consisting of a set of countries that all produce the same final good, which can be either consumed or invested. The final good is produced by means of two intermediate goods. Our objective with this model is to illustrate how the opening of new trading opportunities affects the cross-country distribution of income. Most of the analysis will be conducted in the absence of international financial markets, although in the appendix we discuss how our results extend to the case with international capital flows. An important element of our analysis will be to contrast how effects of trade differ depending on whether the labor market functions in a frictionless manner or whether it is subject to an imperfection that results in more capital intensive firms paying higher wages.

\subsection{Individual behaviors}

Let the world be composed of a collection of $\mathrm{N}$ countries indexed by $i, i=0,1, \ldots, N$, where country 0 will be used as a reference country. In each country there is only one non-tradable final good, $Y_{i, t}$, which is produced using two tradable intermediate goods $Z_{1, i, t}$ and $Z_{2, i, t}$ according to the following production function:

$$
Y_{i, t}=\left(Z_{1, i, t}\right)^{\varphi}\left(Z_{2, i, t}\right)^{1-\varphi} \text { with } \varphi \in(0,1)
$$

Initially, there will be no trade in the intermediate goods, and then, due to what may be referred to as the process of globalization, we will allow for trade in these goods where it is the production 
of each intermediate goods net of exports which is used in the production of the final good. The objective in this section is to illustrate why such a process may change the determination of output-per-worker and thereby change its dispersion.

The intermediate goods $Z_{1}$ and $Z_{2}$ can be produced domestically using physical capital, $K$, and labor, $L$, according to constant returns-to-scale technologies represented by the production functions:

$$
\begin{aligned}
& Z_{1, i, t}=\Theta_{1} K_{1, i, t}^{\alpha}\left(\Omega_{i} \Gamma_{t} L_{1, i, t}\right)^{1-\alpha} \text { with } 0 \leqslant \alpha \leqslant 1 \\
& Z_{2, i, t}=\Theta_{2} K_{2, i, t}^{\beta}\left(\Omega_{i} \Gamma_{t} L_{2, i, t}\right)^{1-\beta} \text { with } 0 \leqslant \alpha \leqslant \beta \leqslant 1
\end{aligned}
$$

where $\Theta_{1}$ and $\Theta_{2}$ are sector specific constants and where $\Omega_{i} \Gamma_{t}$ is the country specific level of labor augmenting knowledge at time $t$. Note that this formulation allows for a country specific component in labor augmenting knowledge, $\Omega_{i}$, and allows for a common time varying component $\Gamma_{t}$ which is assumed to grow at the exogenous rate $\gamma \geq 0$, that is, $\Gamma_{t+1}=(1+\gamma) \Gamma_{t}, \Gamma_{0}>0$ given. The most important aspect that differentiates the two intermediate goods is that $\beta \geqslant \alpha$, which means that the intermediate good $Z_{1}$ is less capital intensive than $Z_{2}$.

We will assume that all producers of the intermediate goods and the final goods maximize profits taking prices, wages and the rental rate on capital as given. This yields the following standard input demand functions for capital and labor in each sector:

$$
\begin{aligned}
& \left.\begin{array}{rl}
\alpha P_{1, i, t} Z_{1, i, t} & =q_{i, t} K_{1, i, t} \\
\alpha) P_{1, i, t} Z_{1, i, t} & =W_{1, i, t} L_{1, i, t}
\end{array}\right\} \quad \text { in sector } 1 \\
& \left.\begin{array}{rl}
\beta P_{2, i, t} Z_{2, i, t} & =q_{i, t} K_{2, i, t} \\
\beta) P_{2, i, t} Z_{2, i, t} & =W_{2, i, t} L_{2, i, t}
\end{array}\right\} \quad \text { in sector } 2
\end{aligned}
$$

where the price of the final good is normalized to 1 , and where $P_{1, i, t}, P_{2, i, t}, q_{i, t}, W_{1, i, t}$ and $W_{2, i, t}$ denote respectively, for economy $i$ at time $t$, the price of the intermediate goods, the rental price of capital and the wage rates in sector 1 and in sector 2 . In the absence of trade in intermediate goods, the prices $P_{1}$ and $P_{2}$ will adjust to equal domestic supply and demand for goods $Z_{1}$ and $Z_{2}$. In the presence of trade in intermediates, the prices $P_{1}$ and $P_{2}$ will be set on the international market, and $Z_{1}, Z_{2}$ will represent the sum of imported plus domestically produced good.

In contrast to the market for goods which is assumed to behave in a Walrasian fashion, we want to allow for the possibility of non-Walrasian wage determination. In particular, we will assume that workers can extract rents from firms due to the quasi-fixed nature of capital, that is, we introduce the possibility of a hold-up problem between a firm and its workers. As is well known, there are many ways to model such a possibility. One quite general implication of many such models is that wages are higher in the more capital intensive firms. ${ }^{10}$ In order to capture this

\footnotetext{
${ }^{10}$ There is a large empirical literature which supports the notion that high capital intensive firms or high
} 
idea in its simplest form, we will assume that wages in each sector are determined by a monopoly union which takes labor demand as given and maximizes the following objective function:

$$
\left(W_{j, i, t}-W_{j, i, t}^{R}\right) L_{j, i, t}^{\theta} \text { with } 1 \leqslant \theta, \quad j=1,2
$$

where $W_{j, i, t}^{R}$ is the reservation value of time for workers in sector $j$ in economy $i$ at period $t$. Furthermore, we will assume that labor is supplied inelastically and hence the flow value of being unemployed is zero. In this case, it is easy to verify that the unique equilibrium wage setting outcome corresponds to (i) wage equating demand and supply for jobs associated with producing the good $Z_{1}$, that is, in the less capital intensive sector there is no rent extraction, and (ii) wages for jobs associated with producing $Z_{2}$ are set as a markup of $x \%$ over jobs in sector $1 .^{11}$ This implies the following relationship between wages in the two sectors.

$$
W_{2, i, t}=\frac{\theta}{\theta-\beta} W_{1, i, t}=(1+x) W_{1, i, t}
$$

Note that as $\theta$ tends to infinity, the wage premium in sector 2 - denoted by $x$ - tends to zero and hence wages are determined in a fully competitive manner. In what follows, we will want to contrast how the process of globalization affects the cross-country distribution of income when $x$ equals zero versus when $x>0$.

In order to complete the description of this economy, it is necessary to specify the dynamics of labor and physical capital. Following traditional growth theory (ex: Solow [1956] and Mankiw, Romer and Weil [1992]), we will assume that labor is supplied inelastically and grows at the exogenous rate $n_{i} \geqslant 0$ such that

$$
L_{i, t+1}=\left(1+n_{i}\right) L_{i, t}, L_{i, 0} \text { given }
$$

And we also assume that investment is determined by an exogenous savings rate $s_{i} \in(0,1)$ such that the law of motion of capital is given by

$$
K_{i, t+1}=s_{i} Y_{i, t}+(1-\delta) K_{i, t}, K_{i, 0}>0 \text { given }
$$

Note that in this formulation, we are assuming away any international capital flows. In the appendix we discuss how the relaxation of this assumption affects our results. We will also later discuss how the analysis would be affected by allowing an optimizing household to determine the saving rate as for example is done in Ventura [1997].

capital intensive industries generally pay wage premia. For example, see Dickens and Katz [1987], Gibbons and Katz [1992], Krueger and Summers [1988] for evidence in support of the notion that employment in high capital intensive industries is associated with a wage premia. The more recent literature using matched worker-firm data also finds that wage premia are generally associated with high capital intensive firms (see e.g. Abowd, Kramarz and Margolis [1999] and Arai [2003]).

${ }^{11}$ If the flow value of being unemployed was sufficiently high, then the presence of monopoly unions would generate equilibrium unemployment. We are ruling out this case by assumption. 


\subsection{Equilibrium}

We are interested in comparing two equilibrium outcomes for this set of economies: the outcome in the absence of trade in $Z_{1}$ and $Z_{2}$, and the outcome in its presence.

In either case, a world equilibrium consists of a sequence of prices $\mathrm{P}=\left\{W_{j, i, t}, q_{i, t}, P_{j, i, t} ; j=\right.$ $1,2 ; i=1, \ldots, N\}_{t=0}^{\infty}$, and a set of allocations $\mathrm{Q}=\left\{Y_{i, t}, K_{j, i, t}, L_{j, i, t}, Z_{j, i, t} ; j=1,2 ; i=1, \ldots, N\right\}_{t=0}^{\infty}$ such that,

1. Given the sequence of prices P, Q solves the firms' problem;

2. Capital accumulates according to equation (7);

3. Given a sequence of allocations, Q, P clears the markets in the sense

$$
\begin{aligned}
L_{i, t} & =L_{1, i, t}+L_{2, i, t} \\
K_{i, t} & =K_{1, i, t}+K_{2, i, t} \\
Y_{i, t} & =\left(Z_{1, i, t}\right)^{\varphi}\left(Z_{2, i, t}\right)^{1-\varphi}
\end{aligned}
$$

4. International markets clear in the sense that

- In the absence of trade (autarky):

$$
Z_{1, i, t}=\Theta_{1} K_{1, i, t}^{\alpha}\left(\Omega_{i} \Gamma_{t} L_{1, i, t}\right)^{1-\alpha} \text { and } Z_{2, i, t}=\Theta_{2} K_{2, i, t}^{\beta}\left(\Omega_{i} \Gamma_{t} L_{1, i, t}\right)^{1-\beta}
$$

- If there is free trade, $P_{j, i, t}, j=1,2$ is independent of $i\left(P_{j, i, t}=P_{j, t}\right)$ and trade is balanced in each economy:

$$
P_{1, t}\left(Z_{1, i, t}-\Theta_{1} K_{1, i, t}^{\alpha}\left(\Omega_{i} \Gamma_{t} L_{1, i, t}\right)^{1-\alpha}\right)+P_{2, t}\left(Z_{2, i, t}-\Theta_{2} K_{2, i, t}^{\beta}\left(\Omega_{i} \Gamma_{t} L_{1, i, t}\right)^{1-\beta}\right)=0
$$

In the above model, countries are allowed to differ along two dimensions: (i) they can have different levels of labor augmenting knowledge $\Omega_{i}$, and (ii) they can have different propensities to favor capital deepening as given by the index $\nu_{i}=\frac{s_{i}}{(1+n)(1+\gamma)-(1-\delta)}$. Note that the index $\nu_{i}$ corresponds to a countries steady state capital-output ratio and therefore a country with a high value of $\nu$ will be more capital abundant in the steady state. Since we want to highlight how globalization will change the role played by the distribution of $\nu_{i}$ in shaping the distribution of output-per-worker, in what follows, notation will be considerably reduced by temporarily abstracting from difference across countries in labor augmenting knowledge, that is we will temporarily set $\Omega_{i}=1$. An attractive feature of this simplification is that it will allow us to focus exclusively on the role of $\nu$ in affecting cross-country differences in output-per-worker. In what follows, we will denote per-worker variables by lowercase letters, for example $y$ and $k$ for output-per-worker and capital-per-worker. Finally we will denote the probability density function of $\nu_{i}$ by $\mu^{\nu}(\cdot)$. 


\section{From Autarky to Free Trade in Intermediate goods}

This section examines how moving from an autarkic equilibrium to a free trade equilibrium in intermediate goods affects the cross-country distribution of output-per-worker. We start by assuming that the world economy is composed of $\mathrm{N}+1$ closed economies that all evolve in autarky and show that in such a case each economy behaves as the standard Solow [1956] growth model with Cobb-Douglas technology and therefore the cross-country distribution of output reflects the shape of the distribution of $\nu$. In particular, we show that if the distribution of $\nu$ - the propensity to favor capital deepening — is uni-modal, then so is the distribution of output-per-worker (and also its logarithm). Furthermore, we show that imperfections in the labor market, as modeled by allowing $x>0$, do not affect the cross-country distribution of income in the autarkic equilibrium. We then open the economies and show how free trade affects the distribution of income. In the absence of imperfections in the labor market, we show why such a model is incapable of explaining the changes in the cross-country distribution of income documented in Section 1.1. In contrast, in the presence of a labor market imperfection which gives rise to a wage premium in the capital intensive firms, we show that the model can explain both an increase in the cross-country dispersion of output-per-worker and potentially cause the emergence of bi-modality.

For each case — the autarkic and free trade equilibrium — we first examine the determination of output taking a country's capital labor ratio as given. Then we study properties of the crosscountry distribution when physical capital is allowed to adjust to its steady state value. As it can be expected, the resulting distributions will be affected by the distribution of $\nu$. We should immediately emphasize that, for clarity of presentation, we are initially assuming that countries differ only in terms of their propensity to accumulate capital $(\nu)$. Obviously, this assumption is at odds with the data since it is well known that differences in $\nu$ can only account for a fraction of the cross-country differences in income levels. For this reason, we will reintroduce differences in labor augmenting knowledge towards the end.

\subsection{Autarky}

In the absence of international trade in intermediate goods, the model we have presented takes a very simple form. In particular, as stated in Proposition 1, aggregate output in the country can be represented by a simple Cobb-Douglas production function. ${ }^{12}$

Proposition 1 In the absence of international trade, the relationship between output-per-worker

\footnotetext{
${ }^{12}$ The interested reader is referred the Appendix for proofs of the Propositions.
} 
and capital-per-worker is given by

$$
y_{i t}=\Upsilon\left(\Gamma_{t}\right) k_{i t}^{\alpha \varphi+\beta(1-\varphi)}
$$

with $\Upsilon\left(\Gamma_{t}\right) \equiv \Theta_{1}^{\varphi} \Theta_{2}^{1-\varphi} \frac{(\alpha \varphi)^{\alpha \varphi}(\beta(1-\varphi))^{\beta(1-\varphi)}}{[\alpha \varphi+\beta(1-\varphi)]^{\alpha \varphi+\beta(1-\varphi)}} \frac{((1-\alpha) \varphi)^{(1-\alpha) \varphi}\left((1-\varphi) \frac{1-\beta}{1+x}\right)^{(1-\beta)(1-\varphi)}}{\left[(1-\alpha) \varphi+(1-\varphi) \frac{1-\beta}{1+x}\right]^{\varphi(1-\alpha)+(1-\varphi)(1-\beta)}} \Gamma_{t}^{1-\alpha \varphi-\beta(1-\varphi)}$

Two aspects are worth emphasizing about Proposition 1. First, to understand this proposition, it is helpful to note that the amount of labor time being allocated by each intermediate good firm between the production of type 1 versus type 2 type intermediate goods does not change as the capital-labor ratio varies. In effect, as the capital-labor ratio increases the production of the type 2 good increases, but because the relative price of $Z_{2}$ in terms of $Z_{1}$ good adjusts, this leaves unchanged the ratio of marginal productivity of labor across the two activities. Therefore, as the capital-labor ratio increases, a country does not become more specialized in the production of the capital intensive good.

A second aspect to note from Proposition 1 is that an increase in $x$ - the labor market distortion - leads to a decrease in $\Upsilon\left(\Gamma_{t}\right)$ and hence a decrease in output-per-worker. This arises due to the fact that the labor market distortion causes a sub-optimal allocation of worker between the two goods $Z_{1}$ and $Z_{2}$. However, it is worth noting that in the autarky equilibrium, the labor market distortion $x$ does not affect the return to capital accumulation and does not cause any discrepancy between the social and private return to capital as stated in the next proposition.

Proposition 2 In the autarky equilibrium, the private and social returns to capital are equalized and are independent of the size the labor market distorsion, $x$.

We now turn to the determination of the cross-country distribution of output-per-worker when we allow capital to adjust to its steady state. Using the capital accumulation equation (7) and the aggregate production function given by Proposition 1, we find that along the steady state growth path $y_{i t}$ satisfies the following relationship:

$$
y_{i}=\Upsilon\left(\Gamma_{t}\right)^{\frac{1}{1-\alpha \varphi-\beta(1-\varphi)}} \nu_{i}^{\frac{\alpha \varphi+\beta(1-\varphi)}{1-\alpha \varphi-\beta(1-\varphi)}}
$$

For ease of comparison with empirical counterparts, it is helpful to consider the determination of the logarithm of $y_{i}$ relative to a reference economy, $y_{0 t}$. To this end, let us define $\widehat{y}_{i}=$ $\log \left(y_{i t}\right)-\log \left(y_{0 t}\right)$ and $\widehat{\nu_{i}} \equiv \log \left(\nu_{i}\right)-\log \left(\nu_{0}\right)$ and note that $\widehat{y}_{i}$ satisfies the follow linear relationship:

$$
\widehat{y}_{i}=\left(\frac{\alpha \varphi+\beta(1-\varphi)}{1-\alpha \varphi-\beta(1-\varphi)}\right) \widehat{\nu}_{i}
$$

It is now straightforward to derive the steady state distribution of $\widehat{y}_{i}$, which is given in the next proposition. 
Proposition 3 In the absence of international trade, the steady state distribution of (log-) outputper-worker relative to a reference economy $\left(y_{0}\right)$, is given by

$$
\mu^{\widehat{Y}}(\widehat{y})=\frac{1-\alpha \varphi-\beta(1-\varphi)}{\alpha \varphi+\beta(1-\varphi)} \mu^{\widehat{\nu}}\left(\frac{1-\alpha \varphi-\beta(1-\varphi)}{\alpha \varphi+\beta(1-\varphi)} \widehat{y}\right)
$$

where $\mu^{\nu}(\cdot)$ denotes the distribution of $\widehat{\nu} \equiv \log (\nu)-\log \left(\nu_{0}\right)$.

As can be seen in Proposition 3, the distribution of relative (log-) output-per-worker consists simply of a re-scaling of the distribution of $\widehat{\nu}$. A direct implication of this linearity in the relationship between $\widehat{\nu_{i}}$ and $\widehat{y}_{i}$ is that if the propensity to favor capital deepening, $\widehat{\nu}$, is distributed according to a uni-modal distribution, then the distribution of output-per-worker inherits this uni-modality under autarky.

\subsection{Free trade and the static determination of output-per-worker}

We now turn to examining the implications of opening up trade in intermediate goods for the equilibrium distribution of output-per-worker. To derive these implications, we need to address two distinct questions. First, we need to determine how a country's aggregate level of output changes when it can trade both type of goods $\left(Z_{2}\right.$ and $\left.Z_{1}\right)$ in the world market at relative price $p$. Second, we need to determine the world relative price $p$. Our approach is to focus on the first question and adopt a convenient approximation for the second. In effect, we will assume that world relative price, $p$, of $Z_{2}$ in terms of $Z_{1}$ under free trade is given by the autarky price of the reference economy. Since in practice, our reference economy is the US economy, this assumption corresponds to assuming that the US economy is not greatly influenced by the possibility of international trade in the sense that US relative prices under autarky would be quite similar to actual US prices. Since the fraction of output directed to international trade in the US over the period examined is rather small, this appears to us as a reasonable benchmark. Nonetheless, in the appendix we show how our results can be extended and our proposition rephrased when this approximation does not hold.

In order to characterize the main properties of the economy, it is helpful to first make explicit the conditions that would lead a country to full specialization in the production of either $Z_{1}$ or $Z_{2}$ under free trade. This is given in Lemma 1.

Lemma 1 For given international prices, there exists two levels of capital-per-worker denoted by $\underline{k}\left(p_{t}, \Gamma_{t}\right)$ and $\bar{k}\left(p_{t}, \Gamma_{t}\right)$, such that endowed with a capital-per-worker below $\underline{k}\left(p_{t}, \Gamma_{t}\right)$, a small open economy specializes in the production of good 1, while it specializes in the production of 
good 2 when its capital-per-worker lies above $\bar{k}\left(p_{t}, \Gamma_{t}\right)$, with

$$
\begin{aligned}
& \underline{k}\left(p_{t}, \Gamma_{t}\right)=\Gamma_{t}\left(p_{t} \frac{\Theta_{2}}{\Theta_{1}}\right)^{\frac{1}{\alpha-\beta}}\left(\frac{\beta}{\alpha}\right)^{\frac{\beta}{\alpha-\beta}}\left(\frac{1-\beta}{(1-\alpha)(1+x)}\right)^{\frac{1-\beta}{\alpha-\beta}} \\
& \bar{k}\left(p_{t}, \Gamma_{t}\right)=\Gamma_{t}\left(p_{t} \frac{\Theta_{2}}{\Theta_{1}}\right)^{\frac{1}{\alpha-\beta}}\left(\frac{\beta}{\alpha}\right)^{\frac{\alpha}{\alpha-\beta}}\left(\frac{1-\beta}{(1-\alpha)(1+x)}\right)^{\frac{1-\alpha}{\alpha-\beta}}
\end{aligned}
$$

The intuition for Lemma 1 is standard from Hecksher-Ohlin international trade theory: economies specialize in the production of the good which is intensive in the factor they have in abundance. Hence, when the economy is either poorly endowed in capital-per-worker due to insufficient capital accumulation $\left(k_{t}<\underline{k}\left(p_{t}, \Gamma_{t}\right)\right)$, it specializes in the production of good 1 . Conversely, if it accumulates a sufficiently high level of capital per worker $\left(k_{t}>\bar{k}\left(p_{t}, \Gamma_{t}\right)\right)$, it specializes in the production of good 2, which is capital intensive. Given this notation, we can now present the relationship between a country's capital labor ratio and its level of production, which we will refer to as the country's effective production function.

Proposition 4 Under free trade a country's level of output-per-worker is given by

$$
y_{i t}= \begin{cases}\Phi p_{t}^{\varphi-1} A_{1 t} k_{i t}^{\alpha} \Gamma_{t}^{1-\alpha} & \text { if } k_{i t} \leqslant \underline{k}\left(p_{t}, \Gamma_{t}\right) \\ A\left(p_{t}\right) k_{i t}+B\left(p_{t}\right) \Gamma_{t} & \text { if } \underline{k}\left(p_{t}, \Gamma_{t}\right) \leqslant k_{i t} \leqslant \bar{k}\left(p_{t}, \Gamma_{t}\right) \\ \Phi p_{t}^{\varphi} A_{2 t} k_{i t}^{\beta} \Gamma_{t}^{1-\beta} & \text { if } k_{i t} \geqslant \bar{k}\left(p_{t}, \Gamma_{t}\right)\end{cases}
$$

where $\Phi=\varphi^{\varphi}(1-\varphi)^{1-\varphi}$ and

$$
\begin{aligned}
& A\left(p_{t}\right)=\Phi p_{t}^{\varphi-1} \frac{\alpha\left(1-\alpha-\frac{1-\beta}{1+x}\right)}{\beta(1-\alpha)-\alpha \frac{1-\beta}{1+x}}\left(p_{t} \Theta_{2}\right)^{\frac{1-\alpha}{\beta-\alpha}} \Theta_{1}^{\frac{1-\beta}{\alpha-\beta}}\left(\frac{\beta}{\alpha}\right)^{\frac{\beta(1-\alpha)}{\beta-\alpha}}\left(\frac{1-\beta}{(1+x)(1-\alpha)}\right)^{\frac{(1-\beta)(1-\alpha)}{\beta-\alpha}} \\
& B\left(p_{t}\right)=\Phi p_{t}^{\varphi-1} \frac{(\beta-\alpha)(1-\alpha)}{\beta(1-\alpha)-\alpha \frac{1-\beta}{1+x}}\left(p_{t} \Theta_{2}\right)^{\frac{\alpha}{\alpha-\beta}} \Theta_{1}^{\frac{\beta}{\beta-\alpha}}\left(\frac{\beta}{\alpha}\right)^{\frac{\alpha \beta}{\alpha-\beta}}\left(\frac{1-\beta}{(1+x)(1-\alpha)}\right)^{\frac{\alpha(1-\beta)}{\alpha-\beta}}
\end{aligned}
$$

This proposition is illustrated in Figure 3, where the production function is depicted under both autarky and free trade. In the autarkic situation, the technology is strictly concave, as implied by Proposition 1. When countries can trade in intermediate goods, the shape of the effective production function depends on whether or not there is a labor market distortion. In particular, in the case where $x=0$, free trade leads to an unambiguous increase in production outcomes as a function $k$. This reflects the standard gains from trade result in international trade theory. In contrast, when $x>0$, Figure 3 indicates that a country's effective production function pivots around the production outcome of the large economy, causing countries with capital-labor ratios lower than that of the large reference economy to potentially loose from international trade. The source of the potential loss comes from the fact that countries with low capital-labor ratios shift their production mix away from the production of $Z_{2}$ towards the production of $Z_{1}$. Since firms producing $Z_{2}$ pay higher wages and thereby have higher labor 
Figure 3: Aggregate Production Function

(a) No wage distortion $(x=0$ or $\theta \rightarrow \infty)$

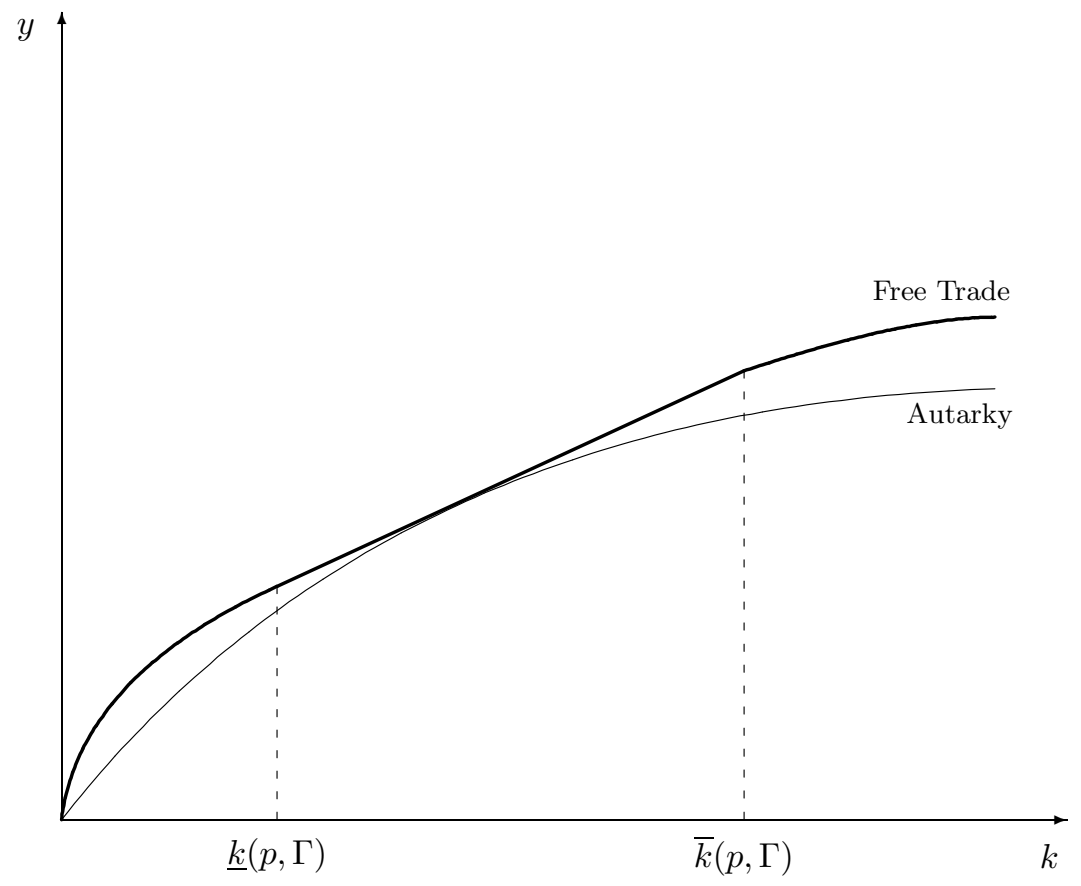

(b) Wage premium associated with $Z_{2}(x>0)$

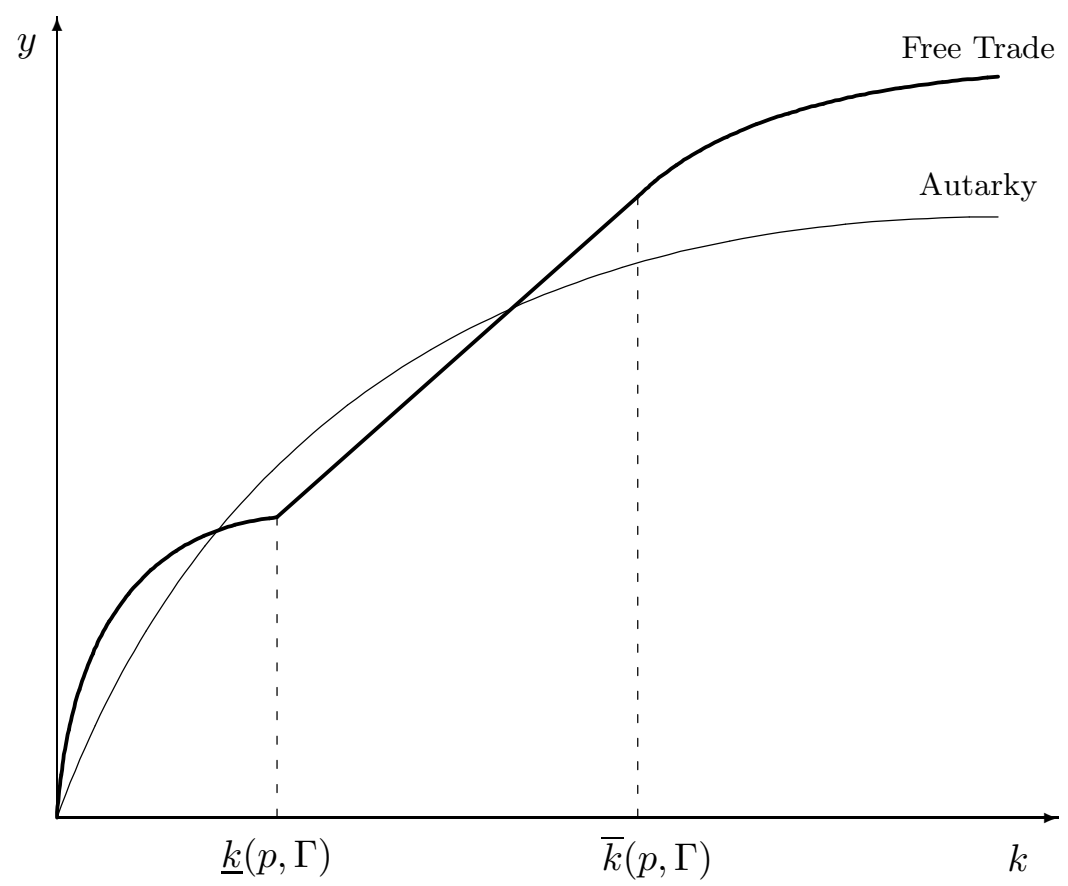


productivity, this shift in production mix can cause a fall in output. On the alternative side, countries with high capital-labor ratio will fare particularly well under free trade since they specialize in the high-wage high-labor productivity jobs. ${ }^{13}$

An important element to acknowledge is that, even when $x>0$, free trade in intermediate goods causes factor price equalization for all non-fully specialized countries. However, this does not imply that the average wage will be equal across such countries. It only implies that the sector specific wages $\left(W_{1}\right.$ and $\left.W_{2}\right)$ and the rental price of capital are equalized. The average wage will vary across country due to the different output compositions. Hence, when $x>0$, an increase in the capital-labor ratio does not only increase output-per-worker but also increases average wages as more workers are paid a wage premium. Since this arises with the return on capital being fixed, it implies that under free trade there emerges an externality associated with capital accumulation that was not present under autarky. This property is stated in the following proposition.

Proposition 5 If $x>0$, then under free trade the social returns to capital are higher than the private return for all countries that do not fully specialize. Moreover, the difference between the private and social return to capital is increasing in $x$.

Having established how free trade affects a country's production possibility set, we now investigate the effect of free trade on the steady state distribution of output-per-worker.

\subsection{Free Trade and the steady state Distribution of Output-per-Worker}

This section shows how the opening of trade in intermediates can lead to an increase in the crosscountry dispersion of output-per-worker and, in addition, potentially causes the emergence of bi-modality. We again consider both the cases where $x>0$ and where $x=0$. Since, as can be seen in Figure 3, the effective production function is not concave when $x>0$, it is first necessary to establish that the steady state level of output-per-worker is uniquely defined.

Proposition 6 Under free trade, regardless of the value of $x$, all economies possess a unique nontrivial steady state.

Proposition 6 indicates that the mapping between $\widehat{\nu}_{i}$ and $\widehat{y}_{i}$ will be well-behaved regardless of the value of $x$. The next lemma presents this mapping.

\footnotetext{
${ }^{13}$ It is worth noting that that countries with very low capital-labor ratios are still likely to gain from free trade even in the presence of a labor market imperfection since such countries produced only small quantities of $Z_{2}$ initially and therefore gain by being able to import $Z_{2}$ at a low price relative to their autarky price.
} 
Lemma 2 The relationship between the steady state level of relative output-per-worker in economy $i, \widehat{y}_{i}$, and the relative propensity for capital deepening, $\widehat{\nu}_{i}$ is given by

$$
\widehat{y}_{i}= \begin{cases}\log \left(\frac{(1-\alpha) \varphi+(1-\varphi) \frac{1-\beta}{1+x}}{1-\alpha}\right)+\frac{\alpha}{1-\alpha}\left(\widehat{\nu}_{i}-\widehat{\widehat{\nu}}\right) & \text { if } \widehat{\nu}_{i}<\underline{\widehat{\nu}} \\ \log (\rho)-\log \left(1-(1-\rho) \exp \left(\widehat{\nu}_{i}\right)\right) & \text { if } \underline{\underline{\nu}} \leqslant \widehat{\nu}_{i} \leqslant \overline{\widehat{\nu}} \\ \log \left(\frac{(1-\alpha) \varphi+(1-\varphi) \frac{1-\beta}{1+x}}{\frac{1-\beta}{1+x}}\right)+\frac{\beta}{1-\beta}\left(\widehat{\nu}_{i}-\overline{\widehat{\nu}}\right) & \text { if } \widehat{\nu}_{i}>\overline{\widehat{\nu}}\end{cases}
$$

where $\underline{\widehat{\nu}}=\log \left(\frac{\alpha}{\alpha \varphi+\beta(1-\varphi)}\right)$ and $\overline{\widehat{\nu}}=\log \left(\frac{\beta}{\alpha \varphi+\beta(1-\varphi)}\right)$ and $\rho=\frac{(\beta-\alpha)\left(\varphi(1-\alpha)+(1-\varphi) \frac{1-\beta}{1+x}\right)}{\beta(1-\alpha)-\alpha \frac{1-\beta}{1+x}}$

Endowed with lemma 2 and a distribution for the propensity to accumulate capital, $\widehat{\nu}$, a direct application of the change in variable formula allows for an analytical derivation of the distribution of output-per-worker across countries, as given below.

Proposition 7 The steady state distribution of output-per-worker, relative to the reference economy, $\widehat{y}$, is given by

$$
\mu^{\widehat{Y}}(\widehat{y})= \begin{cases}\frac{1-\alpha}{\alpha} \mu_{\widehat{\nu}}\left(\frac{1-\alpha}{\alpha}(\widehat{y}-\underline{\widehat{y}})-\log \left(\frac{\alpha}{\alpha \varphi+\beta(1-\varphi)}\right)\right) & \text { if } \widehat{y}<\underline{\widehat{y}} \\ \frac{(1-\rho) \exp (\widehat{y})}{1-(1-\rho) \exp (\widehat{y})} \mu_{\widehat{\nu}}\left(\log \left(\frac{1-(1-\rho) \exp (\widehat{y})}{\rho}\right)\right) & \text { if } \underline{y} \leqslant \widehat{y} \leqslant \overline{\hat{y}} \\ \frac{1-\beta}{\beta} \mu_{\widehat{\nu}}\left(\frac{1-\beta}{\beta}(\widehat{y}-\overline{\widehat{y}})-\log \left(\frac{\beta}{\alpha \varphi+\beta(1-\varphi)}\right)\right) & \text { if } \widehat{y}>\overline{\widehat{y}}\end{cases}
$$

where $\mu_{\widehat{\nu}}(\cdot)$ is the distribution of $\widehat{\nu}=\log (\nu)-\log \left(\nu_{0}\right), \rho \equiv \frac{(\alpha \varphi+\beta(1-\varphi))\left(1-\alpha-\frac{1-\beta}{1+x}\right)}{\beta(1-\alpha)-\alpha \frac{1-\beta}{1+x}}$,

$$
\underline{\hat{y}}=\log \left(\frac{\varphi(1-\alpha)+(1-\varphi) \frac{1-\beta}{1+x}}{1-\alpha}\right) \text { and } \overline{\widehat{y}}=\log \left(\frac{\varphi(1-\alpha)+(1-\varphi) \frac{1-\beta}{1+x}}{\frac{1-\beta}{1+x}}\right)
$$

In principal, we can now compare how the distribution given in the previous proposition differs from the one derived under free trade. However, such a comparison is not transparent. In order to make progress on this issue, it is helpful to begin by focusing the analysis of the mapping between $\widehat{\nu}$ and $\widehat{y}$ and identify the first order effects. To this end, Proposition 8 highlights the first order effects of free trade on the mapping between $\widehat{y}_{i}$ and $\widehat{\nu}_{i}$.

Proposition 8 If $x>0$, the first order effect of free trade is to increase the sensitivity of $\widehat{y}_{i}=$ $\log \left(y_{i}\right)-\log \left(y_{0}\right)$ with respect to $\widehat{\nu}_{i}=\log \left(\nu_{i}\right)-\log \left(\nu_{0}\right)$. However, if $x=0$, free trade has no first order effect on the mapping from $\widehat{\nu_{i}}$ to $\widehat{y_{i}}$.

In order to visualize the content of Proposition 8, Figure 4 illustrates how the mapping between $\widehat{y}_{i}$ and $\widehat{\nu}_{i}$ is affected by the opening of trade under both the scenario where $x=0$ and the case 
where $x>0$. As can be seen from the figure, in the case of autarky, the mapping between $\widehat{y}_{i}$ and $\widehat{\nu}_{i}$ is linear with a positive slope regardless of the value of $x$. In the case where $x>0$, the mapping becomes S-shaped, with a crossing occurring at the point $\widehat{\nu}_{i}=0$. The important aspect to note is that at this point, the sensitivity of $\widehat{y}_{i}$ to $\widehat{\nu}_{i}$ (evaluated at $\widehat{\nu}_{i}=0$ ) is strictly greater under free trade relative to autarky. In effect, under autarky

$$
\left.\frac{\partial \widehat{y}_{i}}{\partial \widehat{\nu}_{i}}\right|_{\widehat{\nu}_{i}=0}=\frac{\partial \widehat{y}}{\partial \widehat{\nu}}=\frac{\alpha \varphi+\beta(1-\varphi)}{1-\alpha \varphi-\beta(1-\varphi)},
$$

while under free trade

$$
\left.\frac{\partial \widehat{y}_{i}}{\partial \widehat{\nu}_{i}}\right|_{\widehat{\nu}_{i}=0}=\left(\frac{\alpha \varphi+\beta(1-\varphi)}{1-\alpha \varphi-\beta(1-\varphi)}\right) \frac{(1-\alpha \varphi-\beta(1-\varphi))\left(1-\alpha-\frac{1-\beta}{1+x}\right)}{(\beta-\alpha)\left((1-\alpha) \varphi+(1-\varphi) \frac{1-\beta}{1+x}\right)}>\frac{\alpha \varphi+\beta(1-\varphi)}{1-\alpha \varphi-\beta(1-\varphi)} .
$$

Hence, for countries that do not differ much in terms of $\nu$ relative to the reference economy, there is an unambiguous first order effect due to the opening up of trade since it increases the sensitivity of $\widehat{y}_{i}$ to $\widehat{\nu}_{i}$. In contrast, for the case where $x=0$, we can see that the passage to free trade causes the mapping between $\widehat{y}_{i}$ and $\widehat{\nu}_{i}$ to become convex with a tangency arising at the point where $\widehat{\nu}=0$. Hence, in this case, the passage to free trade has no first order effect on the determination of $\widehat{y}_{i}$ since the sensitivity of $\widehat{y}_{i}$ to $\widehat{\nu}_{i}$ is unchanged in the neighborhood of $\widehat{\nu}_{i}=0$. In order to get a sense of the extent to which the opening up of trade can increase the sensitive of $\widehat{y}_{i}$ to $\widehat{\nu}_{i}$, which is given by the term $\frac{(1-\alpha \varphi-\beta(1-\varphi))\left(1-\alpha-\frac{1-\beta}{1+x}\right)}{(\beta-\alpha)\left((1-\alpha) \varphi+(1-\varphi) \frac{1-\beta}{1+x}\right)}$, it is of interest to give illustrative values to the parameters for $\alpha, \beta, \varphi$ and $x$. For example, in the case where ${ }^{14} \alpha=0.31, \beta=0.4$, $\varphi=0.75$ and $x=0.16$, the effect of opening up trade in intermediate goods would be to double the sensitivity of $\widehat{y}_{i}$ to $\widehat{\nu}_{i}$. Hence, we see that the theoretical effect implied by Proposition 8 can easily be of a sizable magnitude. ${ }^{15}$

The economic mechanism which gives rise to these first order effects should now be quite clear. In particular, under free trade, a country with a high value of $\widehat{\nu}$ (i.e. a high propensity for capital deepening) will gain relative to a country with a low value of $\widehat{\nu}$ since the former will shift its production mix towards the rent-paying high-labor-productivity activity $Z_{2}$, while the latter shifts in the opposite direction and reduces its employment in the rent-paying high-laborproductivity activity. In contrast, in the case where $x=0$, there is no preferable direction in terms of production mix and therefore countries with positive versus negative values of $\widehat{\nu}$ will have to a first approximation similar outcomes. The one last element worth noting from Figure

\footnotetext{
${ }^{14}$ These parameters are chosen so that the share of capital in a closed economy is $1 / 3$.

${ }^{15}$ The model also has implications for how the opening up of trade affects the distribution of measured total factor productivity across countries. In particular, it is easy to show that if $x>0$, then the opening up of trade will increase the dispersion of measured total factor productivity across countries due to the fact that the share of revenue received by capital does not capture the full impact of capital on output, and correspondingly the non-appropriated impact of capital on output is reflected in measured TFP. In contrast, if $x=0$, , then the opening up of trade should have no impact on measured TFP since the market return to capital appropriately captures the marginal productivity of capital.
} 
Figure 4: Output-per-worker versus $\nu$

(a) $x=0$

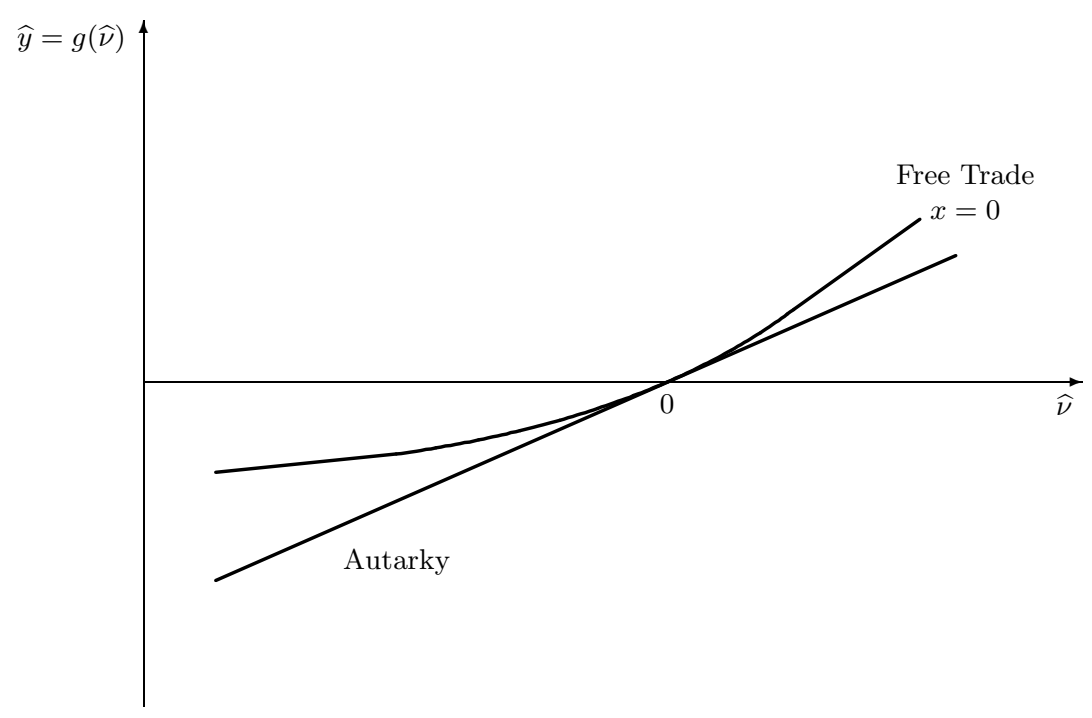

(b) $x>0$

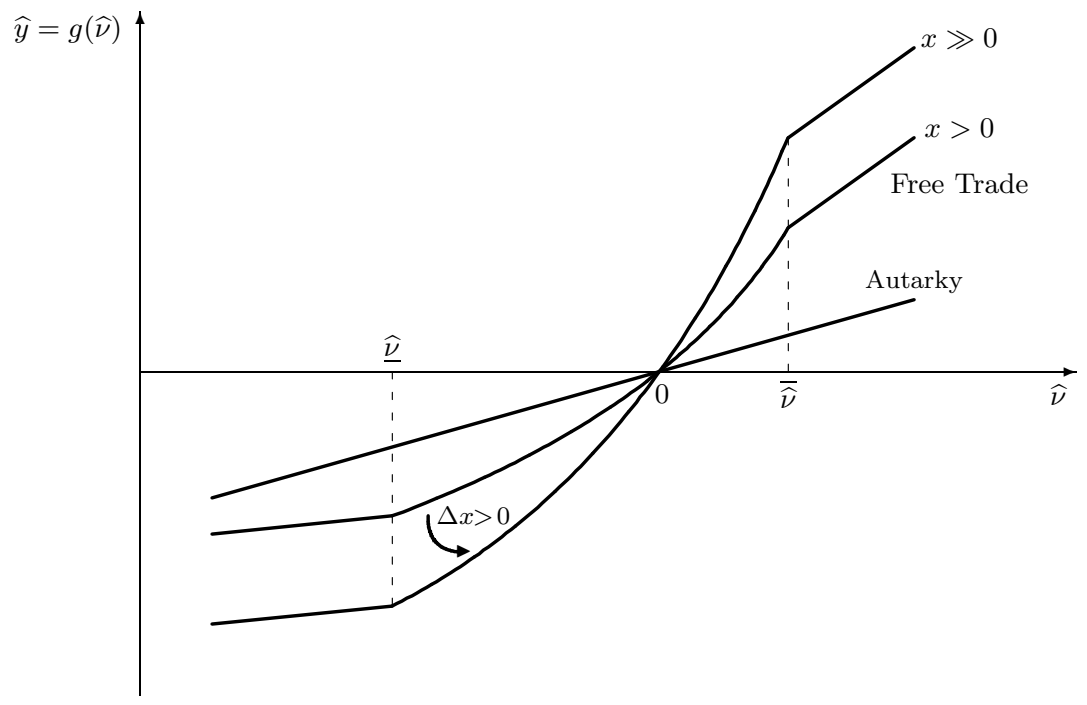


4 is that under free trade the slope of the relationship between $\widehat{y}_{i}$ and $\widehat{\nu}_{i}$ near $\widehat{\nu}_{i}=0$ is strictly increasing in $x$.

Given the content of Proposition 8, it is straightforward to state the first order effects of free trade on the distribution of $\widehat{y}$, as given in following corollary.

Corollary 1 If the distribution of $\widehat{\nu}_{i}$ is concentrated around zero, then the first order effect of free trade on the cross-country distribution of output-per-worker is nil when $x=0$. In contrast, it leads to an increase in dispersion when $x>0$.

The above corollary makes explicit the sense in which the process of globalization may offer an explanation to the observed widening of the cross-country distribution of output-per-worker. However, in addition to potentially explaining an increase in dispersion, it is of interest to examine whether the current model could also explain a change in shape of the distribution in the form of the emergence of bi-modality. The following Lemma is useful for exploring this possibility.

Lemma 3 Consider two observable random variables, $Y_{1}$ and $Y_{2}$, which are both transformations of a random variables $Z$, where the first transformation is linear and results in the variable $Y_{1}=\alpha Z, \alpha>0$, while the second transformation is non-linear (continuous and differentiable) and results in the variable $Y_{2}=g(Z), g^{\prime}(\cdot)>0$. If the distribution of $Y_{1}$ is uni-modal, then a necessary condition for the distribution of $Y_{2}$ to be bi-modal is that $g(\cdot)$ not be a convex function.

This lemma can be directly used to evaluate whether free trade can cause the emergence of bi-modality once one recognizes that the variable $Y_{1}$ can be interpreted as $\widehat{y}_{i}$ under autarky, while $Y_{2}$ can be interpreted as $\widehat{y}_{i}$ under free trade. Hence, the necessary condition for free trade to cause the emergence of bi-modality is that the mapping between $\widehat{y}_{i}$ and $\widehat{\nu}_{i}$ be nonconvex. As we have seen in Figure 4, if $x=0$, the mapping between $\widehat{y}_{i}$ and $\widehat{\nu}_{i}$ is convex and therefore the opening up of trade cannot explain the emergence of a bi-modal distribution. In contrast, if $x>0$, then the mapping between $\widehat{y}_{i}$ and $\widehat{\nu}_{i}$ is not convex (it is S-shaped), and hence the opening up of trade under the presence of labor market imperfections could cause the emergence of bi-modality. This observation is summarized in the following proposition.

Proposition 9 If $x=0$, the opening of trade in intermediate goods cannot generate the emergence of a bi-modality in the cross-country distribution of output-per-worker. Conversely, if $x>0$ then the opening up of trade may cause the distribution to exhibit bi-modality.

Once again, we see that in the absence of labor market imperfections, the process of globalization as modeled in this paper does not offer a very likely candidate for the observed changes in 
the world distribution of output-per-worker, as long as the attention is focused on the $g_{t}(\cdot)$ channel described in Section 1.2. However, once a labor market distortion in the form of a wage premium in high-capital intensive jobs is introduced, then the model offers a potential explanation. Obviously, Proposition 9 only indicates that the opening up of trade may cause the emergence of bi-modality if $x>0$; it does not say that it will cause bi-modality. Nonetheless, we are content with this possibility results ${ }^{16}$ since (i) we know through simple examples that the model can give rise to the emergence of bi-modality, (ii) we will explore empirically whether the mechanism emphasized by this model could explain the observed change in shape of the cross-country distribution of output-per-worker.

At this point it seems appropriate to contrast the above results to those that would be obtained in a model with optimizing consumers as in Ventura [1997]. In Ventura [1997], each country is characterized by infinity-lived optimizing consumer with a country specific discount factor. In this case, the first order effect of opening up of trade is to eliminate decreasing returns to capital but not to increase its average return. Therefore, countries with low discount factors increase there savings rate and thereby experience high investment rates without encountering diminishing returns, while countries with high discount factors reduce investment rates. The resulting differences in investment rates lead to continually expanding differences in capital output ratios across countries. This leads to greater dispersion in the capital-output ratios and therefore to greater dispersion in output-per-worker. In other words, in Ventura's model, globalization affects the cross-country distribution of output-per-worker mainly by changing the distribution of $\nu$. In the framework of section 1.2, this would amount to a change in the $h_{t}(\cdot)$ function.

In contrast, in our model with $x>0$ - even if the distribution of capital-output ratios is held fixed - globalization increases the social returns to being capital abundant, thereby causing increased dispersion even in the absence of any change in capital-output ratios across counties. Hence, globalization affects the distribution of output-per-worker through a change in the sensitivity of output-per-worker with respect to $\nu{ }^{17}$

The differences between these mechanisms can be summarized by an increase in the dispersion of $\nu$ (a change in $h_{t}(\cdot)$ in Ventura's model) as opposed to an increase in the returns to $\nu$ (a change in $g_{t}(\cdot)$ in our model). Each of this mechanism can be evaluated empirically, that is, the quantitative relevance of these different mechanisms can be potentially evaluated by examining

\footnotetext{
${ }^{16}$ It is possible to derive the stronger result that free trade will cause the emergence of more that one mode in the distribution of $\widehat{y}$ if one assumes that the distribution of $\widehat{\nu}$ is continuous and that its support encompasses $\overline{\widehat{\nu}}$.

${ }^{17}$ In terms of the notation presented in Section 2.1, the central change in our model corresponds to a change in the slope of the function $g_{t}(\cdot)$, while in Ventura the effect on $g_{t}(\cdot)$ is only of second order while the change on $h_{t}(\cdot)$ is of first order.
} 
whether much of the change in the cross-country distribution of income can be accounted for by changes in the distribution of $\nu$ versus changes in the returns to $\nu$. Accordingly, such an exercise will be conducted in Section 4 .

\subsection{Reintroducing Differences in Labor Augmenting Knowledge}

Up to now, we have been abstracting from country specific differences in productivity. In this section we want to briefly discuss how our main results extend if we allow for country specific differences in labor augmenting knowledge -as captured in our notation by $\Omega_{i}$. Recall that in the absence of trade in intermediates, the mapping from $\widehat{\nu_{i}}$ to $\widehat{y}_{i}$ is given by the following:

$$
\widehat{y}_{i}=\left(\frac{\alpha \varphi+\beta(1-\varphi)}{1-\alpha \varphi-\beta(1-\varphi)}\right) \widehat{\nu_{i}}
$$

In the case of free trade, the linear approximation for the mapping between $\widehat{y}_{i}$ and $\widehat{\nu_{i}}$ is given by the following relationship, where it is immediate to see that the sensitivity of $\widehat{y}_{i}$ to $\widehat{\nu_{i}}$ is greater under free trade if $x>0$.

$$
\widehat{y}_{i} \approx\left(\frac{\alpha \varphi+\beta(1-\varphi)}{1-\alpha \varphi-\beta(1-\varphi)}\right) \frac{(1-\alpha \varphi-\beta(1-\varphi))\left(1-\alpha-\frac{1-\beta}{1+x}\right)}{(\beta-\alpha)\left((1-\alpha) \varphi+(1-\varphi) \frac{1-\beta}{1+x}\right)} \widehat{\nu}_{i}
$$

If we now introduce differences across countries in labor augmenting knowledge, then these two mappings need to be modified to reflect the differential productivity term $\widehat{\omega}_{i}=\log \Omega_{i}-\log \Omega_{0}$. In the case of autarky, this implies that the determination of $\widehat{y}_{i}$ is given by:

$$
\widehat{y}_{i}=\left(\frac{\alpha \varphi+\beta(1-\varphi)}{1-\alpha \varphi-\beta(1-\varphi)}\right) \widehat{\nu}_{i}+\widehat{\omega}_{i}
$$

while in the case of free trade, the determination of $\widehat{y}_{i}$ is approximately given by:

$$
\widehat{y}_{i} \approx\left(\frac{\alpha \varphi+\beta(1-\varphi)}{1-\alpha \varphi-\beta(1-\varphi)}\right) \frac{(1-\alpha \varphi-\beta(1-\varphi))\left(1-\alpha-\frac{1-\beta}{1+x}\right)}{(\beta-\alpha)\left((1-\alpha) \varphi+(1-\varphi) \frac{1-\beta}{1+x}\right)} \widehat{\nu}_{i}+\widehat{\omega}_{i}
$$

Therefore, in the absence of any change in the distribution of $\widehat{\nu_{i}}$ or $\widehat{\omega}_{i}$, the first order effect of opening up of trade in intermediates will be to increase the dispersion of $\widehat{y}_{i}$ if $\widehat{\nu}_{i}$ and $\widehat{\omega}_{i}$ are uncorrelated or positively correlated. Hence, if we allow for cross-country differences in $\Omega_{i}$, our main results continue to hold quite generally. It is only in the case of a sufficiently strong negative correlation between $\widehat{\nu}_{i}$ and $\widehat{\omega}_{i}$ — which a priori seems rather unlikely — where the introduction of differences in $\Omega_{i}$ limits the scope of application of our main results.

\section{Empirical Evaluation}

The model of the previous section illustrated how the opening up of trade can affect the crosscountry distribution of income when workers receive rents in capital intensive jobs. In this 
section we explore the empirical relevance of the model by examining four key implications. First, the model implies that during a period of globalization we should observe an increase in the social returns to capital accumulation, that is, $\frac{\partial \widehat{y}_{i}}{\partial \widehat{\nu}_{i}}$ should increase over a period of globalization. To explore this prediction, we will estimate and compare the mapping between $\widehat{\nu}_{i}$ and $\widehat{y}_{i}$ in the period 1978-98 versus the period 1960-78 using different instrumental variable approaches. Second, if the model is right, then it is among countries most likely affected by trade where we should observe an increase in the returns to capital accumulation. We will explore this implication by comparing the behavior of the sub-sample of more open countries relative to the less open countries. Third, the model implies that the increase returns to capital should account for much of the observed change in the cross-country distribution of income. This implication will be examined by building a counter-factual distribution of output-per-worker in 1998 where we undo the effects of any change in $\frac{\partial \widehat{y}_{i}}{\partial \widehat{\nu}_{i}}$ observed over the period 1978-98 versus 1960-78. If changes in $\frac{\partial \widehat{y}_{i}}{\partial \widehat{\nu}_{i}}$ are central for understanding the observed change in cross-country distribution of output-per-worker, then such a counterfactual should replicate rather well the observed 1978 distribution. Fourth, the model predicts that during the period of globalization, countries which favor capital accumulation ( i.e. countries with high values of $\nu_{i}$ ) should experience stronger employment growth in high capital intensive sectors. We examine this implication by comparing the growth in manufacturing employment between between high and low $\nu_{i}$ countries.

\subsection{Has There Been an Increase in the Social Return to Capital Accumula- tion?}

As highlighted by Proposition 8 and Equations (11) and (12), a central implication of our model is that the first order effect of opening up trade is to increase the effect of $\widehat{\nu}_{i}$ on the steady state value of $\widehat{y}_{i}$. To examine the empirical relevance of this idea, it is appropriate to consider a partial adjustment model for $\widehat{y}_{i}$, where $\widehat{y}_{i}$ converges at rate $\lambda_{t}$ towards its steady state value. In this case, the relationship between the growth in $\widehat{y}_{i}$ and $\widehat{\nu}_{i}$ is approximately given by

$$
\widehat{y}_{i, t+1}-\widehat{y}_{i, t}=\left(\lambda_{t}-1\right) \widehat{y}_{i t}+\left(1-\lambda_{t}\right) \eta_{t} \widehat{\nu}_{i, t}
$$

where the model predicts that $\beta_{t}$ should be greater in a more globalized environment than under a situation closer to autarky. ${ }^{18}$ In order to evaluate this prediction, we estimate Equation (15) over separately over the period 1960-78 and the period 1978-98, and we examine whether the results give support to the idea of an increase in the sensitivity of $\widehat{y}_{i}$ to $\widehat{\nu}_{i}$. Table 3 and 4 report results based on estimating Equation (15) by OLS and instrumental variables. The data used in the regression are taken from the World Penn Tables 6.0, and the set of countries used in estimation are the same as that underlying Figure 1. The value of $\widehat{\nu}_{i t}$ for each country is

\footnotetext{
${ }^{18}$ Note that Equation (15) can be seen as simply a case of a growth regression with time-varying coefficients.
} 
constructed as in Mankiw et al. [1992] by setting $\gamma+\delta$ at $5 \%$ and using the average value of investment-output ratio over the relevant sub-period for $s_{i}$, and using the average growth of the adult population over the relevant sub-period for $n_{i}$. This variable, and all other variables, are then expressed as deviation from the US. In columns (1) and (2) of Table 3, we report the OLS

Table 3: Benchmark Regression

\begin{tabular}{lcccccccc}
\hline \hline & \multicolumn{2}{c}{ OLS } & \multicolumn{2}{c}{ IV1 } & \multicolumn{3}{c}{ IV1 } & \multicolumn{3}{c}{ IV2 } \\
& $60-78$ & $78-98$ & $60-78$ & $78-98$ & $60-78$ & $78-98$ & $60-78$ & $78-98$ \\
& $(1)$ & $(2)$ & $(3)$ & $(4)$ & $(5)$ & $(6)$ & $(7)$ & $(8)$ \\
\hline Cst. & -0.004 & -0.013 & -0.002 & -0.013 & -0.001 & -0.013 & 0.000 & -0.012 \\
& $(0.003)$ & $(0.003)$ & $(0.004)$ & $(0.003)$ & $(0.005)$ & $(0.003)$ & $(0.004)$ & $(0.003)$ \\
$\widehat{y}_{0}$ & -0.013 & -0.014 & -0.011 & -0.014 & -0.015 & -0.016 & -0.014 & -0.016 \\
& $(0.002)$ & $(0.002)$ & $(0.003)$ & $(0.003)$ & $(0.003)$ & $(0.003)$ & $(0.003)$ & $0.003)$ \\
$\widehat{\nu}$ & 0.016 & 0.029 & 0.011 & 0.030 & 0.010 & 0.031 & 0.008 & 0.030 \\
& $(0.003)$ & $(0.003)$ & $(0.004)$ & $(0.005)$ & $(0.005)$ & $(0.005)$ & $(0.004)$ & $(0.005)$ \\
$\widehat{H}$ & - & - & - & - & 0.005 & 0.003 & 0.006 & 0.003 \\
& & & & & $0.003)$ & $(0.004)$ & $(0.003)$ & $(0.004)$ \\
$R^{2}$ & 0.36 & 0.51 & - & - & - & - & - & - \\
$R_{\mathrm{FS}}^{2}$ & - & - & 0.58 & 0.62 & 0.62 & 0.65 & 0.82 & 0.75 \\
\hline $\mathcal{Q}($ Total $)$ & 12.859 & {$[0.002]$} & 9.665 & {$[0.008]$} & 13.467 & {$[0.004]$} & 16.768 & {$[0.001]$} \\
$\mathcal{Q}\left(y_{0}\right)$ & 0.017 & {$[0.898]$} & 0.501 & {$[0.479]$} & 0.100 & {$[0.752]$} & 0.175 & {$[0.676]$} \\
$\mathcal{Q}(\nu)$ & 9.999 & {$[0.002]$} & 7.371 & {$[0.007]$} & 7.019 & {$[0.008]$} & 11.871 & {$[0.001]$} \\
$\mathcal{Q}(H)$ & - & - & - & - & 0.167 & {$[0.683]$} & 0.218 & {$[0.640]$} \\
\hline \hline
\end{tabular}

Note: Standard errors in parenthesis, $\mathrm{p}$-values in brackets. IV1: the variables used to instrument $\widehat{\nu}$ the average (c/y) over the sub-sample, and the average growth rate of population over the 15 first periods of the sub-sample. IV2: The intrument is the beginning of period investment-tooutput ratio. 75 observations. 68 observations when education is included. $R_{\mathrm{FS}}^{2}$ denotes the $R^{2}$ of the first stage regression.

estimate of Equation (15) along with stability tests for the coefficients. As can be seen, the OLS estimates suggest that the relationship between the growth of $\widehat{y}_{i}$ and $\widehat{\nu}_{i}$ changed between the two periods, while the dependence of growth on initial conditions is found to be stable. In effect, the stability test, $\mathcal{Q}(\widehat{\nu})$ indicates that the coefficient on $\widehat{\nu}$ changes significantly when comparing the period $1960-78$ versus $1978-98(\mathrm{p}-\text { value }=0.002)^{19}$, while $\mathcal{Q}\left(\widehat{y}_{0}\right)$ indicates that stability of the coefficient on $\widehat{y}_{0}(\mathrm{p}$-value $=0.898) .{ }^{20}$ The coefficient on $\widehat{\nu}$ is estimated to be 0.016 over the period 1960-78, while its estimate almost doubles to the value of 0.029 for the period 1978-98.

Since $\widehat{\nu}_{i, t}$ may be correlated with other factors affecting the growth in output-per-worker, in the last columns of Table 3 and in Table 4, we report a set of instrumental variable estimates of Equation (15). In particular, in column (3) and (4) (IV1) of Table 3, we instrument $\widehat{\nu}_{i, t}$ with

\footnotetext{
${ }^{19}$ The test of the overall stability of all three coefficients $(\mathcal{Q}$ (Total)) is also clearly rejected.

${ }^{20}$ Note that all our stability tests are performed allowing for residuals to be correlated within countries over the two samples and for the variances to differ.
} 
two instruments: ${ }^{21}$ the country's average ratio of personal consumption to GDP, which captures the behavior of private savings, and the rate of growth of adult population over the first fifteen years of each sub-sample. ${ }^{22}$ These two instruments are in the spirit of the model since it treats savings behavior and population growth as exogenous to the growth process. As can be seen, the estimates obtained using this set of instruments results in a slightly reduced coefficient on $\widehat{\nu}_{i}$ in the first period, but do not change the coefficient in the second period, leaving unchanged the observation of a substantial and significant increase in the sensitivity of $\widehat{y}_{i}$ to $\widehat{\nu}_{i}$ between the two periods. In columns (5) and (6) of the table, in addition to instrumenting $\widehat{\nu}_{i}$ as in the previous experiment, we include as an additional regressor the average number of years of education in the country. ${ }^{23}$ The inclusion of this measure of human capital does not affect the observed pattern regarding the coefficient on $\widehat{\nu}{ }^{24}$ In Columns (7) and (8) of the Table, we instrument $\widehat{\nu}$ using the investment-output ratio in the initial year of each sample period. For example, for the period 1960-78 we use the investment-output ratio in 1960 to instrument $\widehat{\nu}_{i}$. This instrument has the advantage relative to the average savings rate that it is pre-determined relative to each period under study. We also include the average years of education in the regression to control for differences across countries in labor augmenting knowledge. Once again we find a very significant increase in the sensitivity of $\widehat{y}$ to $\widehat{\nu}$ between the period 1960-78 versus 1978-98. ${ }^{25}$

The instruments used in Table 3 can be criticized on the ground that they are unlikely to be truly exogenous and this lack of exogeneity may explain the results. For example, it may be that savings behavior has become more correlated with growth outcomes, and it may be that investment has become better at predicting growth outcome; in which case the instrumental variable estimates would be biased in favor of observing increased sensitivity of $\widehat{y}$ to $\widehat{\nu}$. In order to address such criticism, in Table 4 we instrument $\widehat{\nu}$ using demographic variation across countries. In Columns (1) and (2) of the table we use the rate of growth of adult population over the first fifteen years of each sub-sample to instrument $\widehat{\nu}$. Since an adult is defined as someone over 15 years of age, this variable is likely exogenous with respect to growth developments over the period considered. The most remarkable aspect from this instrumental variable strategy is the similarity in the resulting estimates with respect to those presented in Table 3 . Once

\footnotetext{
${ }^{21}$ The instruments are again expressed as deviations from the US value.

${ }^{22}$ The reason we take the rate of adult population growth over the first fifteen years as an instrument as opposed to taking the rate over the entire sub-period is aimed at correcting for the potential endogeneity of fertility within each window, as it takes at least 15 years for fertility to affect the labor force. However, it is worth noting that our results are almost identical regardless of which if these two measures of adult population growth we use.

${ }^{23}$ This variable is taken from Barro and Lee [1993].

${ }^{24}$ We have also verified the robustness of this pattern with respect to several other measures of human capital.

${ }^{25}$ According to the theory, the regression coefficients in the first period (1960-78) can be used to infer capital shares (as is done in Mankiw, Romer and Weil (1992)). For example, from the IV regressions in the before last column, the implied capital shares is .36 , which is quite reasonable. Note however that we cannot we cannot directly infer capital shares from the regression coefficients in the second period.
} 
Table 4: Robustness to Demographic Instruments

\begin{tabular}{lcccc}
\hline \hline & \multicolumn{2}{c}{ IV3 } & \multicolumn{2}{c}{ IV4 } \\
& $60-78$ & $78-98$ & $60-78$ & $78-98$ \\
& $(1)$ & $(2)$ & $(3)$ & $(4)$ \\
\hline Cst. & -0.000 & -0.019 & 0.001 & -0.014 \\
& $(0.008)$ & $(0.006)$ & $(0.007)$ & $(0.005)$ \\
$\widehat{y}_{0}$ & -0.015 & -0.019 & -0.014 & -0.017 \\
& $(0.004)$ & $(0.004)$ & $(0.004)$ & $(0.004)$ \\
$\widehat{\nu}$ & 0.009 & 0.050 & 0.007 & 0.036 \\
& $(0.010)$ & $(0.014)$ & $(0.009)$ & $(0.012)$ \\
$\widehat{H}$ & 0.005 & -0.004 & 0.006 & 0.001 \\
& $(0.004)$ & $(0.007)$ & $(0.004)$ & $(0.006)$ \\
$R_{\mathrm{FS}}^{2}$ & 0.48 & 0.43 & 0.50 & 0.43 \\
$\mathcal{Q}($ Total $)$ & 12.954 & {$[0.005]$} & 9.315 & {$[0.025]$} \\
$\mathcal{Q}\left(y_{0}\right)$ & 0.695 & {$[0.405]$} & 0.379 & {$[0.538]$} \\
$\mathcal{Q}(\nu)$ & 6.595 & {$[0.010]$} & 4.239 & {$[0.040]$} \\
$\mathcal{Q}(H)$ & 1.755 & {$[0.185]$} & 0.514 & {$[0.473]$} \\
\hline \hline
\end{tabular}

Note: Standard errors in parenthesis, $\mathrm{p}$-values in brackets. IV3: the variable used to instrument $\widehat{\nu}$ is the average growth rate of population over the 15 first periods of the sub-sample. IV4: The instrument is the average annual rate of growth of total population over the period 19601998. 68 observations. $R_{\mathrm{FS}}^{2}$ denotes the $R^{2}$ of the first stage regression. 
again we find that the coefficient on $\widehat{\nu}_{i}$ increases significantly, with the dependence of initial condition remaining very stable; thereby supporting the idea of a substantial increase in the sensitivity of $\widehat{y}_{i}$ to $\widehat{\nu}_{i}$ as implied by the model. One potential criticism of using the rate of growth of adult population over the first fifteen years each sub-sample as an instrument is that it may be contaminated by measurement error since the regressant is constructed using the adult population data. To examine relevance of this issue, in Columns (3) and (4) of Table 4, we instrument $\widehat{\nu}_{i, t}$ using a period invariant demographic instrument which is the average growth of the entire population over the entire period 1960-1998. In other words, we are now using the same demographic instrument to instrument $\widehat{\nu}_{i}$ over the two periods. Hence, unless measurement errors are increasing substantially over time, which seems unlikely, this instrument should be immune to the criticism that measurement error would bias in favor of finding an increase in the sensitivity of $\widehat{y}_{i}$ to $\widehat{\nu}_{i}$. In this case, the coefficient on $\widehat{\nu}_{i}$ goes from 0.007 to 0.036 and this increase is again found statistically significant, as the p-value associated to the test for stability of the coefficient on $\widehat{\nu}$ is 0.040 . Let us note that all the different instruments we used have quite substantial predictive power $\widehat{\nu}$, with $R^{2}$ of the first stage regression ranging mainly between 0.4 and 0.8 , as reported in the line $R_{\mathrm{FS}}^{2}$ of Tables 3 and 4 .

The point we believe that one should take away from these different instrumental variable strategies is that the endogeneity of $\widehat{\nu}$ appears to be quite minor, especially over the second period. Therefore the observed increase in the sensitivity of $\widehat{y}_{i}$ to $\widehat{\nu}_{i}$ may reasonably be interpreted as capturing a change in the causal relationship between $\widehat{\nu}_{i}$ to $\widehat{y}_{i}$. The reason we believe that this is the most plausible interpretation is that we have not found an endogeneity story which can simultaneously explain why all these different instrument variables strategies come up with approximately the same coefficients.

Another potential criticism with the above estimates is that they have been derived without controlling for many other factors that may affect growth. In order to examine this, we have reestimated the above relationships allowing for country fixed effects. These results are presented in Appendix D. ${ }^{26}$ Allowing for country fixed effects is an easy way of verifying the robustness of the above results with respect to omitted (time-invariant) country specific factors such as scale, institutions or location. As is well known in the literature, when we allow for fixed effects, the estimated speed of convergence is increased substantial, and this is precisely what we find. More importantly for the focus of this paper, when we allow for fixed effects, we again find a statistically significant and substantial rise in the sensitivity of $\widehat{y}_{i}$ to $\widehat{\nu}_{i}$, and this rise corresponds again to an approximate doubling of the coefficient.

We have also extensively examined adding additional time-varying regressors to our estimations

\footnotetext{
${ }^{26}$ When we allow for fixed effects, the instrumental variable strategy exploited under IV4 can not be implemented because there is no time variation in the instrument.
} 
of Equation (15). For example, we have verified the robustness of our results against including different measures of human capital, fertility rates, the relative price of equipment goods and non-linear terms in initial conditions. In all of these cases, we constantly found that the coefficient on $\widehat{\nu}$ increased significantly between the two periods and by an order of magnitude similar to that reported here, suggesting that our results are not driven by the omission of widely recognized co-variates of growth .

Even if the results in Table 3 and 4 are all very similar, thereby providing considerable support for the idea that the social returns to capital accumulation increased from the 1960-78 period to the 1978-98 period as implied by the model, it is natural to ask whether these results could be driven by a few outlier countries. To examine this issue, we looked at whether the results were sensitive to varying the sample by excluding subgroups of countries. Without reporting the results here, we examined the robustness of our findings to the following change of sample (i) excluding the Asia tigers from the sample (i.e., Singapore, Taiwan, Hong-Kong and Korea), (ii) excluding the countries of South America, (iii) introducing the countries of sub-Saharan Africa, and (iv) focusing exclusively on the sample of the 18 richest OECD countries. In all these cases, we found the same pattern whereby there is an approximate doubling or tripling of the effect of $\widehat{\nu}$ on $\widehat{y}$ in the later period relative to the early period.

Since Equation (15) is linear in $\widehat{\nu}_{i}$, the above results only provide evidence in favor of the model's first order implication regarding the change in mapping between $\widehat{\nu}_{i}$ and $\widehat{y}_{i}$. However, as can be seen in Figure 4, the model implies more than just a change in slope, it actually implies that the process of globalization should change the mapping between $\widehat{\nu}_{i}$ and $\widehat{y}_{i}$ from a linear mapping to an S-shaped mapping. To explore this possibility, consider the following non-linear generalization of Equation (15):

$$
\widehat{y}_{i, t+1}-\widehat{y}_{i, t}=\left(\lambda_{t}-1\right) \widehat{y}_{i t}+\left(1-\lambda_{t}\right) g_{t}\left(\widehat{\nu}_{i, t}\right)
$$

In order to examine the shape of the the relationship between $\widehat{\nu}_{i}$ and $\widehat{y}_{i}$, i.e. $g_{t}\left(\widehat{\nu}_{i, t}\right)$, in Figure 5 , we plot a non-parametric estimation of the function $g_{t}(\cdot)$ for each subperiod. ${ }^{27}$ The important aspect to note in Figure 5 is that the estimated change in the $g(\cdot)$ function corresponds remarkably closely to that implied by the model as highlighted in Figure 4 when $x>0$. In particular, the estimate of $g(\cdot)$ for 1978-98 indicates a strong increase in slope around the point where $\widehat{\nu}_{i}=0$, while there no such increase in the tails. Recall from Lemma 3 and Proposition 9 that it is precisely a change of shape of this sort which is necessary for the emergence of bi-modality. In comparison, for the 1960-78 period, the relationship between $\widehat{\nu}_{i}$ and $\widehat{y}_{i}$ appears almost perfectly linear. In this sense, Figure 5 provides considerable additional support for the

\footnotetext{
${ }^{27}$ In Figure 5, we are careful to plot the function $g_{t}(\cdot)$ only over the range where there is positive mass in the distribution of $\widehat{\nu}_{i}$. For example, about $50 \%$ of the mass of $\widehat{\nu}_{i}$ lies between -0.5 and 0.3 and the rest of the mass is in the tails.
} 
Figure 5: Non-Parametric Estimate of $g_{t}\left(\widehat{\nu}_{i, t}\right)$

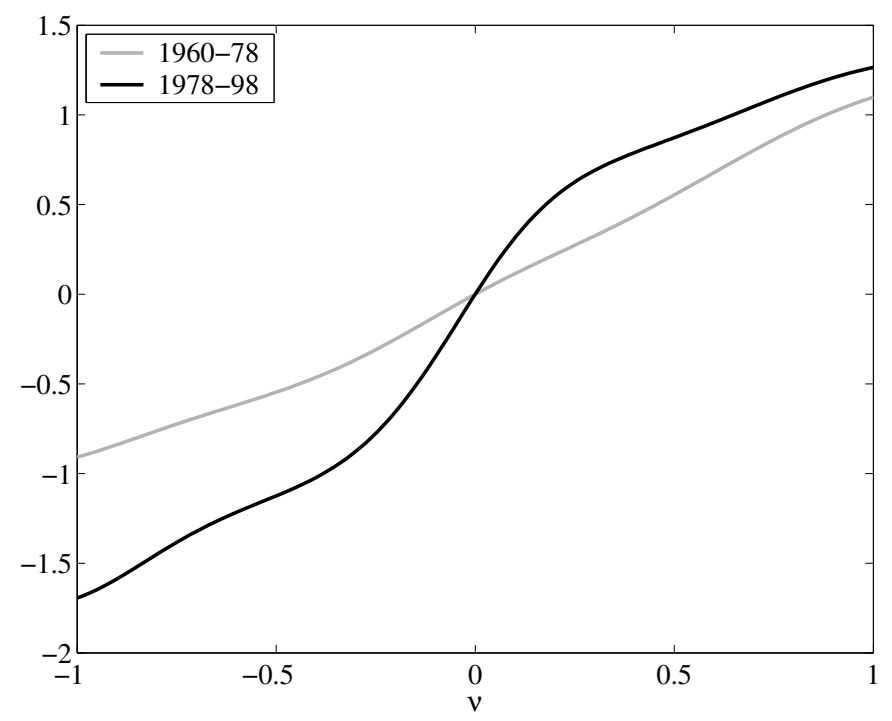

model prediction. ${ }^{28}$

\subsection{Do the More Open Economies Behave Differently?}

Up to now, our empirical approach has been to highlight changes in the growth process that have arisen during a period where there has been a substantial increase in international trade. However, in itself, the finding of increased sensitivity of $\widehat{y}_{i}$ with respect to $\widehat{\nu}_{i}$ over time does not necessarily imply that the change in the growth process is related to emergence of new trading opportunities. It only supports the theoretical mechanism presented in the model. One way to address this issue more directly is to look at whether the increased sensitivity of $\widehat{y}_{i}$ with respect to $\widehat{\nu}_{i}$ is more pronounced among countries most likely affected by the process of globalization. To this end, we consider two splits of the data. In the first case, we examine what happens among the initially more open countries versus the less open countries The idea behind this split is that the initially more open countries are the ones that can take advantage of new trading opportunities. Second, we examine what happens among countries which experienced the most increase trade between the two period. This second exercise can be thought as a robustness check on the first exercise since, if it is the more initially more open countries which take most advantage of the new trading opportunities, then we should expect the increased sensitivity of of $\widehat{y}_{i}$ with respect to $\widehat{\nu}_{i}$ to also be most apparent among the set of countries experiencing the most growth in trade.

In columns (1) and (2) of Table 5, we first report estimates of Equation (15) where we include

\footnotetext{
${ }^{28}$ Since we only have 75 observations in each subperiod, we recognize the need to be somewhat cautious with results based on non-parametric estimation with such small samples.
} 
as additional regressor the initial degree of openness of a country as measured by the ratio of export-plus-imports to GDP over the 1960-78 period. ${ }^{29}$ These results are reported in the first group of columns (openness). ${ }^{30}$ As can be seen, the inclusion of this openness measure does not affect our previous findings, as $\widehat{\nu}$ is found to approximately double between the period 1960-78 to 1978-98. We then estimate Equation (15) after dividing our sample into two sub-samples

Table 5: Openness

\begin{tabular}{|c|c|c|c|c|c|c|c|c|c|c|}
\hline & \multirow[b]{2}{*}{$\begin{array}{c}60-78 \\
(1)\end{array}$} & \multirow[b]{2}{*}{$\begin{array}{c}78-98 \\
(2)\end{array}$} & \multicolumn{2}{|c|}{$\mathrm{OP}<\operatorname{med}(\mathrm{OP})$} & \multicolumn{2}{|c|}{$\mathrm{OP} \geqslant \operatorname{med}(\mathrm{OP})$} & \multicolumn{2}{|c|}{$\Delta \mathrm{OP}<\operatorname{med}(\Delta \mathrm{OP})$} & \multicolumn{2}{|c|}{$\Delta \mathrm{OP} \geqslant \operatorname{med}(\Delta \mathrm{OP})$} \\
\hline & & & $\begin{array}{c}60-78 \\
(3)\end{array}$ & $\begin{array}{c}78-98 \\
(4)\end{array}$ & $\begin{array}{c}60-78 \\
(5)\end{array}$ & $\begin{array}{c}78-98 \\
(6)\end{array}$ & $\begin{array}{c}60-78 \\
(7)\end{array}$ & $\begin{array}{c}78-98 \\
(8)\end{array}$ & $\begin{array}{c}60-78 \\
(9)\end{array}$ & $\begin{array}{c}78-98 \\
(10)\end{array}$ \\
\hline \multirow[t]{2}{*}{ Cst. } & -0.008 & -0.017 & 0.001 & -0.015 & -0.005 & -0.011 & -0.001 & -0.017 & 0.003 & -0.006 \\
\hline & $(0.004)$ & $(0.004)$ & $(0.005)$ & $(0.004)$ & $(0.006)$ & $(0.004)$ & $(0.005)$ & $(0.003)$ & $(0.007)$ & $(0.005)$ \\
\hline \multirow[t]{2}{*}{$\widehat{y}_{0}$} & -0.012 & -0.014 & -0.009 & -0.014 & -0.015 & -0.013 & -0.008 & -0.015 & -0.010 & -0.009 \\
\hline & $(0.003)$ & $(0.003)$ & $(0.003)$ & $(0.003)$ & $(0.005)$ & $(0.005)$ & $(0.004)$ & $(0.004)$ & $(0.004)$ & $(0.003)$ \\
\hline \multirow[t]{2}{*}{$\widehat{\nu}$} & 0.012 & 0.028 & 0.019 & 0.020 & 0.006 & 0.037 & 0.012 & 0.023 & 0.006 & 0.028 \\
\hline & $(0.004)$ & $(0.005)$ & $(0.005)$ & $(0.005)$ & $(0.007)$ & $(0.007)$ & $(0.006)$ & $(0.007)$ & $(0.007)$ & $(0.007)$ \\
\hline \multirow[t]{2}{*}{ OP } & 0.009 & 0.007 & - & - & - & - & - & - & - & - \\
\hline & $(0.004)$ & $(0.004)$ & & & & & & & & \\
\hline $\mathcal{Q}($ Total $)$ & 7.595 & {$[0.055]$} & 1.285 & {$[0.526]$} & 27.051 & {$[0.000]$} & 1.156 & {$[0.561]$} & 7.214 & {$[0.027]$} \\
\hline $\mathcal{Q}\left(\widehat{y}_{0}\right)$ & 0.283 & {$[0.595]$} & 0.996 & {$[0.318]$} & 0.105 & {$[0.746]$} & 0.831 & {$[0.362]$} & 0.029 & {$[0.865]$} \\
\hline $\mathcal{Q}(\widehat{\nu})$ & 5.740 & {$[0.017]$} & 0.015 & {$[0.903]$} & 11.287 & {$[0.001]$} & 1.150 & {$[0.284]$} & 4.515 & [0.034] \\
\hline $\mathcal{Q}(\mathrm{OP})$ & 0.119 & {$[0.730]$} & - & - & - & - & - & - & - & - \\
\hline
\end{tabular}

Note: Standard errors in parenthesis, p-values in brackets. All regressions are estimated by instrumental variables, where the set of instrument the average (c/y) over the sub-sample, the average growth rate of population over the 15 first periods of the sub-sample.

according to their degree of initial openness (OP). The first sub-sample consists of the set of countries with a below median value of openness over the first sub-period, while the second sample corresponds to those with an above median value of openness over the same period. We perform this dichotomous procedure since it allows a direct reference to the observations presented in Section 1.1, later we will allow the openness variable to interact in a continuous fashion with $\widehat{\nu}_{i}$. Recall that in Section 1.1, we documented that it was only among the more open economies that an increase in dispersion was observed over the period 1978-98. Given this observation, it is interesting to ask whether it is also only among this group that we see an increase in the sensitivity of $\widehat{y}_{i}$ with respect to $\widehat{\nu}_{i}$. The results from estimating Equation (15) on these two sub-samples is reported in the second group of columns (Columns (3)-(6)) of Table 5. As can be clearly seen, it is only in the sample of more open economies where we observed

\footnotetext{
${ }^{29}$ As in Section 1.1, we calculate this measure of openness using the average value of export-plus-imports to GDP for the period 1960-78. Results are robust to calculating this average over the entire period or each sub-period.

${ }^{30}$ All the results in this table are derived from instrumental variable estimation using the set IV1 of instruments. The results presented in section are robust to also including human capital variables as additional regressors.
} 
a significant increase in the coefficient $\widehat{\nu}_{i}$. In fact, the coefficient of $\widehat{\nu}_{i}$ is found to be almost perfectly stable for the less open economies (0.019 for the 1960-78 period, 0.020 for 1978-98) while exhibiting a substantial increase for the more open economies, as it increase from 0.006 for the period $1960-78$ to 0.037 for the period 1978-98.

Figure 6: Rolling regressions
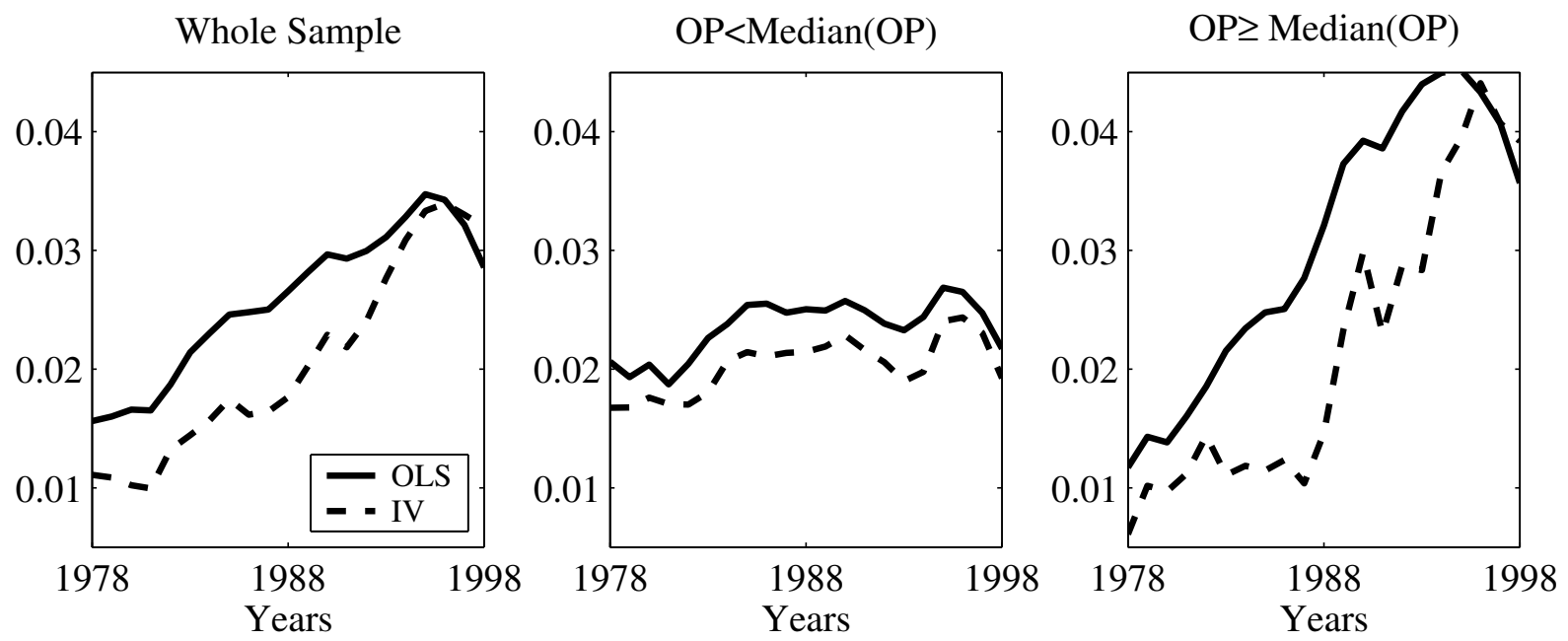

In order to visualize that change in the sensitivity of $\widehat{y}_{i}$ to $\widehat{\nu}_{i}$ over time for the whole sample as well as the two sub-sample of initially more open and more closed economies ( $\mathrm{OP}<$ median $(\mathrm{OP})$ ) and $(\mathrm{OP} \geqslant \operatorname{median}(\mathrm{OP}))$, we ran a set of 20 rolling regressions over 19 years windows starting with the period 1960-78. Figure 6 reports the estimated coefficients on $\widehat{\nu}_{i}$ obtained from rolling regressions of Equation (15) over the whole sample and these two sub-samples, with each variable measured in relation to the given window. ${ }^{31}$ In each case, we plot rolling OLS and IV estimates where the instrument set is IV1.

What is clear from Figure 6 is that the increase in the sensitivity of $\widehat{y}_{i}$ to $\widehat{\nu}_{i}$ appears almost exclusively among the open countries. This is in line with the spirit of our model since it is the most open economies that are the ones most likely to take advantage of new trading opportunities such as those associated with trade in intermediates. In effect, for the countries most open to international trade, the effects of $\widehat{\nu}_{i}$ appear to have tripled. One of the attractive features of this figure is that it clearly illustrates that the phenomenon we have been documenting regarding the increased sensitivity of $\widehat{y}_{i}$ to $\widehat{\nu}_{i}$ is not fragile to our cutting of the sample exactly between 1960-78 versus 1978-98. Instead, what we see is that the coefficient on $\widehat{\nu}_{i}$ increases continuously as we include more in more observation in the period 1978-98 versus the period 1960-78.

\footnotetext{
${ }^{31}$ We estimate a series of regressions with the first regression using observations 1 through 19, the second regression using observations 2 through 20, etc... The abscissae indicating the last year of the sub-sample. We also explored window sizes between 15 to 25 years, all of which led to similar conclusions. In Beaudry and Collard [2002] this approach was used to examine changes in the growth process among OECD country's.
} 
To further explore the relationship between openness and accumulation, in Columns (7)-(10) of Table 5, we report estimates of Equation (15) based on an alternative break of the sample. In particular, we now define two sub-samples according to observed increases in international trade between the period $1960-78$ and $1978-98$. The first sample $(\Delta \mathrm{OP}<\operatorname{median}(\Delta \mathrm{OP}))$ is the set of countries with below median change in international trade, while second sample $(\Delta \mathrm{OP} \geqslant$ median $(\Delta(\mathrm{OP}))$ is comprised of countries with above median increase. When we split the sample according to the change in the degree of openness, the results again provide support to the idea of an interaction between openness and accumulation in the period 1978-98. In fact, we see that the increased sensitivity of $\widehat{y}_{i}$ to $\widehat{\nu}_{i}$ is much more prevalent among the countries which above median change in international trade than with countries with below median growth. In effect for the set with $\Delta O P \geqslant$ median $(\Delta \mathrm{OP})$ the coefficient on $\widehat{\nu}_{i}$ increased from 0.006 to 0.028 , while for the set of countries with $\triangle O P \geqslant \operatorname{median}(\Delta)$ the same coefficient only increased from 0.012 to 0.023 (which is not a statistically significant change). In order to verify once again that these results are not driven by the omission of important country characteristics, in Appendix D we report estimates for both sample splits underlying Table 5 allowing for country fixed effects.

Table 6: Openness in Interaction

\begin{tabular}{lcccccccc}
\hline \hline & \multicolumn{2}{c}{ OLS } & \multicolumn{2}{c}{ IV $^{\star}$} & \multicolumn{2}{c}{ OLS } & \multicolumn{3}{c}{ IV $^{\star}$} \\
& $60-78$ & $78-98$ & $60-78$ & $78-98$ & $60-78$ & $78-98$ & $60-78$ & $78-98$ \\
& $(1)$ & $(2)$ & $(3)$ & $(4)$ & $(5)$ & $(6)$ & $(7)$ & $(8)$ \\
\hline Cst. & -0.004 & -0.013 & -0.000 & -0.013 & -0.004 & -0.013 & -0.000 & -0.015 \\
& $(0.003)$ & $(0.003)$ & $(0.004)$ & $(0.003)$ & $(0.003)$ & $(0.003)$ & $(0.004)$ & $(0.003)$ \\
$y_{0}$ & -0.013 & -0.013 & -0.010 & -0.014 & -0.013 & -0.014 & -0.010 & -0.016 \\
& $(0.002)$ & $(0.002)$ & $(0.003)$ & $(0.002)$ & $(0.002)$ & $(0.002)$ & $(0.003)$ & $(0.002)$ \\
$\nu$ & 0.020 & 0.019 & 0.013 & 0.017 & 0.018 & 0.025 & 0.016 & 0.024 \\
& $(0.005)$ & $(0.005)$ & $(0.008)$ & $(0.007)$ & $(0.003)$ & $(0.004)$ & $(0.005)$ & $(0.004)$ \\
OP $\times \nu$ & -0.007 & 0.017 & -0.005 & 0.024 & - & - & - & - \\
& $(0.007)$ & $(0.007)$ & $(0.011)$ & $(0.009)$ & & & & \\
$\Delta \mathrm{OP} \times \nu$ & - & - & - & - & -0.016 & 0.031 & -0.032 & 0.060 \\
& & & & & $(0.011)$ & $(0.014)$ & $(0.016)$ & $0.021)$ \\
& 0.37 & 0.55 & - & - & 0.38 & 0.54 & - & - \\
\hline $\mathcal{Q}(\mathrm{Total})$ & 20.982 & {$[0.000]$} & 26.945 & {$[0.000]$} & 20.484 & {$[0.000]$} & 38.023 & {$[0.000]$} \\
$\mathcal{Q}\left(y_{0}\right)$ & 0.002 & {$[0.961]$} & 1.517 & {$[0.218]$} & 0.036 & {$[0.849]$} & 3.024 & {$[0.082]$} \\
$\mathcal{Q}(\nu)$ & 0.035 & {$[0.852]$} & 0.167 & {$[0.682]$} & 1.626 & {$[0.202]$} & 2.022 & {$[0.155]$} \\
$\mathcal{Q}(\mathrm{x} \times \nu)$ & 7.668 & {$[0.006]$} & 3.940 & {$[0.047]$} & 7.351 & {$[0.007]$} & 13.715 & {$[0.000]$} \\
\hline \hline
\end{tabular}

Note: Standard errors in parenthesis, p-values in brackets. The set of istrumental variables $\mathrm{IV}^{\star}$ is discussed in the body text.

In Table 6 , we allow $\widehat{\nu}_{i}$ to interact continuously with, first with the countries average degree or openness (results in Columns (1)-(4) of the table) or second with the change in openness between periods 1960-78 versus 1978-98 (results Columns (5)-(8)). The difference between the sets of 
estimates under the heading OLS and $\mathrm{IV}^{\star}$ is that in the first case, the estimates are derived without instrumenting neither $\widehat{\nu}_{i}$ nor the openness variable. In contrast, for the results under the heading $\mathrm{IV}^{\star}$, both the openness variable and $\widehat{\nu}_{i}$ are instrumented. The set of instruments used to instrument openness correspond to the instruments used in Hall and Jones [1999]. ${ }^{32}$ In all these sets of estimates, we see that there is a significant positive interaction between openness and $\widehat{\nu}_{i}$ in the second period but not in the first, which suggests that over the period 1978-98 the more a country was open (or the more it experienced growth in trade), the more it experienced a high return to being a capital intensive country. Moreover, we can see from the estimates in the first two columns, that for a fictitious entirely closed economy (i.e., a country with zero imports plus exports ${ }^{33}$ ) the returns to capital is almost unchanged between the two periods, which is consistent with the idea that it is only if a country was open to international trade that it could derive greater benefits by accumulating capital since it could thereby attract high wage jobs. ${ }^{34}$

\subsection{What Drives Changes in the Distribution of Output-per-Worker?}

The above regression results indicate a significant increase in the sensitivity of $\widehat{y}_{i}$ with respect to $\widehat{\nu}_{i}$ between the period 1960-78 versus the period 1978-98. However, the fact that the increase is statistically significant does not imply that it is economically major in the sense of being an important factor behind the increase dispersion of income across countries over the period 1978 to 1998. In effect, a central claim of this paper is not just that the data provide evidence in support of the mechanism emphasized in the model but that in addition this mechanism is a key element in the observed changes in the cross-country distribution of income.

In order to explore the extent to which the observed increase sensitivity of $\widehat{y}_{i}$ with respect to $\widehat{\nu}_{i}$ may be relevant for understanding the observed change in the cross-country distribution of output-per-worker from 1978 to 1998, in Figure 7, we overlay the actual distribution observed in 1998 and 1978 with a set of counterfactual distributions designed to control for different factors. The procedure we take for building counterfactuals is common in the literature, especially in the wage inequality literature (see e.g. Juhn, Murphy and Pierce [1993] among others). To understand the construction of these counterfactuals, it is necessary to first recognize that $\widehat{y}_{98}$

\footnotetext{
${ }^{32}$ The of instrument is the average (c/y) over the sub-sample, the average growth rate of population over the 15 first periods of the sub-sample and the beginning of period investment-to-output ratio. The openness variable is instrumented by the fraction of the population speaking one of the 5 European languages, the same fraction for English, the Frankel and Romer geographical based trade instrument and the distance from equator. Finally, we have interactions between the instruments for accumulation and those for openness.

${ }^{33}$ The openness variable is not expressed as a deviation from the US level as to allow as easier interpretation.

${ }^{34} \mathrm{We}$ also estimated the relations of Table 6 allowing for country fixed effects. These results are available from the authors upon request. In this case, we found significant and positive increases in the interaction between $\widehat{\nu}_{i}$ and the openness measure (whether it be its level or it difference). Moreover, the magnitude of the change was very similar to that observed in Table 6 . In this sense the results of Table 6 are robust to allowing for country fixed effects. However, when we allow for fixed effects, for the period 1960-78, we found a significant negative link between openness and $\widehat{\nu}_{i}$ for which we have no clear interpretation.
} 
can be written as below as the sum of $\widehat{y}_{78}$, the estimated growth process between 1978 and 1998 , and the associated residuals:

$$
\widehat{y}_{98}=\widehat{y}_{78}+\eta_{0}^{78-98}+\eta_{y}^{78-98} y_{i}^{78}+\eta_{\widehat{\nu}_{i}}^{78-98} \widehat{\nu}_{i}^{78-98}+\varepsilon_{i}^{78-98}
$$

where $\eta_{0}^{78-98}, \eta_{y}^{78-98}$ and $\eta_{\widehat{\nu}_{i}}^{78-98}$ correspond respectively to the constant, the coefficient on $\widehat{y}_{0}$ and the coefficient on $\widehat{\nu}_{i}$ derived from the estimation of the growth process between 1978-98, while $\varepsilon_{i}^{78-98}$ represents the residuals from the regression. Given this framework, it is possible to build counterfactuals for $\widehat{y}_{98}$ by replacing different terms in Equation (17). This hinges on a preliminary estimation of the growth process. Henceforth, all the counterfactuals are based on the coefficient derived from estimating Equation (15) by instrumental variables using the set IV1 of instruments. In panel (a) of Figure 7, we build a distribution for $\widehat{y}_{98}$ by assuming a

Figure 7: Counterfactual Distributions

(a) Coefficients (v)

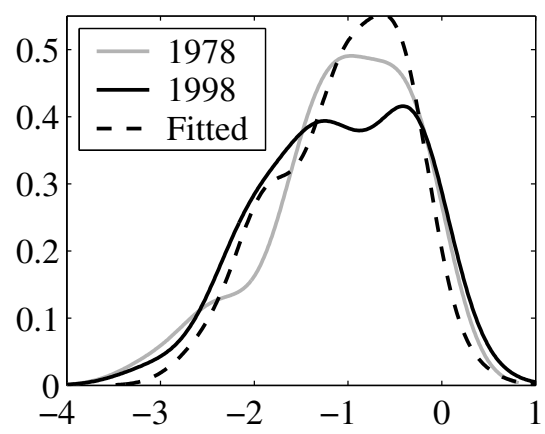

(c) Convergence

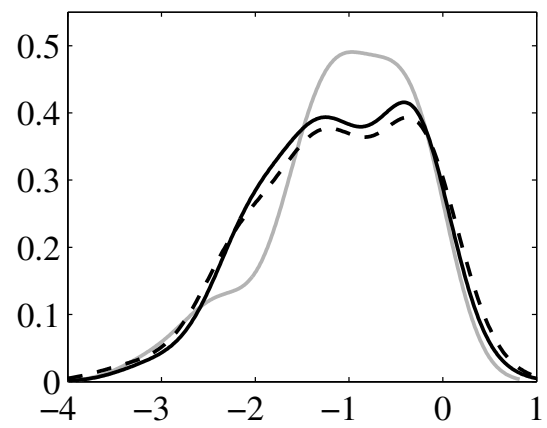

(b) Distribution (v)

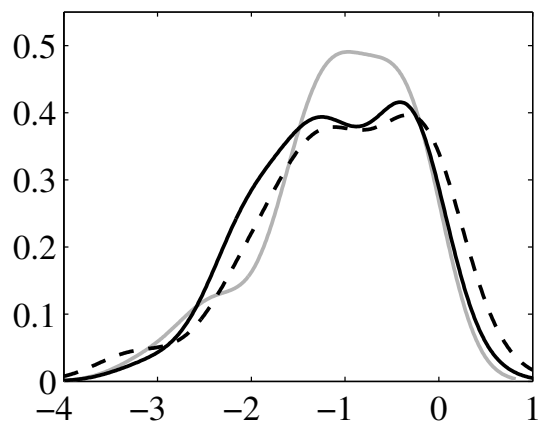

(d) Unobservables

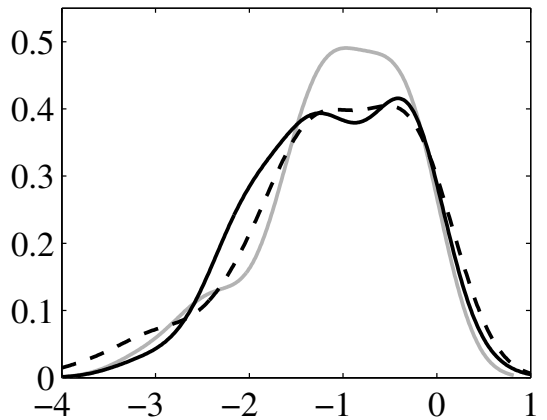

counterfactual growth process for the period 1978-98 whereby the coefficient on $\widehat{\nu}$ in the growth process is assumed to take on the value observed over the period 1960-78 instead of its actual value. In other words, to build the counterfactual that controls for the increase sensitivity of $\widehat{y}_{i}$ with respect to $\widehat{\nu}_{i}$, in Equation (17) we replace $\eta_{\widehat{\nu}_{i}}^{78-98}$ by $\eta_{\widehat{\nu}_{i}}^{60-78}$. This can be understood, in light of the framework developed in Section 1.2, as controlling the distribution of output-perworker for a change in $g_{t}(\cdot)$. We can see from the figure, the estimated changes in $\eta_{\widehat{\nu}_{i}}$ appears to account for a substantial amount of the change in the shape of the distribution between 1978 
and 1998. In fact, we see that much more mass would be concentrated around the mode of the distribution if the coefficient on $\eta_{\widehat{\nu}_{i}}$ had not increased.

In order to place this result in perspective, it is helpful to compare it with alternative counterfactuals. To this end, we build three other counterfactuals. ${ }^{35}$ In panel (b) of Figure 7, we present a counterfactual where we replace $\widehat{\nu}_{78-98}$ with $\widehat{\nu}_{60-78}$, that is, we build a counterfactual that controls for changes in the distribution of $\widehat{\nu}$ between the two periods. This counterfactual is particularly relevant for evaluating the importance of alternative models which emphasize changes in the distribution of $\widehat{\nu}$. Controlling for this effect amounts to control for a change in the function $h_{t}(\cdot)$ described in Section 1.2. In other words, this counterfactual controls for any effect of globalization on the distribution on the long-run propensity to accumulate capital. Recall that this channel of change is an important component of the model in Ventura [1997]. As can be seen in the figure, the change in the distribution of $\widehat{\nu}$ over the two periods cannot account for much of the change in shape of the distribution between 1978 and 1998 since the resulting counterfactual distribution for 1998 is almost identical to the actual distribution in 1998.

In the two remaining panels of the figure, we present a counterfactual where, in panel (c), we replace $\eta_{y}^{78-98} y_{i}^{78}$ with $\eta_{y}^{60-78} y_{i}^{60}$ and, in panel (d), we present a counterfactual based on replacing $\varepsilon_{i}^{78-98}$ by $\varepsilon_{i}^{60-78}$. The first of these two counterfactuals is meant to control for any change in the dependence of growth on initial conditions, while the second is meant to capture the potential effects of omitted variables. Again, in both case we see that the counterfactuals explain little of the change in shape of the distribution between 1978-98.

Instead of a graphical representation of the explanatory power of these counterfactuals, in Table 7 we report a set quantitative measures. In the first column of Table 7, we reports for comparison the set of changes in percentiles observed over the period 1978-98 discussed in Section 1.1. In the next three columns we report a set of changes in percentiles that would have been observed under three counterfactuals. In the first counterfactual (which corresponds to the counterfactual given in the first panel of Figure 7), we report the change in percentiles that would have been observed if the coefficient on $\widehat{\nu}_{i}$ would have maintained its 1960-78 value of 0.011 over the period 1978-98 instead of having increased to 0.030. As can be seen, many of the entries in the second column of Table 7 are very close to zero or even negative suggesting that if the sensitivity of $\widehat{y}_{i}$ to $\widehat{\nu}_{i}$ had not increased we would have seen essentially no changes or even minor decreases in many percentile differences. The only exceptions are for the interquartile range and the 10-90 percentile difference. For the interquartile range, the counterfactual can explain about half of the increase observed over the period 1978-98, that is, this counterfactual suggests that the increase in the interquartile range would have been only of $18.7 \%$ instead of the $37.6 \%$ observed in the

\footnotetext{
${ }^{35}$ Greater details on the construction of the counterfactual distribution can be found in BCG.
} 
Table 7: Changes in Percentiles

\begin{tabular}{ccccc}
\hline \hline perc. & $98-78$ & $\eta_{\nu}$ & $\Delta \mu^{\nu}(\cdot)$ & $\eta_{0} y_{0}$ \\
\hline $10-90$ & -0.162 & -0.430 & 0.055 & 0.052 \\
$15-85$ & 0.444 & 0.093 & 0.454 & 0.539 \\
$20-80$ & 0.314 & 0.063 & 0.219 & 0.442 \\
$25-75$ & 0.376 & 0.187 & 0.330 & 0.398 \\
$30-70$ & 0.216 & -0.059 & 0.309 & 0.308 \\
$35-65$ & 0.224 & -0.115 & 0.259 & 0.286 \\
$40-60$ & 0.103 & -0.007 & 0.155 & 0.090 \\
$45-55$ & 0.038 & -0.024 & 0.090 & -0.006 \\
\hline \hline
\end{tabular}

data. In the case of the 10-90 difference, the counterfactual actually over-predicts the narrowing in the distribution relative to what was observed. Hence, we believe that this counterfactual supports the notion that the rise in the sensitivity of $\widehat{y}_{i}$ to $\widehat{\nu}_{i}$ can explain over $50 \%$ of the change in distribution observed over the period 1978-98.

The third column reports the counterfactual percentile changes for the case where the distribution of $\widehat{\nu}_{i}$ in the period 1978-98 is replaced by the distribution observed in the period 1960-78. In this case we see that the counterfactual percentile changes are of very similar order of magnitude to that actually observed over the period 1978-98. For example, in the case of the interquartile range, this counterfactual can only explain about one twelfth of the observed increase, and in many cases it generates a greater widening of this distribution under the counterfactual that what was observed. This confirms the visual impression given in Figure 7 that the change in the distribution of $\widehat{\nu}_{i}$ between the two periods is at best a minor element in explaining the observed change in the distribution.

Finally, in the last column, we report the counterfactual percentile changes associated with having the dependence of growth on initial condition have the same force over the period 197898 than that observed over the period 1960-78. The interest in this counterfactual lies in offering an evaluation of the idea that the change in the distribution observed over 1978-98 may be due to process whereby the richer were getting richer and the poor poorer precisely because of a change in the dependence on initial conditions. As in the third column, we see that very little of the observed changes in percentiles appears to be accounted for by such a mechanism. ${ }^{36} \mathrm{In}$

\footnotetext{
${ }^{36}$ This result may be criticized on the ground that we only allow initial conditions to enter in a linear fashion. Therefore, we verified the robustness of this finding against allowing the country's initial level of output-perworker to enter nonlinearly in the growth regressions. Interestingly, when we do not include $\widehat{\nu}_{i}$ in the regression, we find some support for divergence for middle income countries in the second period, that is, growth is found to be slightly positively related to its initial level for middle income countries over the period 1978-98. However, once we include $\widehat{\nu}_{i}$, we consistently find that growth is negatively related to initial conditions. This supports the notion that the data may look at first pass as if increase dispersion is being driven by the rich getting richer and the poor getting poorer, while when one controls for $\widehat{\nu}_{i}$, one finds that divergence appears driven primarily by an
} 
fact, if we focus on the interquartile range, we see that this counterfactual actual implies an increase larger than what was observed. Overall, these decomposition exercises suggest that the observed changes in the coefficient on $\widehat{\nu}_{i}$ is of the right order of magnitude to explain much of the observed change in the distribution, thereby providing support that the relevance of the mechanism emphasized in the model. In contrast, these exercises give little support to the idea that the change in the distribution of $\widehat{\nu}_{i}$ nor a change in the importance of initial conditions were central over this period once we control for changes in the importance of $\widehat{\nu}_{i}$.

\subsection{Do High $\nu$ Countries Concentrate Employment in Capital Intensive Goods?}

In this section we examine whether, as implied by the model, countries with high values of $\nu_{i}$ experience a greater concentration of employment in high capital intensive jobs during a period of globalization. In order to make headway on this issue, we gathered data on employment in manufacturing and non-manufacturing from the LABORSTA database. ${ }^{37}$ This data source allows us to have a consistent series of employment data for this sectorial breakdown for 42 countries over the period 1978-1998. Regrettably, these data do not go back to 1960 as with our output data. Nonetheless, they provide sufficient information to examine whether over the period 197898 countries with higher values of $\nu_{i}$ experience a higher growth in the ratio of manufacturing employment to total employment relative to countries with low values of $\nu_{i}$. Taking for given that the manufacturing sector tends to be more capital intensive, we examine here whether employment patterns conform to the predictions of the model. Let us nevertheless stress that we view this exercise as a very rough way of looking at the implication of the model in term of employment patterns since we believe that the forces emphasized by the model are at least as important within industrial sectors as between sectors. Column (1) of Table 8 reports results from regressing the change in the ratio of manufacturing employment to total employment on its initial value, denoted $\operatorname{man}_{0}$ and on $\widehat{\nu}$. This regression is done by OLS, while in the second column we estimate the relationship by instrumental variables using IV1 as instruments. As can be seen, the effect of $\widehat{\nu}_{i}$ is found to be positive and quite significant in both the OLS or IV regression. In the last two columns of the table, we divide the sample into the more open and more close economies as we had done previously based on a countries ratio of imports plus exports to GDP. The interesting finding in Table 8 is that all the results are consistent with our previous findings in that open countries with high values of $\widehat{\nu}_{i}$ appear to have experienced faster growth in the manufacturing employment during the period 1978-98 while simultaneously experiencing high growth in output-per-worker. ${ }^{38}$ Together we believe that these observations further support

increase in the return to $\widehat{\nu}_{i}$ not a change in dependance on initial conditions.

${ }^{37}$ This database is available from the ILO website: http://www.ilo.org.

${ }^{38}$ In BCG we also document that the countries with high values of $\widehat{\nu}_{i}$ experience particularly high growth in TFP over the period 1978-98. 
Table 8: Effect on the changes in the labor share in Manufacturing (1978-1998)

\begin{tabular}{|c|c|c|c|c|c|}
\hline & $\begin{array}{c}\text { OLS } \\
(1)\end{array}$ & $\begin{array}{c}\text { IV1 } \\
(2)\end{array}$ & $\begin{array}{c}\text { IV1 } \\
(3)\end{array}$ & $\begin{array}{c}\mathrm{OP}<\operatorname{med}(\mathrm{OP}) \\
\mathrm{IV} 1 \\
(4)\end{array}$ & $\begin{array}{c}\mathrm{OP} \geqslant \operatorname{med}(\mathrm{OP}) \\
\mathrm{IV} 1 \\
(5)\end{array}$ \\
\hline \multirow[t]{2}{*}{ Cst. } & 0.006 & 0.006 & 0.005 & 0.003 & 0.009 \\
\hline & $(0.001)$ & $(0.001)$ & $(0.001)$ & $(0.001)$ & $(0.002)$ \\
\hline \multirow[t]{2}{*}{$\operatorname{man}_{0}$} & -0.040 & -0.042 & -0.039 & -0.028 & -0.058 \\
\hline & $(0.004)$ & $(0.005)$ & $(0.005)$ & $(0.004)$ & $(0.009)$ \\
\hline \multirow[t]{2}{*}{$\widehat{\nu}$} & 0.012 & 0.016 & 0.027 & 0.007 & 0.035 \\
\hline & $(0.006)$ & $(0.007)$ & $(0.011)$ & $(0.006)$ & $(0.015)$ \\
\hline$\widehat{H}$ & - & - & $\begin{array}{c}-0.002 \\
(0.001)\end{array}$ & - & - \\
\hline$R^{2}$ & 0.70 & - & - & - & - \\
\hline
\end{tabular}

Note: Standard errors in parenthesis, p-values in brackets. 42 observations. 39 observations when education is introduced. The coefficient infront of $\nu$ is multiplied by 100 for easier presentation.

the broad picture of economic change due to globalization implied by the model. ${ }^{39}$

\section{Concluding Remarks}

In many countries, workers and worker movements are observed to have strong reservations about free trade and globalization due to the risk of loosing the best paying jobs to their trading partners. However, in a world economy, not all countries can loose "good jobs", some have to be on the receiving end. In this paper we have presented a model that captures the notion that freer trade may cause a redistribution of rent paying jobs across countries. The two most useful features of the model are (i) it highlights who should be the winners and who are the losers in such a process, and (ii) it indicates how to approach the data to evaluate such a possibility. In particular, it indicates that the winners should be countries which most favor capital accumulation, and it indicates how to use cross-countries growth regression to evaluate this claim. After looking at changes in growth processes, decomposition exercises and employment patterns, we conclude that the data provide considerable support to the idea that globalization, through its effect on the redistribution of rent paying jobs, may have been a central element in the recent reshaping of the cross-country distribution of income.

\footnotetext{
${ }^{39}$ Note that the observation that high $\widehat{\nu}$ countries experience high growth in manufacturing employment during a period of globalization does not help differentiate the model regarding whether $x$ is greater or equal to zero since the model has the same implication on this front regardless of the value of $x$.
} 


\section{References}

Abowd, J.M., F. Kramarz, and D.N. Margolis, High Wage Workers and High Wage Firms, Econometrica, 1999, 67 (2), 251-333.

Arai, M., Wages, Profits and Capital Intensity: Evidence from Matched Worker-Firm Data, Journal of Labor Economics, 2003, 21 (3), 593-618.

Barro, R. and J.W. Lee, International Comparisons of Educational Attainment, Journal of Monetary Economics, 1993, 32 (3), 363-394.

Beaudry, P. and F. Collard, Why has the Employment-Productivity Tradeoff among Industrialized Countries been so Strong?, Working Paper 8754, NBER 2002.

_ _ _ and D. Green, Decomposing the Twin Peaks: A Study of the Changing World Distribution of output per worker, Working Paper 9240, NBER 2002.

Dickens, W.T. and L.F. Katz, Inter-Industry Wage Differences and Industry Characteristics, in K. Lang and J.S. Leonard, editors, Unemployment and the Structure of Labor Markets, Oxford: Basil Blackwell, 1987.

Gibbons, R. and L.F. Katz, Does Unobserved Ability Explain Inter-Industry Wage Differentials?, Review of Economic Studies, 1992, 59, 515-536.

Hall, R.E. and C.I. Jones, Why do Some Countries Produce so Much More Output per Worker than Others?, Quarterly Journal of Economics, 1999, 114 (1), 83-116.

Jones, C.I., On the Evolution of the World Income Distribution, Journal of Economic Perspectives, 1997, 11 (3), 19-36.

Juhn, C., K.M. Murphy, and B. Pierce, Wage Inequality and the Rise in Returns to Skill, Journal of Political Economy, 1993, 101 (3), 410-442.

Kremer, M., A. Onatski, and J.H. Stock, Searching for Prosperity, Working Paper 8250, NBER 2001.

Krueger, A.B. and L.H. Summers, Efficiency Wages and the Inter-Industry Wage Structure, Econometrica, 1988, 56 (2), 259-293.

Mankiw, N.G., D. Romer, and D.N. Weil, A Contribution to the Empirics of Economic Growth, Quarterly Journal of Economics, 1992, 107 (2), 407-437.

Quah, D., Empirical Cross-Section Dynamics in Economic Growth, European Economic Review, 1993, $37(2 / 3), 426-434$. 
_ _ Empirics for Growth and Distribution: Polarization, Stratification, and Convergence Clubs, Journal of Economic Growth, 1997, 2 (1), 27-59.

Solow, R.M., A Contribution to the Theory of Economic Growth, Quarterly Journal of Economics, 1956, 70 (1), 65-94.

Ventura, J., Growth and Interdependence, Quarterly Journal of Economics, 1997, pp. 57-84. 


\section{APPENDIX}

\section{A Allowing for International Capital Flows}

In this section, we discuss the implications of relaxing the assumption of no capital mobility in the model. More precisely, we document the extent to which our main results are robustness to allowing for trade on financial capital markets.

Let us first consider the case of a perfectly frictionless international financial markets. In this case, the returns to capital are equalized across countries and the location of capital is independent of the countries propensity to save. Therefore, in the absence of trade in intermediate goods - and in the absence of differences in $\Omega_{i}$ - the level of output-per-worker is identical across countries. In contrast, when trade in intermediate goods is allowed, the cross country distribution of output-per-worker is indeterminate since there are two mechanisms for equalizing the returns to capital across country: through trade or through international capital flow. Hence, in the extreme case of perfect international capital markets, the model has no clear predictions on how the opening up of trade will affect the cross-country distribution of output. This is a rather unsatisfactory result. In order to have a better sense of how our results can be extend in the presence of international capital flows, it is useful to consider the limiting behavior of a model with imperfections in international capital market.

To this end, let us consider the case where domestic firms face a risk premium on borrowing in the international market which is proportional to the country's debt-to-GDP ratio, and let us examine the outcome when this risk premium tends to zero. More precisely, let us assume that the cost of capital in country $i, q_{i}$, is equal to the cost of capital in the large reference economy (the US) plus a risk premium which is proportional to the country's debt to GDP ratio as given by

$$
q_{i}=q_{0}+\rho\left(\frac{k_{i}-a_{i}}{y_{i}}\right)
$$

where $\rho$ is the gradient of the risk premium, $a_{i}$ is the wealth-per-worker in country $i$ and hence $\left(k_{i}-a_{i}\right)$ is the amount of international debt-per-worker in country $i$. Through the accumulation equation, the wealth-per-worker along a steady growth path is given by $a_{i}=\nu_{i} y_{i}$ and therefore the determination of the domestic cost of capital can alternatively be written as

$$
q_{i}=q_{0}+\rho\left(\frac{k_{i}}{y_{i}}-\nu_{i}\right)
$$

Given this equation for the determination of cost of capital, a country level of capital-per-worker and output-per-worker is determined by equating the international cost of capital to the domestic return on capital. In the absence of international trade in intermediates, the limiting outcome as $\rho$ goes to zero will have all countries producing the same amount of output-per-worker since this is the only way the returns to capital can be equalized across countries. Hence, in this case - and assuming no differences in $\Omega_{i}$ - the cross country distribution of output-per-worker is concentrated at a single point.

If we now open up trade in intermediates, the determination of output-per-worker in country $i$ depends on $\nu_{i}$. In particular, if $\underline{\underline{\nu}} \leqslant \widehat{\nu}_{i} \leqslant \overline{\widehat{\nu}}$ then the determination of output-per-worker remains the same as in the absence international capital flows since the returns to capital are equalized (recall that within the incomplete specialization area, the social returns are constant). As a matter of fact, in the presence of international capital flows, it is easy to verify that in the limit as $\rho$ goes to zero, the determination of $\widehat{y}_{i}$ is given by

$$
\widehat{y}_{i}= \begin{cases}\log \left(\frac{(1-\alpha) \varphi+(1-\varphi) \frac{1-\beta}{1+x}}{1-\alpha}\right) & \text { if } \widehat{\nu}<\widehat{\underline{\nu}} \\ \log \left(\frac{(\beta-\alpha)\left(\varphi(1-\alpha)+(1-\varphi) \frac{1-\beta}{1+x}\right.}{\beta(1-\alpha)-\alpha \frac{1-\beta}{1+x}}\right)-\log \left(1-\frac{(\alpha \varphi+\beta(1-\varphi))\left(1-\alpha-\frac{1-\beta}{1+x}\right)}{\beta(1-\alpha)-\alpha \frac{1-\beta}{1+x}} \exp \left(\widehat{\nu}_{i}\right)\right) & \text { if } \widehat{\underline{\nu}} \leqslant \widehat{\nu} \leqslant \overline{\widehat{\nu}} \\ \log \left(\frac{(1-\alpha) \varphi+(1-\varphi) \frac{1-\beta}{1+x}}{\frac{1-\beta}{1+x}}\right) & \text { if } \widehat{\nu}>\widehat{\widehat{\nu}}\end{cases}
$$


As can be seen from the mapping between $\widehat{y}_{i}$ and $\widehat{\nu}_{i}$, in the limiting case where $\rho$ tends to zero, the opening up of trade in intermediates causes an increase in dispersion in output-per-worker since for countries with such that $\widehat{\nu}_{i} \in[\underline{\underline{v}}, \overline{\hat{\nu}}]$ output-per-worker is no longer equalized but instead becomes an increasing function of $\nu_{i}$. Furthermore, in addition to this increase in dispersion for the countries with $\widehat{\nu}_{i} \in[\underline{\widehat{\underline{\nu}}}, \widehat{\widehat{\nu}}]$, the countries with either $\widehat{\nu}_{i}<\underline{\widehat{\nu}}$ or $\widehat{\nu}_{i}>\overline{\widehat{\nu}}$ will bunch at two points in the distribution of $y_{i}$. Indeed, let us consider the case of a country with a low propensity to capital accumulation $\left(\widehat{\nu}_{i}<\underline{\widehat{\underline{v}}}\right)$. In the limiting case where $\rho$ tends to zero, the only way its return on capital can equalize the world return on capital is to accumulate up to the point its capital-output ratio reaches $\underline{\nu}$, such that its $\widehat{\nu}_{i}=\underline{\underline{\underline{\nu}}}$. This phenomenon likely gives rise to bi-modality. Therefore, the main results presented in the paper survive the introduction of international capital flows as long as the international capital market is not perfectly frictionless. ${ }^{40}$

\section{B Allowing for Endogenous World Prices}

In the main body of the paper, we have assumed that under free trade the world price for intermediate goods correspond to the autarky prices of these goods in the reference economy. Our defense for this assumption is that the reference economy used in our empirical work is the US economy and since the US economy is extremely large economically this may constitute a good approximation. However, it is clearly an approximation. Therefore in this section we discuss how our results must be modified and rephrased when this assumption is relaxed. It is rather easy to derive the mapping between $\widehat{\nu_{i}}$ and $\widehat{y_{i}}$ for the case where world prices under free trade do not correspond to the reference economy's autarky prices. To do so, let us denote by $\nu^{\star}$ the value of $\widehat{\nu}$ such that a country with $\widehat{\nu_{i}}=\nu^{\star}$ does not trade in equilibrium since the world prices of intermediates are equal to its autarky prices. Then, the mapping between $\widehat{\nu_{i}}$ and $\widehat{y_{i}}$ becomes

$$
\widehat{y}_{i}= \begin{cases}\frac{\alpha}{1-\alpha} \log \left(\frac{\alpha \varphi+\beta(1-\varphi)}{\alpha}\right)+\log \left(\frac{(1-\alpha) \varphi+(1-\varphi) \frac{1-\beta}{1+x}}{1-\alpha}\right)+\frac{\alpha}{1-\alpha}\left(\widehat{\nu}_{i}-\nu^{\star}\right) & \text { if }\left(\widehat{\nu}_{i}-\nu^{\star}\right)<\widehat{\underline{\nu}} \\ \log \left(\frac{(\beta-\alpha)\left(\varphi(1-\alpha)+(1-\varphi) \frac{1-\beta}{1+x}\right.}{\beta(1-\alpha)-\alpha \frac{1-\beta}{1+x}}\right)-\log \left(1-\frac{(\alpha \varphi+\beta(1-\varphi))\left(1-\alpha-\frac{1-\beta}{1+x}\right)}{\beta(1-\alpha)-\alpha \frac{1-\beta}{1+x}} \exp \left(\widehat{\nu}_{i}-\nu^{\star}\right)\right) & \text { if } \underline{\underline{\underline{\nu}}} \leqslant\left(\widehat{\nu}_{i}-\nu^{\star}\right) \leqslant \widehat{\widehat{\nu}} \\ \frac{\beta}{1-\beta} \log \left(\frac{\alpha \varphi+\beta(1-\varphi)}{\beta}\right)+\log \left(\frac{(1-\alpha) \varphi+(1-\varphi) \frac{1-\beta}{1+x}}{\frac{1-\beta}{1+x}}\right)+\frac{\beta}{1-\beta}\left(\widehat{\nu}_{i}-\nu^{\star}\right) & \text { if }\left(\widehat{\nu}_{i}-\nu^{\star}\right)>\overline{\widehat{\nu}}\end{cases}
$$

The previous equation makes it clear that the presence $\nu^{\star} \neq 0$ simply causes a translation of our original mapping between $\widehat{\nu_{i}}$ and $\widehat{y_{i}}$. However, the problem with this mapping is that we do not know the value of $\nu^{\star}$. Nonetheless, we can still make a conditional statement regarding how the opening of trade with affect the distribution of $\widehat{y_{i}}$. In particular, in this more general case, Corollary 1 should simply be rephrased as follows

If the distribution of $\widehat{\nu}_{i}$ is concentrated around $\nu^{\star}$, then the first order effect of free trade on the crosscountry distribution of $\widehat{y}_{i}$ is nil when $x=0$. In contrast, it leads to an increase in dispersion when $x>0$.

This extended version of Corollary 1 clarifies that our main results hinge on the notion that $\nu^{\star}$ be not too different from the mean of $\widehat{\nu_{i}} \cdot{ }^{41}$ In other words, the key condition for our results on the effect of free trade to hold is that the average capital-output ratio across countries must not be substantially different the capital-output ratio across the world. ${ }^{42}$

\footnotetext{
${ }^{40}$ In the presence of international capital flows, there are two distinct mechanisms which cause an increase in the dispersion of $y_{i}$. There is an increase due to increased dispersion of capital-output ratios and, if $x>0$, there is increased dispersion due to an increase in the return to capital. The model of Ventura [1997] is an alternative way of emphasizing the first mechanism, while the model present in the main body of this paper emphasizes the second mechanism. As the empirical section of the paper has shown, the data are more supportive of the second mechanism.

${ }^{41}$ In fact, the result can be strengthened slightly by noting that what is key for our results is that $\nu^{\star}$ not be substantially greater than the mean of $\widehat{\nu_{i}}$.

${ }^{42}$ Based on our calculation using the World Penn tables, this condition appears satisfied.
} 


\section{Composition of the sample}

Our sample of 75 countries consists of:

Argentina, Australia, Austria, Belgium, Bangladesh, Bolivia, Brazil, Barbados, Botswana, Canada, Switzerland, Chile, China, Colombia, Costa Rica, Cyprus, Denmark, Dominican Republic, Ecuador, Egypt, Spain, Finland, Fiji, France, United Kingdom, Greece, Guatemala, Guyana, Hong Kong, Honduras, Indonesia, India, Ireland, Iran, Iceland, Israel, Italy, Jamaica, Jordan, Japan, Republic of Korea, Sri Lanka, Lesotho, Luxembourg, Morocco, Mexico, Mozambique, Malaysia, Namibia, Nicaragua, Netherlands, Norway, Nepal, New Zealand, Pakistan, Panama, Peru, Philippines, Papua New Guinea, Portugal, Paraguay, Romania, Singapore, El Salvador, Sweden, Syria, Thailand, Trinidad and Tobago, Tunisia, Turkey, Taiwan, Uruguay, USA, Venezuela, South Africa,

\section{Fixed Effects}

Table 9: Fixed Effects

\begin{tabular}{lcccccccccc}
\hline \hline & \multicolumn{2}{c}{ OLS } & \multicolumn{2}{c}{ IV1 } & \multicolumn{2}{c}{ IV1 } & \multicolumn{2}{c}{ IV2 } & \multicolumn{3}{c}{ IV3 } \\
& $60-78$ & $78-98$ & $60-78$ & $78-98$ & $60-78$ & $78-98$ & $60-78$ & $78-98$ & $60-78$ & $78-98$ \\
\hline$\widehat{y}_{0}$ & -0.059 & -0.062 & -0.056 & -0.059 & -0.054 & -0.059 & -0.053 & -0.061 & -0.075 & -0.084 \\
& $(0.004)$ & $(0.005)$ & $(0.004)$ & $(0.005)$ & $(0.005)$ & $(0.006)$ & $(0.005)$ & $(0.006)$ & $(0.010)$ & $(0.012)$ \\
$\widehat{\nu}$ & 0.021 & 0.044 & 0.030 & 0.053 & 0.030 & 0.053 & 0.019 & 0.042 & 0.015 & 0.063 \\
& $(0.003)$ & $(0.004)$ & $(0.005)$ & $(0.007)$ & $(0.006)$ & $(0.009)$ & $(0.004)$ & $(0.008)$ & $(0.010)$ & $(0.012)$ \\
$\widehat{H}$ & - & - & - & - & 0.006 & 0.010 & 0.010 & 0.018 & 0.008 & 0.008 \\
& & & & & $(0.005)$ & $(0.008)$ & $(0.005)$ & $(0.007)$ & $(0.006)$ & $(0.010)$ \\
\hline $\mathcal{Q}(\widehat{\nu})$ & 38.546 & {$[0.000]$} & 16.828 & {$[0.000]$} & 13.079 & {$[0.000]$} & 10.038 & {$[0.002]$} & 10.154 & {$[0.001]$} \\
\hline \hline
\end{tabular}

Note: Standard errors in parenthesis, p-values in brackets. The set of instrument is composed of the average $(\mathrm{c} / \mathrm{y})$ over the sub-sample, the average growth rate of population over the 15 first periods of the sub-sample.

Table 10: Fixed Effects and Openness

\begin{tabular}{lcccccccc}
\hline \hline & \multicolumn{2}{c}{$\mathrm{OP}<\operatorname{med}(\mathrm{OP})$} & \multicolumn{2}{c}{$\mathrm{OP} \geqslant \operatorname{med}(\mathrm{OP})$} & \multicolumn{2}{c}{$\Delta \mathrm{OP}<\operatorname{med}(\Delta \mathrm{OP})$} & \multicolumn{2}{c}{$\Delta \mathrm{OP} \geqslant \operatorname{med}(\Delta \mathrm{OP})$} \\
& $60-78$ & $78-98$ & $60-78$ & $78-98$ & $60-78$ & $78-98$ & $60-78$ & $78-98$ \\
\hline$\widehat{y}_{0}$ & -0.053 & -0.057 & -0.042 & -0.045 & -0.050 & -0.057 & -0.052 & -0.057 \\
& $(0.008)$ & $(0.009)$ & $(0.007)$ & $(0.009)$ & $(0.008)$ & $(0.009)$ & $(0.005)$ & $(0.006)$ \\
$\widehat{\nu}$ & 0.034 & 0.045 & 0.025 & 0.055 & 0.044 & 0.065 & 0.017 & 0.044 \\
& $(0.009)$ & $(0.007)$ & $(0.007)$ & $(0.011)$ & $(0.010)$ & $(0.012)$ & $(0.005)$ & $(0.007)$ \\
\hline $\mathcal{Q}(\widehat{\nu})$ & 2.219 & {$[0.136]$} & 14.466 & {$[0.000]$} & 6.934 & {$[0.008]$} & 13.790 & {$[0.000]$} \\
\hline \hline
\end{tabular}

Note: Standard errors in parenthesis, p-values in brackets. The set of instrument is composed of the average (c/y) over the sub-sample, the average growth rate of population over the 15 first periods of the sub-sample. 


\section{E Proof of Propositions}

In this section we will abstract from any reference to the index of the economy, except when strictly necessary, in order to save on notation.

Proof of proposition 1: In the absence of trade $X_{1 t}=X_{2 t}=0$ so that the problem the final good producer has to solve is

$$
\max _{Z_{1 t}, Z_{2 t}} Z_{1 t}^{\varphi} Z_{2 t}^{1-\varphi}-P_{1 t} Z_{1 t}-P_{2 t} Z_{2 t}
$$

This yields the standard input demand functions $\varphi Y_{t}=P_{1 t} Z_{1 t}$ and $(1-\varphi) Y_{t}=P_{2 t} Z_{2 t}$.

Free entry on the final good market implies that $1=\frac{P_{1 t}^{\varphi} P_{2 t}^{1-\varphi}}{\Phi}$ with $\Phi=\varphi^{\varphi}(1-\varphi)^{1-\varphi}$. Then, defining the relative price $p_{t}=P_{2 t} / P_{1 t}$, we have

$$
P_{1 t}=\Phi p_{t}^{\varphi-1} \text { and } P_{2 t}=\Phi p_{t}^{\varphi}
$$

In the first intermediate good sector, the representative producer solves

$$
\max _{K_{1 t}, K_{1 t}} P_{1 t} \Theta_{1} K_{1 t}^{\alpha}\left(\Gamma_{t} L_{1 t}\right)^{1-\alpha}-W_{1 t} K_{1 t}-q_{t} K_{1 t}
$$

which leads to the standard input demand functions, $\alpha P_{1 t} Z_{1 t}=q_{t} K_{1 t}$ and $(1-\alpha) P_{1 t} Z_{1 t}=W_{1 t} L_{1 t}$. Likewise, in sector 2, we have $\beta P_{2 t} Z_{2 t}=q_{t} K_{2 t}$ and $(1-\beta) P_{2 t} Z_{2 t}=W_{2 t} L_{2 t}$. Finally, surplus maximization by the trade unions subject to labor demands leads to the wage setting rule $W_{2 t}=\frac{\theta}{\theta-\beta} W_{1 t}=(1+x) W_{1 t}$.

In equilibrium, we have $K_{1 t}+K_{2 t}=K_{t}$ and $L_{1 t}+L_{2 t}=L_{t}$ such that solving the system composed of demand functions for intermediate good, capital and labor in each sector and making use of the wage setting rule, we easily get

$$
\begin{array}{rlrl}
K_{1 t}=\frac{\alpha \varphi}{\alpha \varphi+\beta(1-\varphi)} K_{t} & K_{2 t} & =\frac{\beta(1-\varphi)}{\alpha \varphi+\beta(1-\varphi)} K_{t} \\
L_{1 t}=\frac{\varphi(1-\alpha)}{\varphi(1-\alpha)+(1-\varphi) \frac{1-\beta}{1+x}} L_{t} & L_{2 t}=\frac{(1-\varphi) \frac{1-\beta}{1+x}}{\varphi(1-\alpha)+(1-\varphi) \frac{1-\beta}{1+x}} L_{t}
\end{array}
$$

Therefore, we can compute final output as

$$
Y_{t}=\Upsilon\left(\Gamma_{t}\right) K_{t}^{\alpha \varphi+\beta(1-\varphi)} L_{t}^{1-\alpha \varphi-\beta(1-\varphi)}
$$

where

$$
\Upsilon\left(\Gamma_{t}\right)=\Theta_{1}^{\varphi} \Theta_{2}^{1-\varphi} \frac{(\alpha \varphi)^{\alpha \varphi}(\beta(1-\varphi))^{\beta(1-\varphi)}}{[\alpha \varphi+\beta(1-\varphi)]^{\alpha \varphi+\beta(1-\varphi)}} \frac{((1-\alpha) \varphi)^{(1-\alpha) \varphi}\left((1-\varphi) \frac{1-\beta}{1+x}\right)^{(1-\beta)(1-\varphi)}}{\left[(1-\alpha) \varphi+(1-\varphi) \frac{1-\beta}{1+x}\right]^{\varphi(1-\alpha)+(1-\varphi)(1-\beta)}} \Gamma_{t}^{1-\alpha \varphi-\beta(1-\varphi)}
$$

such that output-per-worker $y_{t}=Y_{t} / L_{t}$ expresses, in terms of capital-per-worker $k_{t}=K_{t} / L_{t}$, as

$$
y_{t}=\Upsilon\left(\Gamma_{t}\right) k_{t}^{\alpha \varphi+\beta(1-\varphi)}
$$

Hence the dynamics of the economy — in intensive form - may be summarized by

$$
(1+\gamma)(1+n) \frac{k_{t+1}}{\Gamma_{t+1}}=s \Upsilon\left(\frac{k_{t}}{\Gamma_{t}}\right)^{\alpha \varphi+\beta(1-\varphi)}+(1-\delta)\left(\frac{k_{t}}{\Gamma_{t}}\right)
$$


where $\Upsilon=\Upsilon\left(\Gamma_{t}\right) / \Gamma_{t}^{1-\alpha \varphi-\beta(1-\varphi)}$ which admits

$$
k^{\star}=\left(\frac{s \Upsilon}{(1+\gamma)(1+n)+\delta-1}\right)^{\frac{1}{1-\alpha \varphi-\beta(1-\varphi)}}=(\nu \Upsilon)^{\frac{1}{1-\alpha \varphi-\beta(1-\varphi)}}
$$

as steady state.

Q.E.D

Proof of proposition 2: In the autarkic economy, private $\left(r_{t}^{\mathrm{A}}\right)$ and social $\left(z_{t}^{\mathrm{A}}\right)$ returns to capital are the same. Indeed, the rental rate of capital, in terms of good 1, is given by

$$
q_{t}=\alpha P_{1 t} \frac{Z_{1 t}}{K_{1 t}}=\beta P_{2 t} \frac{Z_{2 t}}{K_{2 t}}
$$

In equilibrium, we have $P_{1 t} Z_{1 t}=\varphi Y_{t}$ and $P_{2 t} Z_{2 t}=(1-\varphi) Y_{t}$, therefore

$$
q_{t}=\alpha \varphi \frac{Y_{t}}{K_{1 t}}=\beta(1-\varphi) \frac{Y_{t}}{K_{2 t}}=(\alpha \varphi+\beta(1-\varphi)) \frac{Y_{t}}{K_{t}}
$$

Hence, the returns to capital, in terms of the final good, are given by

$$
r_{t}^{\mathrm{A}}=z_{t}^{\mathrm{A}}=q_{t}=(\alpha \varphi+\beta(1-\varphi)) \frac{Y_{t}}{K_{t}}=(\alpha \varphi+\beta(1-\varphi)) \Upsilon\left(\Gamma_{t}\right) k_{t}^{\alpha \varphi+\beta(1-\varphi)-1}
$$

Q.E.D

Proof of Proposition 3: In the closed economy, the aggregate production function in economy $i$ along a steady growth path is given by

$$
y_{i}=\Upsilon(\Gamma)^{\frac{1}{1-\alpha \varphi-\beta(1-\varphi)}} \nu_{i}^{\frac{\alpha \varphi+\beta(1-\varphi)}{1-\alpha \varphi-\beta(1-\varphi)}}
$$

where $\nu \equiv s /((1+\gamma)(1+n)-(1-\delta))$. Let us consider the $(\log -)$ difference between output per worker in economy $i$ and in the big economy, $\widehat{y}=\log \left(y_{i}\right)-\log \left(y_{0}\right)$, where 0 denotes the big economy. Let us define $\widehat{\nu}_{i}=\log \left(\nu_{i}\right)-\log \left(\nu_{0}\right)$, we then have

$$
\widehat{y}_{i}=g\left(\widehat{\nu}_{i}\right)=\frac{\alpha \varphi+\beta(1-\varphi)}{1-\alpha \varphi-\beta(1-\varphi)} \widehat{\nu}_{i}
$$

Making use of the change of variable formula, and denoting by $\mu^{\widehat{\nu}}(\cdot)$ the distribution of $\widehat{\nu}$, we have

$$
\mu^{\widehat{Y}}(\widehat{y})=\frac{1-\alpha \varphi-\beta(1-\varphi)}{\alpha \varphi+\beta(1-\varphi)} \mu^{\nu}\left(\frac{1-\alpha \varphi-\beta(1-\varphi)}{\alpha \varphi+\beta(1-\varphi)} \widehat{y}\right)
$$

Q.E.D

Proof of lemma 1: In the small open economy, each firm producing the final good takes the price of goods as given, such that final output is given by

$$
Y_{t}=P_{1 t} Z_{1 t}+P_{2 t} Z_{2 t}
$$

The intermediate goods producers problem may be rewritten as

$$
\max _{K_{1 t}, K_{2 t}, L_{1 t}, L_{2 t}, Z_{1 t}, Z_{2 t}} P_{1 t} Z_{1 t}+P_{2 t} Z_{2 t}-q_{t} K_{t}-W_{1 t} L_{1 t}-W_{2 t} L_{2 t}
$$


subject to

$$
\left\{\begin{array}{l}
Z_{1 t} \leqslant \Theta_{1} K_{1 t}^{\alpha}\left(\Gamma_{t} L_{1 t}\right)^{1-\alpha} \\
Z_{2 t} \leqslant \Theta_{2} K_{2 t}^{\beta}\left(\Gamma_{t} L_{2 t}\right)^{1-\beta} \\
K_{1 t}+K_{2 t} \leqslant K_{t} \\
L_{1 t}+L_{2 t} \leqslant L_{t} \\
L_{1 t} \geqslant 0, L_{2 t} \geqslant 0, K_{1 t} \geqslant 0, K_{2 t} \geqslant 0 \\
W_{2 t}=(1+x) W_{1 t}
\end{array}\right.
$$

Since technology is strictly increasing in inputs, the first four constraints ought to bind, such that the problem simplifies to

$\max _{K_{1 t}, K_{t}, L_{1 t}, L_{t}} P_{1 t} \Theta_{1} K_{1 t}^{\alpha}\left(\Gamma_{t} L_{1 t}\right)^{1-\alpha}+P_{2 t} \Theta_{2}\left(K_{t}-K_{1 t}\right)^{\beta}\left(\Gamma_{t}\left(L_{t}-L_{1 t}\right)\right)^{1-\beta}-q_{t} K_{t}-W_{1 t} L_{1 t}-(1+x) W_{1 t}\left(L_{t}-L_{1 t}\right)$

subject to $L_{1 t} \geqslant 0, L_{t} \geqslant L_{1 t}, K_{1 t} \geqslant 0, K_{t} \geqslant K_{1 t}$ to which we associate the lagrange multipliers $\lambda_{L}^{0}, \lambda_{L}^{1}, \lambda_{K}^{0}, \lambda_{K}^{1}$. This leads to the following set of optimality conditions

$$
\begin{aligned}
& \beta P_{2 t} \Theta_{2}\left(\frac{K_{t}-K_{1 t}}{L_{t}-L_{1 t}}\right)^{\beta-1} \Gamma_{t}^{1-\beta}=q_{t} \\
& (1-\beta) P_{2 t} \Theta_{2}\left(\frac{K_{t}-K_{1 t}}{L_{t}-L_{1 t}}\right)^{\beta} \Gamma_{t}^{1-\beta}=(1+x) W_{1 t} \\
& \alpha P_{1 t} \Theta_{1}\left(\frac{K_{1 t}}{L_{1 t}}\right)^{\alpha-1} \Gamma_{t}^{1-\alpha}-\beta P_{2 t} \Theta_{2}\left(\frac{K_{t}-K_{1 t}}{L_{t}-L_{1 t}}\right)^{\beta-1} \Gamma_{t}^{1-\beta}=\lambda_{K t}^{1}-\lambda_{K t}^{0} \\
& (1-\alpha) P_{1 t} \Theta_{1}\left(\frac{K_{1 t}}{L_{1 t}}\right)^{\alpha} \Gamma_{t}^{1-\alpha}-(1-\beta) P_{2 t} \Theta_{2}\left(\frac{K_{t}-K_{1 t}}{L_{t}-L_{1 t}}\right)^{\beta} \Gamma_{t}^{1-\beta}-x W_{1 t}=\lambda_{L t}^{1}-\lambda_{L t}^{0}
\end{aligned}
$$

An interior solution, for which $K_{1 t}, K_{2 t}, L_{1 t}, L_{2 t}>0$ - which corresponds to a specialization phase implies that $K_{1 t}, K_{2 t}, L_{1 t}$ and $L_{2 t}$ satisfy (using (22)-(24))

$$
\frac{K_{2 t}}{L_{2 t}}=\frac{K_{t}-K_{1 t}}{L_{t}-L_{1 t}}=\frac{\beta(1-\alpha)(1+x)}{\alpha(1-\beta)} \frac{K_{1 t}}{L_{1 t}}
$$

Let us first study the conditions under which an economy chooses to specialize in the production of type 1 intermediate good. In this case, $K_{1 t}=K_{t}$ and $L_{1 t}=L_{t}$, which implies that $\lambda_{K t}^{0}=\lambda_{L t}^{0}=0$ and $\lambda_{K t}^{1} \geqslant 0$ and $\lambda_{L t}^{1} \geqslant 0$. Therefore, equations (22)-(24), evaluated along (25), satisfy

$$
\begin{aligned}
& \alpha P_{1 t} \Theta_{1}\left(\frac{K_{1 t}}{L_{1 t}}\right)^{\alpha-1} \Gamma_{t}^{1-\alpha}-\beta P_{2 t} \Theta_{2}\left(\frac{\beta(1-\alpha)(1+x)}{\alpha(1-\beta)} \frac{K_{1 t}}{L_{1 t}}\right)^{\beta-1} \Gamma_{t}^{1-\beta} \geqslant 0 \\
& (1-\alpha) P_{1 t} \Theta_{1}\left(\frac{K_{1 t}}{L_{1 t}}\right)^{\alpha} \Gamma_{t}^{1-\alpha}-\frac{1-\beta}{1+x} P_{2 t} \Theta_{2}\left(\frac{\beta(1+x)(1-\alpha)}{\alpha(1-\beta)} \frac{K_{1 t}}{L_{1 t}}\right)^{\beta-1} \Gamma_{t}^{1-\beta} \geqslant 0
\end{aligned}
$$

which triggers that

$$
\frac{K_{1 t}}{L_{1 t}}=\frac{K_{t}}{L_{t}} \leqslant \underline{k}\left(p_{t}, \Gamma_{t}\right) \equiv \Gamma_{t}\left(p_{t} \frac{\Theta_{2}}{\Theta_{1}}\right)^{\frac{1}{\alpha-\beta}}\left(\frac{\beta}{\alpha}\right)^{\frac{\beta}{\alpha-\beta}}\left(\frac{1-\beta}{(1-\alpha)(1+x)}\right)^{\frac{1-\beta}{\alpha-\beta}}
$$

where $p_{t}=P_{2 t} / P_{1 t}$.

Let us now study the conditions under which an economy chooses to specialize in the production of type 2 intermediate good. In this case, $K_{2 t}=K_{t}$ and $L_{2 t}=L_{t}$, which implies that $\lambda_{K t}^{1}=\lambda_{L t}^{1}=0$ and $\lambda_{K t}^{0} \geqslant 0$ and $\lambda_{L t}^{0} \geqslant 0$. Therefore, equations (22)-(24), evaluated along (25), satisfy

$$
\begin{aligned}
& \alpha P_{1 t} \Theta_{1}\left(\frac{\alpha(1-\beta)}{\beta(1-\alpha)(1+x)} \frac{K_{t}-K_{1 t}}{L_{t}-L_{1 t}}\right)^{\alpha-1} \Gamma_{t}^{1-\alpha}-\beta P_{2 t} \Theta_{2}\left(\frac{K_{t}-K_{1 t}}{L_{t}-L_{1 t}}\right)^{\beta-1} \Gamma_{t}^{1-\beta} \leqslant 0 \\
& (1-\alpha) P_{1 t} \Theta_{1}\left(\frac{\alpha(1-\beta)}{\beta(1-\alpha)(1+x)} \frac{K_{t}-K_{1 t}}{L_{t}-L_{1 t}}\right)^{\alpha} \Gamma_{t}^{1-\alpha}-\frac{1-\beta}{1+x} P_{2 t} \Theta_{2}\left(\frac{K_{t}-K_{1 t}}{L_{t}-L_{1 t}}\right)^{\beta-1} \Gamma_{t}^{1-\beta} \leqslant 0
\end{aligned}
$$


which triggers that

$$
\frac{K_{t}-K_{1 t}}{L_{t}-L_{1 t}}=\frac{K_{t}}{L_{t}} \geqslant \bar{k}\left(p_{t}, \Gamma_{t}\right) \equiv \Gamma_{t}\left(p_{t} \frac{\Theta_{2}}{\Theta_{1}}\right)^{\frac{1}{\alpha-\beta}}\left(\frac{\beta}{\alpha}\right)^{\frac{\alpha}{\alpha-\beta}}\left(\frac{1-\beta}{(1-\alpha)(1+x)}\right)^{\frac{1-\alpha}{\alpha-\beta}}
$$

Q.E.D

Proof of proposition 4: We have to study three cases, depending on the level of the capital per efficient unit of labor.

- $k_{t} \leqslant \underline{k}\left(p_{t}, \Gamma_{t}\right):$ In this case, the economy fully specializes in the production of type 1 intermediate good, we therefore have $y_{t}=P_{1 t} z_{1 t}=P_{1 t} \Theta_{1} k_{t}^{\alpha} \Gamma_{t}^{1-\alpha}$, where $y_{t}=Y_{t} / L_{t}$ and $z_{1 t}=Z_{1 t} / L_{t}$. Since $P_{1 t}$ is given by (18), we finally have $y_{t}=\Phi p_{t}^{\varphi-1} \Theta_{1} k_{t}^{\alpha} \Gamma_{t}^{1-\alpha}$.

- $k_{t} \geqslant \bar{k}\left(p_{t}, \Gamma_{t}\right):$ In this case, the economy fully specializes in the production of type 2 intermediate good, we therefore have $y_{t}=P_{2 t} z_{2 t}=P_{2 t} \Theta_{2} k_{t}^{\beta} \Gamma_{t}^{1-\beta}$, where $z_{2 t}=Z_{2 t} / L_{t}$. Since $P_{2 t}$ is given by (18), we finally have $y_{t}=\Phi p_{t}^{\varphi} \Theta_{2} k_{t}^{\beta} \Gamma_{t}^{1-\beta}$.

- $\underline{\underline{k}}\left(p_{t}, \Gamma_{t}\right) \leqslant k \leqslant \bar{k}\left(p_{t}, \Gamma_{t}\right)$ : In this case, the economy lies in the specialization process, and we have

$$
y_{t}=P_{1 t} z_{1 t}+P_{2 t} z_{2 t}
$$

We therefore have to solve the allocation of capital and labor problem. This implies solving the set of equations

$$
\begin{aligned}
& \alpha P_{1 t} \Theta_{1}\left(\frac{K_{1 t}}{L_{1 t}}\right)^{\alpha-1} \Gamma_{t}^{1-\alpha}=\beta P_{2 t} \Theta_{2}\left(\frac{K_{t}-K_{1 t}}{L_{t}-L_{1 t}}\right)^{\beta-1} \Gamma_{t}^{1-\beta} \\
& (1-\alpha) P_{1 t} \Theta_{1}\left(\frac{K_{1 t}}{L_{1 t}}\right)^{\alpha} \Gamma_{t}^{1-\alpha}=\frac{1-\beta}{1+x} P_{2 t} \Theta_{2}\left(\frac{K_{t}-K_{1 t}}{L_{t}-L_{1 t}}\right)^{\beta} \Gamma_{t}^{1-\beta}
\end{aligned}
$$

which implies that

$$
\begin{aligned}
& \frac{K_{t}-K_{1 t}}{L_{t}-L_{1 t}}=\frac{\beta(1-\alpha)(1+x)}{\alpha(1-\beta)} \frac{K_{1 t}}{L_{1 t}} \\
& \frac{K_{1 t}}{L_{1 t}}=\Gamma_{t}\left(p_{t} \frac{\Theta_{2}}{\Theta_{1}}\right)^{\frac{1}{\alpha-\beta}}\left(\frac{\beta}{\alpha}\right)^{\frac{\beta}{\alpha-\beta}}\left(\frac{1-\beta}{(1-\alpha)(1+x)}\right)^{\frac{1-\beta}{\alpha-\beta}}=\underline{k}\left(p_{t}, \Gamma_{t}\right)
\end{aligned}
$$

Let us then denote $\sigma_{L t}=L_{1 t} / L_{t}$ and $\sigma_{K t}=K_{1 t} / K_{t}$. Solving (28) and (29), we get

$$
\begin{aligned}
\sigma_{L t} & =\frac{\beta(1-\alpha)}{\beta(1-\alpha)-\alpha \frac{1-\beta}{1+x}}-\frac{\alpha \frac{1-\beta}{1+x}}{\beta(1-\alpha)-\alpha \frac{1-\beta}{1+x}} \frac{k_{t}}{\underline{k}\left(p_{t}, \Gamma_{t}\right)} \\
\sigma_{K t} & =\sigma_{L t} \frac{\underline{k}\left(p_{t}, \Gamma_{t}\right)}{k_{t}}
\end{aligned}
$$

We therefore easily get

$$
\begin{aligned}
P_{1 t} z_{1 t} & =P_{1 t} \Theta_{1} \underline{k}\left(p_{t}\right)^{\alpha} \Gamma_{t}^{1-\alpha} \sigma_{L t} \\
& =\frac{\beta(1-\alpha)}{\beta(1-\alpha)-\alpha \frac{1-\beta}{1+x}} P_{1 t} \Theta_{1} \underline{k}\left(p_{t}, \Gamma_{t}\right)^{\alpha} \Gamma_{t}^{1-\alpha}-\frac{\alpha \frac{1-\beta}{1+x}}{\beta(1-\alpha)-\alpha \frac{1-\beta}{1+x}} P_{1 t} \Theta_{1} \Gamma_{t}^{1-\alpha} \underline{k}\left(p_{t}, \Gamma_{t}\right)^{\alpha-1} k_{t}
\end{aligned}
$$

Likewise, straightforward calculation gives

$$
\begin{aligned}
1-\sigma_{L t} & =\frac{\alpha \frac{1-\beta}{1+x}}{\beta(1-\alpha)-\alpha \frac{1-\beta}{1+x}}\left(\frac{k_{t}}{\underline{k}\left(p_{t}, \Gamma_{t}\right)}-1\right) \\
1-\sigma_{K t} & =\frac{\beta(1-\alpha)(1+x)}{\alpha(1-\beta)} \frac{\underline{k}\left(p_{t}, \Gamma_{t}\right)}{k_{t}}\left(1-\sigma_{L t}\right)
\end{aligned}
$$


We therefore easily get

$$
\begin{aligned}
P_{2 t} z_{2 t} & =P_{2 t} \Theta_{2}\left(\frac{\beta(1-\alpha)(1+x)}{\alpha(1-\beta)} \underline{k}\left(p_{t}, \Gamma_{t}\right)\right)^{\beta} \Gamma_{t}^{1-\beta}\left(1-\sigma_{L t}\right) \\
& =P_{2 t} \Theta_{2}\left(\frac{\beta(1-\alpha)(1+x)}{\alpha(1-\beta)} \underline{k}\left(p_{t}, \Gamma_{t}\right)\right)^{\beta} \Gamma_{t}^{1-\beta} \frac{\alpha \frac{1-\beta}{1+x}}{\beta(1-\alpha)-\alpha \frac{1-\beta}{1+x}}\left(\frac{k_{t}}{\underline{k}\left(p_{t}, \Gamma_{t}\right)}-1\right)
\end{aligned}
$$

Then, after simple although tedious algebra and making use of (18), we get

$$
y_{t}=B\left(p_{t}\right) \Gamma_{t}+A\left(p_{t}\right) k_{t}
$$

where

$$
\begin{aligned}
& B\left(p_{t}\right)=\Phi p_{t}^{\varphi-1} \frac{(\beta-\alpha)(1-\alpha)}{\beta(1-\alpha)-\alpha \frac{1-\beta}{1+x}}\left(p_{t} \Theta_{2}\right)^{\frac{\alpha}{\alpha-\beta}} \Theta_{1}^{\frac{\beta}{\beta-\alpha}}\left(\frac{\beta}{\alpha}\right)^{\frac{\alpha \beta}{\alpha-\beta}}\left(\frac{1-\beta}{(1-\alpha)(1+x)}\right)^{\frac{\alpha(1-\beta)}{\alpha-\beta}} \\
& A\left(p_{t}\right)=\Phi p_{t}^{\varphi-1} \frac{\alpha\left(1-\alpha-\frac{1-\beta}{1+x}\right)}{\beta(1-\alpha)-\alpha \frac{1-\beta}{1+x}}\left(p_{t} \Theta_{2}\right)^{\frac{1-\alpha}{\beta-\alpha}} \Theta_{1}^{\frac{1-\beta}{\alpha-\beta}}\left(\frac{\beta}{\alpha}\right)^{\frac{\beta(1-\alpha)}{\beta-\alpha}}\left(\frac{1-\beta}{(1-\alpha)(1+x)}\right)^{\frac{(1-\beta)(1-\alpha)}{\beta-\alpha}}
\end{aligned}
$$

Q.E.D

Proof of proposition 5: Under free trade, the private returns to capital, $\left(r_{t}^{\mathrm{FT}}\right)$, are given by (see proof of lemma 1, equation (21))

$$
r_{t}^{\mathrm{FT}}=q_{t}=\beta P_{2 t} \Theta_{2}\left(\frac{K_{t}-K_{1 t}}{L_{t}-L_{1 t}}\right)^{\beta-1} \Gamma_{t}^{1-\beta}
$$

which rewrites

$$
r_{t}^{\mathrm{FT}}=\beta P_{2 t} \Theta_{2} k_{t}^{\beta-1}\left(\frac{1-\sigma_{K t}}{1-\sigma_{L t}}\right)^{\beta-1} \Gamma_{t}^{1-\beta}=\beta P_{2 t} \Theta_{2}\left(\frac{\beta(1-\alpha)(1+x)}{\alpha(1-\beta)} \underline{k}\left(p_{t}, \Gamma_{t}\right)\right)^{\beta-1} \Gamma_{t}^{1-\beta}
$$

Plugging the definition of $\underline{k}\left(p_{t}, \Gamma_{t}\right)$ and that of $P_{2 t}$ in the latter equation, we get

$$
r_{t}^{\mathrm{FT}}=\beta \Phi p_{t}^{\varphi-1}\left(p_{t} \Theta_{2}\right)^{\frac{1-\alpha}{\beta-\alpha}} \Theta_{1}^{\frac{1-\beta}{\alpha-\beta}}\left(\frac{\beta}{\alpha}\right)^{-\frac{\alpha(1-\beta)}{\alpha-\beta}}\left(\frac{1-\beta}{(1-\alpha)(1+x)}\right)^{-\frac{(1-\alpha)(1-\beta)}{\alpha-\beta}}
$$

Further from the optimal allocation of $Z_{1}$ and $Z_{2}$ in the big economy (autarkic world), we have

$$
p_{t}=\frac{1-\varphi}{\varphi} \frac{Z_{1 t}}{Z_{2 t}}
$$

Using the value of $z_{1 t}$ and $z_{2 t}$, the relative price, $p_{t}$, evaluated at the steady growth path of the big economy (indexed by 0 ) is given by

$$
p^{\star}=\frac{\Theta_{1}}{\Theta_{2}} \frac{\alpha^{\alpha}(\theta(1-\alpha))^{1-\alpha}}{\beta^{\beta}\left(\frac{1-\beta}{1+x}\right)^{1-\beta}}\left(\frac{\alpha \varphi+\beta(1-\varphi)}{(1-\alpha) \varphi+(1-\varphi) \frac{1-\beta}{1+x}}\right)^{\beta-\alpha}\left(\frac{k_{0 t}}{\Gamma_{t}}\right)^{\alpha-\beta}
$$

Plugging this expression in the definition of $\underline{k}(p, \Gamma)$, we can express the private return to capital (at the steady state of the big economy) as

$$
\begin{aligned}
r_{t}^{\mathrm{FT}}= & \Theta_{1}^{\varphi} \Theta_{2}^{1-\varphi} \frac{(\alpha \varphi)^{\alpha \varphi}(\varphi(1-\alpha))^{\varphi(1-\alpha)}(\beta(1-\varphi))^{\beta(1-\varphi)}\left((1-\varphi) \frac{1-\beta}{1+x}\right)^{(1-\beta)(1-\varphi)}}{(\alpha \varphi+\beta(1-\varphi))^{\alpha \varphi+\beta(1-\varphi)}\left(\varphi(1-\alpha)+(1-\varphi) \frac{1-\beta}{1+x}\right)^{1-\alpha \varphi-\beta(1-\varphi)}} \cdots \\
& \times(\alpha \varphi+\beta(1-\varphi)) k_{0 t}^{\alpha \varphi+\beta(1-\varphi)-1} \Gamma_{t}^{1-\alpha \varphi-\beta(1-\varphi)}
\end{aligned}
$$


or

$$
r_{t}^{\mathrm{FT}}=(\alpha \varphi+\beta(1-\varphi)) \Upsilon\left(\Gamma_{t}\right) k_{0 t}^{\alpha \varphi+\beta(1-\varphi)-1}=r_{t}^{\mathrm{A}}
$$

We now consider the social return to capital, which is now obtained by deriving the aggregate production function when the economy produces both goods. Hence, we have $z_{t}^{\mathrm{FT}}=A\left(p_{t}\right)$. Using the definition of $A$ (see proposition 4 ) and the expression for $p^{\star}$, the social return to capital in the steady state of the big economy is given by

$$
z_{t}^{\mathrm{FT}}=\frac{1-\alpha-\frac{1-\beta}{1+x}}{\beta(1-\alpha)-\alpha \frac{1-\beta}{1+x}}(\alpha \varphi+\beta(1-\varphi)) B\left(\Gamma_{t}\right) k_{t}^{\star \alpha \varphi+\beta(1-\varphi)-1}=\frac{1-\alpha-\frac{1-\beta}{1+x}}{\beta(1-\alpha)-\alpha \frac{1-\beta}{1+x}} z^{\mathrm{A}}
$$

It is then straightforward to verify that as long as $\alpha, \beta \in(0,1)$ and $x>0$ the multiplier term is greater than 1 , such that $z^{\mathrm{FT}} \geqslant z^{\mathrm{A}}$.

Q.E.D

Proof of Proposition 6: Given the form of the production function reported in Figure 3, the model admits 1,3 or an infinity of non trivial steady state (the trivial steady state being 0 ).

- Let us first consider the case where we have an infinity of steady state. This occurs when the ray $k / \nu$ overlies the linear part of the production function, which can only happen if and only if that the linear part reduced to $A(p) k$. This situation would then corresponds to $B(p)=0$ or identically to a real wage rate equal to zero. But then the demand for labor would be infinite while the supply is finite, therefore driving the real wage up. This is clearly not an equilibrium.

- A second possibility of multiple equilibria is to have 3 steady states. But the form of the function triggers that a first steady state lies in the area where the economy is fully specialized in the production of type 1 intermediate goods, one in the zone of full specialization in type 2 intermediate goods, and one in the specialization area. This can only be the case if the linear part of the production function crosses the ray $k / \nu$ from below, which triggers a negative $B(p)$ and therefore negative wages. Hence, as explained above, this cannot be an equilibrium.

Therefore, there exists a unique equilibrium.

Q.E.D

Proof of lemma 2: We first start by characterizing the two boundary values $\underline{\widehat{\nu}}$ and $\bar{\nu}$.

- Let us compute $\underline{\nu}\left(p_{t}, \Gamma_{t}\right)=\underline{k}\left(p_{t}, \Gamma_{t}\right) / \underline{y}\left(p_{t}, \Gamma_{t}\right)$. At this particular value, we have $y_{i t}=P_{1 t} z_{1 i t}$, such that

$$
\underline{\nu}\left(p_{t}, \Gamma_{t}\right)=\frac{p_{t}^{1-\varphi}}{\Phi} \frac{\underline{k}\left(p_{t}, \Gamma_{t}\right)^{1-\alpha}}{\Theta_{1} \Gamma_{t}^{1-\alpha}}
$$

Plugging into the previous equation the definition of $\underline{k}\left(p_{t}, \Gamma_{t}\right)$ and the definition of $p_{t}$ along the steady growth path of the big economy (Equation (35)), we have

$$
\underline{\nu}\left(p_{t}, \Gamma_{t}\right)=\frac{\alpha}{\alpha \varphi+\beta(1-\varphi)} \frac{k_{0 t}}{\Upsilon\left(\Gamma_{t}\right) k_{0 t}^{\alpha \varphi+\beta(1-\varphi)}}=\frac{\alpha}{\alpha \varphi+\beta(1-\varphi)} \frac{k_{0 t}}{y_{0 t}}=\frac{\alpha}{\alpha \varphi+\beta(1-\varphi)} \nu_{0}
$$

Hence, we have $\underline{\widehat{\nu}} \equiv \log \left(\underline{\nu}\left(p_{t}, \gamma_{t}\right)\right)-\log \left(\nu_{0}\right)=\log \left(\frac{\alpha}{\alpha \varphi+\beta(1-\varphi)}\right)$

- Let us now compute $\bar{\nu}\left(p_{t}, \Gamma_{t}\right)=\bar{k}\left(p_{t}, \Gamma_{t}\right) / \bar{y}\left(p_{t}, \Gamma_{t}\right)$. At this particular value, we have $y_{i t}=P_{2 t} z_{2 i t}$, such that

$$
\underline{\nu}\left(p_{t}, \Gamma_{t}\right)=\frac{p_{t}^{-\varphi}}{\Phi} \frac{\underline{k}\left(p_{t}, \Gamma_{t}\right)^{1-\beta}}{\Theta_{2} \Gamma_{t}^{1-\beta}}
$$


Plugging into the previous equation the definition of $\bar{k}\left(p_{t}, \Gamma_{t}\right)$ and the definition of $p$ at the steady state of the big economy, we have

$$
\bar{\nu}\left(p_{t}, \Gamma_{t}\right)=\frac{\beta}{\alpha \varphi+\beta(1-\varphi)} \frac{k_{0, t}}{\Upsilon\left(\Gamma_{t}\right) k_{0, t}{ }^{\alpha \varphi+\beta(1-\varphi)}}=\frac{\beta}{\alpha \varphi+\beta(1-\varphi)} \frac{k_{0, t}}{y_{0, t}}=\frac{\beta}{\alpha \varphi+\beta(1-\varphi)} \nu_{0}
$$

Hence, we have $\bar{\nu} \equiv \log \left(\bar{\nu}\left(p_{t}, \Gamma_{t}\right)\right)-\log \left(\nu_{0}\right)=\log \left(\frac{\beta}{\alpha \varphi+\beta(1-\varphi)}\right)$

We then have to consider 3 cases:

$\nu_{i}<\underline{\nu}$ : In this case $y=P_{1 t} \Theta_{1} k^{\alpha} \Gamma_{t}^{1-\alpha}$. Evaluating this along a steady growth path, we have $y_{i t}=$ $\left(\Phi p_{t}^{\varphi-1} \Theta_{1}\right)^{\frac{1}{1-\alpha}} \nu_{i}^{\frac{\alpha}{1-\alpha}} \Gamma_{t}$. Plugging the expression of the relative price along the steady growth path of the big economy (Equation (35)) in the output-per-worker in economy $i$, and remembering $i$ ) the definition of $\Upsilon\left(\Gamma_{t}\right)$, ii) the definition of output-per-worker in the big economy, we have

$$
y_{i t}=\left(\frac{\alpha \varphi+\beta(1-\varphi)}{\alpha}\right)^{\frac{\alpha}{1-\alpha}} \frac{(1-\alpha)+(1-\varphi) \frac{1-\beta}{1+x}}{1-\alpha}\left(\frac{\nu_{i}}{\nu_{0}}\right)^{\frac{\alpha}{1-\alpha}} y_{0 t}
$$

taking $\log$ on both sides, we have

$$
\widehat{y}_{i t}=\frac{\alpha}{1-\alpha} \log \left(\frac{\alpha \varphi+\beta(1-\varphi)}{\alpha}\right)+\log \left(\frac{(1-\alpha) \varphi \theta+(1-\beta)(1-\varphi)(\theta-\beta)}{\theta(1-\alpha)}\right)+\frac{\alpha}{1-\alpha} \widehat{\nu}_{i}
$$

$\underline{\nu} \leqslant \nu_{i} \leqslant \bar{\nu}: \quad$ In this case $y_{i t}=A\left(p_{t}\right) k_{i t}+B\left(p_{t}\right) \Gamma_{t}$. Evaluating this along a steady growth path, where $k_{i t}=\nu_{i} y_{i t}$, we have $y_{t}=\frac{B\left(p_{t}\right) \Gamma_{t}}{1-A\left(p_{t}\right) \nu_{i}}$. Around the steady growth path of the big economy, we have

$$
A\left(p_{t}\right)=\frac{(\alpha \varphi+\beta(1-\varphi))\left(1-\alpha-\frac{1-\beta}{1+x}\right)}{\beta(1-\alpha)-\alpha \frac{1-\beta}{1+x}} \frac{y_{0 t}}{k_{0 t}} \text { and } B\left(p_{t}\right) \Gamma_{t}=\frac{(\beta-\alpha)\left(\varphi(1-\alpha)+(1-\varphi) \frac{1-\beta}{1+x}\right)}{\beta(1-\alpha)-\alpha \frac{1-\beta}{1+x}} y_{0 t}
$$

Remembering that $\nu_{0}=\frac{k_{0 t}}{y_{0 t}}$, we then get $y_{i t}=\frac{\rho y_{0 t}}{1-(1-\rho) \frac{\nu}{\nu_{0}}}$ where $\rho=\frac{(\beta-\alpha)\left(\varphi(1-\alpha)+(1-\varphi) \frac{1-\beta}{1+x}\right)}{\beta(1-\alpha)-\alpha \frac{1-\beta}{1+x}}$ such that $\widehat{y}=\log (\rho)-\log \left(1-(1-\rho) \exp \left(\widehat{\nu}_{i}\right)\right)$.

$\nu_{i}>\bar{\nu}$ : In this case $y_{t}=P_{2 t} \Theta_{2} k_{t}^{\beta} \Gamma_{t}^{1-\beta}$. Evaluating this along a steady growth path, we have $y_{i t}=$ $\left(\Phi p_{t}^{\varphi} \Theta_{2}\right)^{\frac{1}{1-\beta}} \nu_{i}^{\frac{\beta}{1-\beta}} \Gamma_{t}$. Then, using previous results for $\nu_{i}<\underline{\nu}$, we then have, around the steady growth path of the big economy,

$$
y_{i t}=\left(\frac{\alpha \varphi+\beta(1-\varphi)}{\beta}\right)^{\frac{\beta}{1-\beta}} \frac{\varphi(1-\alpha)+(1-\varphi) \frac{1-\beta}{1+x}}{\frac{1-\beta}{1+x}}\left(\frac{\nu_{i}}{\nu_{0}}\right)^{\frac{\beta}{1-\beta}} y_{0 t}
$$

taking log on both sides, we have

$$
\widehat{y}_{i}=\frac{\beta}{1-\beta} \log \left(\frac{\alpha \varphi+\beta(1-\varphi)}{\beta}\right)+\log \left(\frac{\varphi(1-\alpha)+(1-\varphi) \frac{1-\beta}{1+x}}{\frac{1-\beta}{1+x}}\right)+\frac{\beta}{1-\beta} \widehat{\nu}_{i}
$$

Q.E.D

Proof of proposition 7: We start by computing the threshold values, $y(p)$ and $\bar{y}(p)$, for the distribution. These values are simply obtained by plugging the values for $\underline{k}(p)$ and $\bar{k}(p)$ in the relevant production functions. We therefore have

$$
\underline{y}\left(p_{t}, \Gamma_{t}\right)=P_{1 t} \Theta_{1} \underline{k}\left(p_{t}, \Gamma_{t}\right)^{\alpha} \Gamma_{t}^{1-\alpha} \text { and } \bar{y}\left(p_{t}, \Gamma_{t}\right)=P_{2 t} \Theta_{2} \bar{k}\left(p_{t}, \Gamma_{t}\right)^{\beta} \Gamma_{t}^{1-\beta}
$$


using the definition of $\underline{k}\left(p_{t}, \Gamma_{t}\right), \bar{k}\left(p_{t}, \Gamma_{t}\right), P_{1 t}$ and $P_{2 t}$, we get

$$
\underline{y}\left(p_{t}, \Gamma_{t}\right)=\Phi \Gamma_{t} p_{t}^{\varphi-1+\frac{\alpha}{\alpha-\beta}} \Theta_{1}^{\frac{\beta}{\beta-\alpha}} \Theta_{2}^{\frac{\alpha}{\alpha-\beta}}\left(\frac{\beta}{\alpha}\right)^{\frac{\alpha \beta}{\alpha-\beta}}\left(\frac{1-\beta}{(1-\alpha)(1+x)}\right)^{\frac{\alpha(1-\beta)}{\alpha-\beta}}
$$

and

$$
\bar{y}\left(p_{t}, \Gamma_{t}\right)=\Phi \Gamma_{t} p_{t}^{\varphi-1+\frac{\beta}{\alpha-\beta}} \Theta_{1}^{\frac{\beta}{\beta-\alpha}} \Theta_{2}^{\frac{\alpha}{\alpha-\beta}}\left(\frac{\beta}{\alpha}\right)^{\frac{\alpha \beta}{\alpha-\beta}}\left(\frac{1-\beta}{(1-\alpha)(1+x)}\right)^{\frac{\beta(1-\alpha)}{\alpha-\beta}}
$$

We now determine the shape of the distribution along a steady growth path, that is when $k_{i t}=\nu_{i} y_{i t}$. In this case, the relative price is given by expression (35). Hence, denoting by $y_{0 t}$ the output level along a steady growth path in the big economy, we can reexpress the thresholds as

$$
\underline{y}\left(p_{t}, \Gamma_{t}\right)=\frac{\varphi(1-\alpha)+(1-\varphi) \frac{1-\beta}{1+x}}{1-\alpha} y_{0, t} \text { and } \bar{y}\left(p_{t}, \Gamma_{t}\right)=\frac{\varphi(1-\alpha)+(1-\varphi) \frac{1-\beta}{1+x}}{\frac{1-\beta}{1+x}} y_{0, t}
$$

We now study the distribution of $\widehat{y}=\log \left(y_{t}\right)-\log \left(y_{0, t}\right)$. The direct application of the change of variable formula on the relationship reported in lemma 2 yields

$$
\mu^{\widehat{Y}}(\widehat{y})= \begin{cases}\frac{1-\alpha}{\alpha} \mu_{\widehat{\nu}}\left(\frac{1-\alpha}{\alpha}(\widehat{y}-\widehat{y})-\log \left(\frac{\alpha}{\alpha \varphi+\beta(1-\varphi)}\right)\right) & \text { if } \widehat{y}<\underline{y} \\ \frac{(1-\rho) \exp (\widehat{y})}{1-(1-\rho) \exp (\widehat{y})} \mu_{\widehat{\nu}}\left(\log \left(\frac{1-(1-\rho) \exp (\widehat{y})}{\rho}\right)\right) & \text { if } \underline{\hat{y}} \leqslant \widehat{y} \leqslant \widehat{\hat{y}} \\ \frac{1-\beta}{\beta} \mu_{\widehat{\nu}}\left(\frac{1-\beta}{\beta}(\widehat{y}-\overline{\widehat{y}})-\log \left(\frac{\beta}{\alpha \varphi+\beta(1-\varphi)}\right)\right) & \text { if } \widehat{y}>\overline{\widehat{y}}\end{cases}
$$

where $\mu_{\widehat{\nu}}(\cdot)$ is the distribution of $\widehat{\nu}=\log (\nu)-\log \left(\nu_{0}\right), \rho \equiv \frac{(\alpha \varphi+\beta(1-\varphi))\left(1-\alpha-\frac{1-\beta}{1+x}\right)}{\beta(1-\alpha)-\alpha \frac{1-\beta}{1+x}}$,

$\underline{\underline{y}} \equiv \log \left(\frac{\underline{y}(p)}{y_{0}}\right)=\log \left(\frac{\varphi(1-\alpha)+(1-\varphi) \frac{1-\beta}{1+x}}{1-\alpha}\right)$ and $\bar{y} \equiv \log \left(\frac{\bar{y}(p)}{y_{b}}\right)=\log \left(\frac{\varphi(1-\alpha)+(1-\varphi) \frac{1-\beta}{1+x}}{\frac{1-\beta}{1+x}}\right)$

Q.E.D

Proof of proposition 8 Let us first consider the autarkic case. In this situation, the aggregate production function in economy $i$ in the steady state is given by $y_{i t}=\Upsilon\left(\Gamma_{t}\right)^{\frac{1}{1-\alpha \varphi-\beta(1-\varphi)}} \nu_{i}^{\frac{\alpha \varphi-\beta(1-\varphi)}{1-\alpha(1-\varphi)}}$. Let us consider the $(\log -)$ difference between output per worker in economy $i$ and in the big economy, $\widehat{y}_{i}=\log (y)_{i}-\log \left(y_{0}\right)$. Let us define $\widehat{\nu}_{i}=\log \left(\nu_{i}\right)-\log \left(\nu_{0}\right)$, we then have $\widehat{y}_{i}=\frac{\alpha \varphi+\beta(1-\varphi)}{1-\alpha \varphi-\beta(1-\varphi)} \widehat{\nu}_{i}$, which is independent from the distorsion induced by the existence of trade union.

Let us now consider the case of an open economy, and use the relationship established in lemma 2 . We compute the sensitivity of the dispersion in the level of output to the dispersion in the long rune propensity to accumulate capital. Three cases should be considered

$\widehat{\nu}_{i}<\underline{\widehat{\nu}}$ : The sensitivity is given by $\frac{\partial \widehat{y}_{i}}{\partial \widehat{\nu}_{i}}=\frac{\alpha}{1-\alpha}$ is unaffected by the trade union markup.

$\widehat{\nu}_{i}>\overline{\widehat{\nu}}$ : The sensitivity is given by $\frac{\partial \widehat{y}_{i}}{\partial \widehat{\nu}_{i}}=\frac{\beta}{1-\beta}$ is unaffected by the trade union markup.

$\underline{\widehat{\nu}}<\widehat{\nu}_{i}<\overline{\widehat{\nu}}$ : The sensitivity is given by $\frac{\partial \widehat{y}_{i}}{\partial \widehat{\nu}_{i}}=\frac{\rho \exp \left(\widehat{\nu}_{i}\right)}{1-\rho \exp \left(\widehat{\nu}_{i}\right)}$ where $\rho \equiv \frac{(\alpha \varphi+\beta(1-\varphi))\left(1-\alpha-\frac{1-\beta}{1+x}\right)}{\beta(1-\alpha)-\alpha \frac{1-\beta}{1+x}}$. Note that $\frac{\partial^{2} \widehat{y}}{\partial \widehat{\nu} \partial \rho}=$ $\frac{\exp (\widehat{\nu})}{(1-\rho \exp (\widehat{\nu}))^{2}}>0$ such that the sensitivity of $\widehat{y}$ to $\widehat{\nu}$ is an increasing function of $\rho$. Then, note that

$$
\frac{\partial \rho}{\partial x}=\frac{(1-\alpha)(1-\beta)(\alpha \varphi+\beta(1-\varphi))}{(\beta-\alpha)(1+x)^{2}\left(\beta(1-\alpha)-\alpha \frac{1-\beta}{1+x}\right)^{2}}>0
$$


Hence, in an open economy, the larger the trade union distorsion, the greater the sensitivity of $\widehat{y}_{i}$ to $\widehat{\nu}_{i}$.

Q.E.D

Proof of Corollary 1: Let us recall that within a close economy, the dispersion of log output per worker is determined by $\widehat{y}_{i}=\frac{\alpha \varphi+\beta(1-\varphi)}{1-\alpha \varphi+\beta(1-\varphi)} \widehat{\nu}_{i}$ while, when we open trade, it changes to $\widehat{y}=\log (\rho)-$ $\log \left(1-(1-\rho) \exp \left(\widehat{\nu}_{i}\right)\right)$ which can be approximated, around the steady state of the big economy as $\widehat{y}_{i} \simeq \frac{\rho}{1-\rho} \widehat{\nu}_{i}$ where $\rho \equiv \frac{(\alpha \varphi+\beta(1-\varphi))\left(1-\alpha-\frac{1-\beta}{1+x}\right)}{\beta(1-\alpha)-\alpha \frac{1-\beta}{1+x}}$. Note that absent of trade union $(\mathrm{x}=0), \rho=\alpha \varphi+\beta(1-\varphi)$, such that we regain the dispersion in the close economy. Conversely, we saw in the proof of Proposition 8 that $\partial \rho / \partial x>0$, such that $\left.\frac{\partial \widehat{y}}{\partial \widehat{\nu}}\right|_{x>0}>\left.\frac{\partial \widehat{y}}{\partial \widehat{\nu}}\right|_{x=0}$

Q.E.D

Proof of lemma 3: Since $Y_{1}$ is unimodal and is a linear transformation of $Z$, it has to be the case that $Z$ is also unimodal. From the change of variable formula, we now that the distribution of $Z$ is given by $\mu_{\mathrm{Y}_{2}}\left(y_{2}\right)=\frac{\mu_{Z}\left(g^{-1}\left(y_{2}\right)\right)}{\left|g^{\prime}\left(g^{-1}\left(y_{2}\right)\right)\right|}$. Since $g^{\prime}(\cdot) \geqslant 0$, this reduces to $\mu_{\mathrm{Y}_{2}}\left(y_{2}\right)=\frac{\mu_{z}\left(g^{-1}\left(y_{2}\right)\right)}{g^{\prime}\left(g^{-1}\left(y_{2}\right)\right)}$. A necessary condition for the existence of a least two modes in $\mu_{\mathrm{Y}_{2}}$ is that there exists $y_{20}$, such that $\mu_{\mathrm{Y}_{2}}$ is decreasing at the left of $y_{20}$ and increasing above it. Therefore, it has to be the case that for any $\delta>0, \varepsilon>0$, with $\delta<\varepsilon$, $g^{\prime}\left(g^{-1}\left(y_{20}-\delta\right)\right) \leqslant g^{\prime}\left(g^{-1}\left(y_{20}\right)\right)$ and $g^{\prime}\left(g^{-1}\left(y_{20}+\delta\right)\right) \leqslant g^{\prime}\left(g^{-1}\left(y_{20}-\delta\right)\right)$. Hence, it has to be the case that $\frac{g^{\prime}\left(g^{-1}\left(y_{20}\right)\right)-g^{\prime}\left(g^{-1}\left(y_{20}-\delta\right)\right)}{\delta} \geqslant 0$ and $\frac{g^{\prime}\left(g^{-1}\left(y_{20}+\delta\right)\right)-g^{\prime}\left(g^{-1}\left(y_{20}-\delta\right)\right)}{\delta} \leqslant 0$. Letting $\delta$ tends toward zero, $g(\cdot)$ should then satisfy $\lim _{y_{2} \uparrow y_{20}} g^{\prime \prime}\left(g^{-1}\left(y_{20}\right)\right) \geqslant 0$ and $\lim _{z \downarrow y_{2}} g^{\prime \prime}\left(g^{-1}\left(y_{20}\right)\right) \leqslant 0$.

Q.E.D

Proof of Proposition 9: Lemma 2 established the functional $g(\cdot)$ that relates $\widehat{\nu}_{i}$ to $\widehat{y}_{i}$. Differentiating $g(\cdot)$, we get

$$
g^{\prime}\left(\widehat{\nu}_{i}\right)= \begin{cases}\frac{\alpha}{1-\alpha} & \text { if } \widehat{\nu}_{i}<\widehat{\underline{\nu}} \\ \frac{\rho \exp \left(\widehat{\nu}_{i}\right)}{1-\rho \exp \left(\widehat{\nu}_{i}\right)} & \text { if } \widehat{\underline{\underline{\nu}}} \leqslant \widehat{\nu}_{i} \leqslant \overline{\widehat{\nu}} \\ \frac{\beta}{1-\beta} & \text { if } \widehat{\nu}_{i}>\widehat{\widehat{\nu}}\end{cases}
$$

with $\rho \equiv \frac{(\alpha \varphi+\beta(1-\varphi))\left(1-\alpha-\frac{1-\beta}{1+x}\right)}{\beta(1-\alpha)-\alpha \frac{1-\beta}{1+x}}$. First, note that, whatever $x \geqslant 0$, for $\underline{\widehat{\nu}} \leqslant \widehat{\nu} \leqslant \overline{\widehat{\nu}}$, we have $g^{\prime \prime}(\widehat{\nu})=$ $\frac{\rho \exp (\widehat{\nu})}{(1-\rho \exp (\widehat{\nu}))^{2}} \geqslant 0$

- When $x=0, \rho=\alpha \varphi+\beta(1-\varphi)$, implying that $g^{\prime}(\underline{\widehat{\underline{\nu}}})=\alpha /(1-\alpha)$ and $g^{\prime}(\overline{\widehat{\nu}})=\beta /(1-\beta)$. Hence, $g^{\prime \prime}(\cdot) \geqslant 0$ over the whole support of $\widehat{\nu}$. From lemma 3 , we now that this rules out bi-modality.

- When $x>0$, we have $g^{\prime}(\underline{\widehat{\underline{\nu}}})=\frac{\alpha}{1-\alpha} \frac{1-\alpha-\frac{1-\beta}{1+x}}{\beta-\alpha}$ and $g^{\prime}(\underline{\widehat{\underline{\nu}}})=\frac{\beta}{1-\beta} \frac{1-\alpha-\frac{1-\beta}{1+x}}{\frac{\beta-\alpha}{1+x}}$. But, since $\beta \in(0,1)$, $\frac{1-\alpha-\frac{1-\beta}{1+x}}{\beta-\alpha}>1$, such that $g^{\prime}(\cdot)$ is increasing for $\nu \in(-\infty, \overline{\widehat{\nu}})$. Conversely, as soon as $x>0$, $\frac{1-\alpha-\frac{1-\beta}{1+x}}{\frac{\beta-\alpha}{1+x}}<1$, which implies that $\lim _{\widehat{\nu} \uparrow \bar{\nu}} g^{\prime}(\widehat{\nu})>\lim _{\widehat{\nu} \downarrow \widehat{\nu}} g^{\prime}(\widehat{\nu})$ Therefore, $g^{\prime}(\cdot)$ is decreasing over some range of values for $\nu$, which creates the possibility of bi-modality.

Q.E.D 